\title{
ON THE THERMODYNAMIC LIMIT FOR HARTREE-FOCK TYPE MODELS
}

\author{
I. CATTO $^{\text {a, }, 1}$, C. LE BRIS ${ }^{\text {b }}$, P.-L. LIONS ${ }^{\text {a }}$ \\ ${ }^{\text {a } C E R E M A D E}$, CNRS UMR 7534, Université Paris-Dauphine, \\ Place de Lattre de Tassigny, 75775 Paris Cedex 16, France \\ ${ }^{\mathrm{b}}$ CERMICS, Ecole Nationale des Ponts et Chaussées, 6 \& 8, avenue Blaise Pascal, \\ Cité Descartes, Champs sur Marne, 77455 Marne-La-Vallée Cedex, France
}

Received 26 April 2000

ABSTRACT. - We continue here our study [10-13] of the thermodynamic limit for various models of Quantum Chemistry, this time focusing on the Hartree-Fock type models. For the reduced Hartree-Fock models, we prove the existence of the thermodynamic limit for the energy per unit volume. We also define a periodic problem associated to the Hartree-Fock model, and prove that it is well-posed.

(C) 2001 L'Association Publications de l'Institut Henri Poincaré. Published by Elsevier B.V. All rights reserved

RÉSUMÉ. - Nous poursuivons dans cet article notre étude systématique [10-13] de la limite thermodynamique de divers modèles issus de la Chimie Quantique Moléculaire, en nous consacrant cette fois aux modèles de type Hartree-Fock. Pour le modèle de Hartree-Fock réduit, nous prouvons que l'énergie par unité de volume a une limite thermodynamique, que nous identifions. Nous définissons également un modèle périodique associé au modèle de HartreeFock, et nous démontrons qu'il est bien posé.

(C) 2001 L'Association Publications de l'Institut Henri Poincaré. Published by Elsevier B.V. All rights reserved

\section{Introduction}

We consider here the thermodynamic limit (or bulk limit) problem for some HartreeFock type models, thereby continuing a long term work that we have begun in [11] with a similar study in the setting of the Thomas-Fermi-von Weizsäcker type models. The results we have obtained in that framework were summarized in [10]. The thermodynamic limit problem for the Hartree type models has been studied in [13] and announced in [12]. Those we shall obtain here have also been announced in [12]. We refer the reader to [11] for a detailed introduction to these issues (see also [13], for a summary).

Briefly speaking, the so-called thermodynamic limit problem consists in examining the behaviour of models for a finite volume of matter when the volume under

\footnotetext{
${ }^{1}$ Work partially supported by the European Union TMR network FMRX-CT 96-0001.
} 
consideration goes to infinity. Since the energy is an extensive thermodynamic quantity, it is expected that the energy per unit volume goes to a finite limit when the volume goes to infinity. It is also expected that the function representing the state of the matter goes also to a limit in some sense. The thermodynamic limit problem we study (that is, for crystals and at zero temperature) may be stated as follows.

We consider a neutral molecule consisting of nuclei of unit charge (atomic units will be adopted in all that follows), and which are located at points $k=\left(k_{1}, k_{2}, k_{3}\right)$ of integral coordinates in $\mathbf{R}^{3}$; each nucleus therefore lies at the center of a cubic unit cell $Q_{k}=\left\{\left(x_{1}, x_{2}, x_{3}\right) \in \mathbf{R}^{3} ;-\frac{1}{2}<x_{i}-k_{i} \leqslant \frac{1}{2}, i=1,2,3\right\}$ (with the convention that $Q_{0}$ will be henceforth denoted by $Q$ ). The set of the positions of these nuclei is then a finite subset $\Lambda$ of the set of all points of integral coordinates that is $\mathbf{Z}^{3} \subset \mathbf{R}^{3}$. The union of all cubic cells whose center is a point of $\Lambda$ is denoted by $\Gamma(\Lambda)$; its volume is denoted by $|\Lambda|$. Since each cell has unit volume and each nucleus is of unit charge, $|\Lambda|$ is also the number of nuclei and the total nuclear charge. It is important to note that, in all that follows, $\Gamma(\Lambda)$ may be viewed as a big box into which the molecule is confined. (This claim may actually be checked rigorously; see Remark 3.2 in Section 3.1 below.) This assumption is standard for statistical physicists, and is compulsory at positive temperatures.

Suppose that for $\Lambda \subset \mathbf{Z}^{3}$ fixed, we have a well-posed model for the ground state of the neutral molecule consisting of $|\Lambda|$ electrons and $|\Lambda|$ nuclei located at the points of $\Lambda$. Let us denote by $I_{\Lambda}$ the ground state energy, and by $\rho_{\Lambda}$ the minimizing electronic density.

Then, the question of the existence of the thermodynamic (or bulk) limit for the model under consideration may be stated as follows:

(i) Does there exist a limit for the energy per unit volume $\frac{1}{|\Lambda|} I_{\Lambda}$ when $|\Lambda|$ goes to infinity?

(ii) Does the minimizing density $\rho_{\Lambda}$ approach a limit $\rho_{\infty}$ (in a sense to be made precise later) when $|\Lambda|$ goes to infinity?

(iii) Does the limit density $\rho_{\infty}$ have the same periodicity as the assumed periodicity of the nuclei?

Let us precise now the scope of this article. We shall not deal here with the physical background of this theoretical problem, and we would rather refer the reader to the textbooks $[6,55]$ and the articles $[27,28]$. The questions we tackle here are indeed close to questions of interest in Solid State Physics, both for theoretical and numerical purposes. For the sake of brevity, we shall not detail here the relationship between our work and Solid State Physics. We only mention some references here, namely [23,42], and also $[2,6,9,40,43,48,49,57]$.

The purpose of our study is twofold: first, we want to check that the molecular model under consideration does have the expected behaviour in the limit of large volumes; second, we wish to set a limit problem that is well-posed mathematically and that can be justified in the most possible rigorous way (in particular with a view to give a sound ground for numerical simulations of the condensed phase).

The models we shall consider here, and which are described in Section 2 below, are issued from Quantum Chemistry, and therefore, they are models that are only valid at zero temperature. From the mathematical viewpoint, the thermodynamic limit problem 
has been extensively studied, in the zero temperature setting as well as in the setting of strictly positive temperatures (see [11] or [13] for a brief historical survey). We shall only mention the ground-breaking work [32] by Lieb and Simon on the thermodynamic limit in the framework of the Thomas-Fermi theory (TF Theory for short). Indeed, this work was at the origin of our own study [11] on the Thomas-Fermi-von Weizsäcker model (TFW model for short), and has largely influenced our work.

In [11], we have proved that the three questions (i)-(ii)-(iii) of the thermodynamic limit problem that we have raised above can be answered positively in the setting of the TFW theory. We find it useful to briefly emphasize the fact that many of the concepts and techniques that we have used in [11] (some of them being inherited from Lieb and Simon, some others being introduced by us in order to treat the TFW case) will be useful here. Taking benefit from the work by Lieb and Simon who had already defined the TF periodic problem, the idea to introduce the periodic TFW problem was straightforward. Our "only" contribution was therefore to prove that the TFW model does converge in the thermodynamic limit to the guessed periodic model.

The Thomas-Fermi type models are derived from the so-called Density Functional Theory. In this framework, the electronic ground state is determined globally by a single function: the electronic density. In the Hartree model [13] and in the Hartree-Fock model that we study now, the $|\Lambda|$ electrons are described by $|\Lambda|$ wave-functions, whose number thus goes to infinity while passing to the thermodynamic limit. The analysis of these models is therefore expected to be much more intricate than in the ThomasFermi case. As a matter of fact, we have not been able to do in the Hartree-Fock setting everything we did in the TFW setting; that is to prove the convergence of the energy per unit volume in the thermodynamic limit. We shall see below that even the guess on the periodic problem is not so obvious for the Hartree-Fock model. Consequently, the mere definition of the limit problems turns out to be a substantial piece of the work. Actually, it is worth emphasizing that the main obstacle we shall encounter comes from the lack of convexity of the Hartree-Fock functional. Indeed, our study of the TFW model [11] (as well as the TF model study by Lieb and Simon [32]) relies in a crucial way upon the convexity of the energy functionals. For the very same lack of convexity, we have not been able in [13] to prove the convergence of the energy per unit volume in the thermodynamic limit for the Hartree model. We have only proved the convergence of the energy per unit volume in the thermodynamic limit for a simplified Hartree model (namely the restricted Hartree model), whose energy functional is convex. However, we have proposed a periodic problem which is likely to be the Hartree model for crystals, and we have proved that this periodic problem is mathematically well-posed.

Similarly, in the Hartree-Fock setting, we shall not be able to prove the convergence of the energy per unit volume in the thermodynamic limit. We shall nevertheless prove the convergence of the energy per unit volume in the thermodynamic limit for a simplified Hartree-Fock model, whose energy functional is convex (namely the reduced HartreeFock model, treated in Section 4).

As far as the Hartree-Fock model is concerned, we shall suggest a periodic problem as a candidate for the thermodynamic limit (see Section 5). We shall prove that this periodic problem is mathematically well-posed. By the way, it is worth emphasizing the fact that the Euler-Lagrange equations that are derived from our periodic HF problem 
are already known in the Quantum Chemistry literature (see, for example, [42]), thereby strengthening our conviction that our model is the correct one.

This paper is organized as follows. The forthcoming Section 2 is devoted to the definition of the general setting we shall work in, and to the detailed presentation of the results we shall establish. Section 3 collects a priori estimates for the reduced Hartree-Fock and the Hartree-Fock models and a detailed description of the so-called Bloch waves (or Floquet) decomposition, which is a well-known tool by Solid State physicists, and which will also play a great role in our study. Section 4 and Section 5 are concerned with the reduced Hartree-Fock and the Hartree-Fock model respectively. The last section of this paper is devoted to various comments and extensions. We shall also describe there some directions of current research.

\section{General setting of the models and main results}

Let us begin this section by defining the molecular models we shall deal with in this article, namely the Hartree-Fock model, and one of its simplified form, the reduced Hartree-Fock model. For the sake of brevity, we shall often abbreviate the names of these models, and write simply the HF and RHF models, respectively.

We recall from the introduction that, for each $\Lambda$, finite subset of $\mathbf{Z}^{3} \subset \mathbf{R}^{3}$, we consider the molecular system consisting of $|\Lambda|$ nuclei of unit charge that are located at the points of $\Lambda$ and of $|\Lambda|$ electrons. We shall henceforth denote by

$$
V_{\Lambda}(x)=\sum_{k \in \Lambda} \frac{1}{|x-k|},
$$

the attraction potential created by the nuclei on the electrons, and by

$$
\frac{1}{2} U_{\Lambda}=\frac{1}{2} \sum_{\substack{m, n \in \Lambda \\ m \neq n}} \frac{1}{|m-n|}
$$

the self-repulsion of the nuclei.

As in [11], we shall also consider the case when the nuclei are not point nuclei but are smeared nuclei. In that case, each Dirac mass located at a point $k$ of $\Lambda$ is replaced by a compactly supported smooth non-negative function of total mass one, typically denoted by $m(\cdot-k)$, and "centered" at that point of $\Lambda$. The regularity of the function $m$ does not play a great role in the sequel, and therefore we shall assume without loss of generality that $m$ is $C^{\infty}$. The potential (2.1) and the repulsion (2.2) are then respectively replaced by

$$
\begin{gathered}
V_{\Lambda}^{m}(x)=\sum_{k \in \Lambda} m \star \frac{1}{|x-k|} \\
\frac{1}{2} U_{\Lambda}^{m}=\frac{1}{2} D\left(\sum_{k \in \Lambda} m(\cdot+k), \sum_{k \in \Lambda} m(\cdot+k)\right)-\frac{1}{2}|\Lambda| D(m, m) .
\end{gathered}
$$


In the above equation, we have as usual denoted by $D(\cdot, \cdot)$ the double integral defined as follows

$$
D(f, f)=\iint_{\mathbf{R}^{3} \times \mathbf{R}^{3}} \frac{f(x) f(y)}{|x-y|} d x d y .
$$

It will be convenient to introduce in this setting the function

$$
m_{\Lambda}=\sum_{k \in \Lambda} m(\cdot-k) .
$$

In this setting of smeared nuclei, we shall also make use of the effective potential $\Phi_{\Lambda}$ defined for each electronic density $\rho_{\Lambda}$ as follows

$$
\Phi_{\Lambda}=\left(m_{\Lambda}-\rho_{\Lambda}\right) \star \frac{1}{|x|} .
$$

We are now in position to introduce the molecular models we shall deal with.

The Hartree-Fock model, which is the most commonly used model in Quantum Molecular Chemistry [41] can be written as follows

$$
I_{\Lambda}^{H F}=\inf \left\{E_{\Lambda}^{H F}(K)+\frac{1}{2} U_{\Lambda} ; K \in \mathcal{K}_{\Lambda}\right\},
$$

where the set of minimization $\mathcal{K}_{\Lambda}$ consists of self-adjoint operators $K$ on $L^{2}\left(\mathbf{R}^{3}\right)$ such that

$$
\mathcal{K}_{\Lambda}=\left\{0 \leqslant K \leqslant \mathbf{1}, \operatorname{Tr} K=|\Lambda|, \operatorname{Tr}\left[\left(-\Delta-V_{\Lambda}\right) K\right]<+\infty\right\},
$$

with 1 denoting the identity on $L^{2}\left(\mathbf{R}^{3}\right)$. The energy functional $E_{\Lambda}^{H F}$ in (2.3) is given by

$$
\begin{aligned}
E_{\Lambda}^{H F}(K)= & \operatorname{Tr}\left[\left(-\Delta-V_{\Lambda}\right) K\right]+\frac{1}{2} \iint_{\mathbf{R}^{3} \times \mathbf{R}^{3}} \frac{\rho(x, x) \rho(y, y)}{|x-y|} d x d y \\
& -\frac{1}{2} \iint_{\mathbf{R}^{3} \times \mathbf{R}^{3}} \frac{|\rho(x, y)|^{2}}{|x-y|} d x d y,
\end{aligned}
$$

with $\rho(\cdot, \cdot)$ denoting the kernel of the Hilbert-Schmidt operator $K$. Let us now define the various quantities that appear in the above definition of the Hartree-Fock model.

The operator $K$ is the so-called (reduced) one-particle density matrix. From the general theory of trace-class operators on $L^{2}\left(\mathbf{R}^{3}\right)$ (see, for example, [44]), any operator $K$ in $\mathcal{K}_{\Lambda}$ admits a complete set of eigenfunctions $\left(\varphi_{n}\right)_{n \geqslant 1}$ in $H^{1}\left(\mathbf{R}^{3}\right)$ associated to the eigenvalues $0 \leqslant \lambda_{n} \leqslant 1$ (counted with multiplicity). Thus we may decompose $K$ along such an eigenbasis of $L^{2}\left(\mathbf{R}^{3}\right)$, in such a way that its Hilbert-Schmidt kernel may be written as

$$
\rho(x, y)=\sum_{n \geqslant 1} \lambda_{n} \varphi_{n}(x) \varphi_{n}^{*}(y) .
$$

Owing to the fact that $K$ is trace-class, the corresponding density is well-defined as a non-negative function in $L^{1}\left(\mathbf{R}^{3}\right)$ through $\rho(x, x)=\sum_{n \geqslant 1} \lambda_{n}\left|\varphi_{n}(x)\right|^{2}$, and $\operatorname{Tr} K=|\Lambda|=$ 
$\int_{\mathbf{R}^{3}} \rho(x, x) d x=\sum_{n \geqslant 1} \lambda_{n}$. Moreover, according to this spectral decomposition of $K$, we may give a sense to

$$
\operatorname{Tr}[-\Delta K]=\sum_{n \geqslant 1} \lambda_{n} \int_{\mathbf{R}^{3}}\left|\nabla \varphi_{n}(x)\right|^{2} d x
$$

while

$$
\operatorname{Tr}\left[V_{\Lambda} K\right]=\sum_{n \geqslant 1} \lambda_{n} \int_{\mathbf{R}^{3}} V_{\Lambda}(x)\left|\varphi_{n}(x)\right|^{2} d x=\int_{\mathbf{R}^{3}} V_{\Lambda}(x) \rho(x, x) d x .
$$

It is a standard fact [30] that this formulation of the Hartree-Fock problem is equivalent to the following one, which might be more familiar to the reader

$$
\begin{aligned}
I_{\Lambda}^{H F}= & \inf \left\{E_{\Lambda}^{H F}\left(\varphi_{1} ; \ldots ; \varphi_{|\Lambda|}\right)+\frac{1}{2} U_{\Lambda} ; \varphi_{i} \in H^{1}\left(\mathbf{R}^{3}\right)\right. \\
& \left.\int_{\mathbf{R}^{3}} \varphi_{i} \varphi_{j}^{*}=\delta_{i, j}, 1 \leqslant i, j \leqslant|\Lambda|\right\} \\
E_{\Lambda}^{H F}\left(\varphi_{1} ; \ldots ; \varphi_{|\Lambda|}\right)= & \sum_{i=1}^{|\Lambda|} \int_{\mathbf{R}^{3}}\left|\nabla \varphi_{i}\right|^{2}-\int_{\mathbf{R}^{3}} V_{\Lambda}(x) \rho(x) d x+\frac{1}{2} \iint_{\mathbf{R}^{3} \times \mathbf{R}^{3}} \frac{\rho(x) \rho(y)}{|x-y|} d x d y \\
& -\frac{1}{2} \iint_{\mathbf{R}^{3} \times \mathbf{R}^{3}} \frac{|\rho(x, y)|^{2}}{|x-y|} d x d y
\end{aligned}
$$

where $\rho(x, y)=\sum_{i=1}^{|\Lambda|} \varphi_{i}(x) \varphi_{i}(y)^{*}, \rho(x)=\rho(x, x)=\sum_{i=1}^{|\Lambda|}\left|\varphi_{i}(x)\right|^{2}$. This equivalence means that every minimizer of (2.3)-(2.5) is a projector with finite rank $|\Lambda|$. In this latter formulation the $\varphi_{i}$ 's are interpreted as the electronic wave-functions. Let us observe that the formulation in terms of density matrices is more intrinsic, and therefore sometimes more convenient to use than the second one. Indeed, for every unitary transform $U$ in $\mathbf{C}^{|\Lambda|}$, and for every orthonormal family $\left(\varphi_{i}\right)_{1 \leqslant i \leqslant|\Lambda|}$ in $H^{1}\left(\mathbf{R}^{3}\right)^{|\Lambda|}$, we obviously have $E_{\Lambda}^{H F}\left(U\left(\varphi_{1} ; \ldots ; \varphi_{|\Lambda|}\right)\right)=E_{\Lambda}^{H F}\left(\varphi_{1} ; \ldots ; \varphi_{|\Lambda|}\right)$, while the density matrices that are respectively associated to $U\left(\varphi_{1} ; \ldots ; \varphi_{|\Lambda|}\right)$ and $\left(\varphi_{1} ; \ldots ; \varphi_{|\Lambda|}\right)$ are the same.

It is of course straightforward to deduce from the point nuclei setting (2.3)-(2.5) the analogous smeared nuclei setting for the HF problem; namely

$$
\begin{aligned}
I_{\Lambda}^{m, H F}= & \inf \left\{\operatorname{Tr}[-\Delta K]+\frac{1}{2} D\left(\rho-m_{\Lambda}, \rho-m_{\Lambda}\right)-\frac{|\Lambda|}{2} D(m, m)\right. \\
& \left.-\frac{1}{2} \iint_{\mathbf{R}^{3} \times \mathbf{R}^{3}} \frac{|\rho(x, y)|^{2}}{|x-y|} d x d y ; K \in \mathcal{K}_{\Lambda}\right\} .
\end{aligned}
$$

We also remark that the equivalence with a standard form of the type (2.7)-(2.8) obviously holds true.

As announced above, we shall also consider in the sequel the following simplified form of the Hartree-Fock model; namely the reduced Hartree-Fock model: 


$$
\begin{aligned}
& I_{\Lambda}^{R H F}=\inf \left\{E_{\Lambda}^{R H F}(K)+\frac{1}{2} U_{\Lambda} ; K \in \mathcal{K}_{\Lambda}\right\}, \\
& E_{\Lambda}^{R H F}(K)=\operatorname{Tr}\left[\left(-\Delta-V_{\Lambda}\right) K\right]+\frac{1}{2} D(\rho, \rho)
\end{aligned}
$$

(where $\mathcal{K}_{\Lambda}$ is still defined by (2.4)), and respectively its analogous smeared nuclei model

$$
\begin{aligned}
I_{\Lambda}^{m, R H F}= & \inf \left\{\operatorname{Tr}[-\Delta K]+\frac{1}{2} D\left(\rho-m_{\Lambda}, \rho-m_{\Lambda}\right)\right. \\
& \left.-\frac{1}{2}|\Lambda| D(m, m) ; K \in \mathcal{K}_{\Lambda}\right\} .
\end{aligned}
$$

In order to turn to the thermodynamic limit problem per se, it is now time to recall the properties of the sequence of sets $\Lambda$ that we shall consider. For the sake of completeness, we recall here the following definition taken from [11] and [32].

DEFINITION 1. - We shall say that a sequence $\left(\Lambda_{i}\right)_{i} \geqslant 1$ of finite subsets of $\mathbf{Z}^{3}$ goes to infinity if the following two conditions hold:

(a) For any finite subset $A \subset \mathbf{Z}^{3}$, there exists $i \in \mathbf{N}$ such that

$$
\forall j \geqslant i, \quad A \subset \Lambda_{j} .
$$

(b) If $\Lambda^{h}$ is the set of points in $\mathbf{R}^{3}$ whose distance to $\partial \Gamma(\Lambda)$ is less than $h(>0)$, then

$$
\lim _{i \rightarrow \infty} \frac{\left|\Lambda_{i}^{h}\right|}{\left|\Lambda_{i}\right|}=0, \quad \forall h>0 .
$$

Condition (b) will be hereafter referred to as the Van Hove condition.

Briefly speaking, a sequence satisfying the Van Hove condition is a sequence for which the 'boundary' is negligible in front of the 'interior'. A sequence of large cubes typically satisfies the conditions of Definition 1 . We shall only consider henceforth so-called Van Hove sequences which are going to infinity in the sense of the above definition. Occasionally, some additional conditions will also be required (see Theorem 2.2). Following the notation of [32,11], we shall write henceforth $\lim _{\Lambda \rightarrow \infty} f(\Lambda)$ instead of $\lim _{i \rightarrow \infty} f\left(\Lambda_{i}\right)$.

Before introducing the Hartree-Fock type periodic models, it is to be noticed that a key-point for their definition is the understanding of laws of interactions between periodically arranged particles. Indeed, owing to the long-range of the Coulomb potential, the electrostatic potential created by the infinite lattice of nuclei cannot be simply $\sum_{k \in \mathbf{Z}^{3}} \frac{1}{|x-k|}$, since this series obviously does not make sense.

We first of all introduce the periodic potential $G$ that is uniquely defined by

$$
-\triangle G=4 \pi\left(-1+\sum_{y \in \mathbf{Z}^{3}} \delta(\cdot-y)\right),
$$

and

$$
\int_{Q} G=0
$$


with $\delta$ being the Dirac measure. Due to our choice of normalization (2.14) for $G$, we also need to define the constant

$$
M=\lim _{x \rightarrow 0}\left[G(x)-\frac{1}{|x|}\right] .
$$

We shall see in the sequel that this periodic potential $G$ (which is also the Green's function of the Laplacian with periodic conditions on the unit cell) is the interaction electrostatic potential created by the periodic distribution of charges of nuclei. We denote

$$
D_{G}(f, f)=\iint_{Q \times Q} f(x) G(x-y) f(y) d x d y .
$$

We also define

$$
f(x)=\frac{1}{|x|}-\int_{Q} \frac{d y}{|x-y|},
$$

and then

$$
f_{\Lambda}(x)=\sum_{k \in \Lambda}\left(\frac{1}{|x-k|}-\int_{Q} \frac{d y}{|x-k-y|}\right) .
$$

It is convenient to rewrite $f_{\Lambda}$ as

$$
f_{\Lambda}=V_{\Lambda}-\chi_{\Gamma(\Lambda)} \star \frac{1}{|x|},
$$

where, more generally, we shall denote by $\chi_{\Omega}$ the characteristic function of the domain $\Omega$. Besides, it is proved in [32], and recalled in [11], that, when $Q$ is a cube,

$$
|f(x)| \leqslant \frac{C}{|x|^{4}}
$$

almost everywhere on $\mathbf{R}^{3}$, for some positive constant $C$, and that $f_{\Lambda}$ converges to the periodic potential $G+d$, for some real constant $d$ that is independent of $\Lambda$, uniformly on compact subsets of $\mathbf{R}^{3} \backslash \mathbf{Z}^{3}$. Moreover, for any compact subset $K$ of $\mathbf{R}^{3}, f_{\Lambda}-$ $\sum_{k \in \Lambda \cap K} \frac{1}{|x-k|}$ converges uniformly on $K$ to $G+d-\sum_{k \in \mathbf{Z}^{3} \cap K} \frac{1}{|x-k|}$ (see [32]). Therefore, we may noteworthy observe that the periodic potential $G$ which was previously defined by (2.13) and (2.14) is also given by

$$
G(x)=\sum_{k \in \mathbf{Z}^{3}}\left(\frac{1}{|x-k|}-\int_{Q} \frac{1}{|x-y-k|} d y\right)-d ;
$$

that is, the sum over the lattice points of the Coulomb potential created by a point charge placed at the center of the unit cube, and which is screened, on each cell, by a uniform background of negative unit charge. This screening effect which is commonly observed 
in thermodynamic limit issues (see $[25-27,32,11,13]$ ) is a consequence of the electrical neutrality of the molecular systems under consideration.

Let us now turn to the periodic problems we want to define. We shall detail in Section 3.2 below the reasons why we need to introduce the following set of operators, which are aimed to become the analogues of the usual density matrices in the periodic case.

DEFINITION 2. - Let $Q^{\star}=\left[-\pi ;+\pi\left[{ }^{3}\right.\right.$, and, for every $\xi$ in $Q^{\star}$,

$$
L_{\xi}^{2}(Q)=\left\{u \in L_{\text {loc }}^{2}\left(\mathbf{R}^{3}\right) ; \mathrm{e}^{-i \xi \cdot x} u \text { is } Q \text {-periodic }\right\} .
$$

We now consider families of operators $K_{\xi}\left(\xi \in Q^{\star}\right)$, which are self-adjoint on $L_{\xi}^{2}(Q)$, and which enjoy the following properties, for almost every $\xi \in Q^{\star}$.

$(\mathrm{H} 2)^{\prime} \quad 0 \leqslant K_{\xi} \leqslant \mathbf{1}$, with $\mathbf{1}$ being the identity on $L_{\xi}^{2}(Q)$;

(H3) the operators $K_{\xi}$ have finite traces, and satisfy $\int_{Q^{\star}} \operatorname{Tr}_{L_{\xi}^{2}(Q)} K_{\xi} \frac{d \xi}{(2 \pi)^{3}}=1$;

(H4) $\operatorname{Tr}_{L_{\xi}^{2}(Q)}\left[-\Delta_{\xi} K_{\xi}\right]<+\infty$ and $\int_{Q^{\star}} \operatorname{Tr}_{L_{\xi}^{2}(Q)}\left[-\Delta_{\xi} K_{\xi}\right] d \xi<+\infty$.

To every such family of operators is associated, in a unique way, a self-adjoint operator $K$ in $L^{2}\left(\mathbf{R}^{3}\right)$, denoted by $K=\int_{Q^{\star}} K_{\xi} \frac{d \xi}{(2 \pi)^{3}}$, such that

(H1) $K$ commutes with the translations of $\mathbf{Z}^{3}$;

(H2) $0 \leqslant K \leqslant 1$.

We denote by $\mathcal{K}$ the set of operators $K=\int_{Q^{\star}} K_{\xi} \frac{d \xi}{(2 \pi)^{3}}$ which satisfy the conditions (H1)(H4) (or equivalently $\left(\mathrm{H}_{2}^{\prime}\right),(\mathrm{H} 3)$ and $\left.(\mathrm{H} 4)\right)$, and we shall call $K$ a periodic density matrix.

In all that follows, we shall denote by $\rho(\xi, \cdot, \cdot)$ the Hilbert-Schmidt kernel of $K_{\xi}$. Owing to the fact that $K_{\xi}$ is a trace-class operator, we may give a sense to $\rho(\xi, x, x)$ as a $Q$-periodic function in $L_{\text {loc }}^{1}\left(\mathbf{R}^{3}\right)$, and to

$$
\rho(x)=\int_{Q^{\star}} \rho(\xi, x, x) \frac{d \xi}{(2 \pi)^{3}} .
$$

Moreover, $\rho(x)$ is also a $Q$-periodic function in $L_{\text {loc }}^{1}\left(\mathbf{R}^{3}\right)$, which will play the role of the electronic density in crystals. Let us emphasize once more the fact that the definitions of the various quantities appearing in the above definitions are made precise in Section 3.2 below, and, more specifically, in Proposition 3.2 therein.

With the help of the above definitions, we are now able to state the periodic minimization problems associated to the above RHF and HF models. First, for the RHF model (2.10)-(2.11), we set:

$$
\begin{gathered}
I_{\text {per }}^{R H F}=\inf \left\{E_{\text {per }}^{R H F}(K) ; K \in \mathcal{K}\right\}, \\
E_{\text {per }}^{\text {RHF }}(K)=\int_{Q^{\star}} \operatorname{Tr}_{L_{\xi}^{2}(Q)}\left[-\Delta K_{\xi}\right] \frac{d \xi}{(2 \pi)^{3}}-\int_{Q} G \rho+\frac{1}{2} D_{G}(\rho, \rho)
\end{gathered}
$$


with $\rho$ being defined by (2.16). The analogous model in the smeared nuclei setting is written

$$
\begin{gathered}
I_{\text {per }}^{m, R H F}=\inf \left\{E_{\text {per }}^{m, R H F}(K) ; K \in \mathcal{K}\right\}, \\
E_{\text {per }}^{m, R H F}(K)=\int_{Q^{\star}} \operatorname{Tr}_{L_{\xi}^{2}(Q)}\left[-\Delta K_{\xi}\right] \frac{d \xi}{(2 \pi)^{3}} \\
+\frac{1}{2} D_{G}(\rho-m, \rho-m)-\frac{1}{2} D_{G}(m, m) .
\end{gathered}
$$

We shall prove in Section 4 the following results.

THEOREM 2.1 (Well-posedness of the RHF periodic problem). - The minimization problem defined by (2.17) and (2.18) (respectively by (2.19) and (2.20)) admits a minimum. In addition, the corresponding minimizing density $\rho$ is unique and, thus, shares the symmetries of the unit cube.

THEOREM 2.2 (Thermodynamic limit for the RHF energy). - We assume that the Van Hove sequence $\Lambda$ satisfies

$$
\lim _{\Lambda \rightarrow \infty} \frac{\left|\Lambda^{h}\right|}{|\Lambda|} \log \left|\Lambda^{h}\right|=0, \quad \forall h>0,
$$

where $\Lambda^{h}$ is defined in Definition 1. In addition, we assume that the unit cell $Q$ of the periodic lattice is a cube.

In the point nuclei case, we have

$$
\lim _{\Lambda \rightarrow \infty} \frac{1}{|\Lambda|} I_{\Lambda}^{R H F}=I_{p e r}^{R H F}+\frac{M}{2},
$$

where the constant $M$ is defined by (2.15). Respectively, in the smeared nuclei case, assuming in addition that $m$ shares the symmetries of the unit cube $Q$, we have

$$
\lim _{\Lambda \rightarrow \infty} \frac{1}{|\Lambda|} I_{\Lambda}^{m, R H F}=I_{\mathrm{per}}^{m, R H F}+\frac{M}{2},
$$

where, this time, the constant $M$ is defined by

$$
M=\iint_{Q \times Q} m(x) m(y)\left[G(x-y)-\frac{1}{|x-y|}\right] d x d y .
$$

Some comments are in order. The reader has remarked that some technical assumptions ( $Q$ is a cube, (2.21), and $m$ has cubic symmetry) have been made in the above theorem. We need these technical assumptions in Section 4, and more precisely in Subsection 4.3 to prove that the upper limit of $\frac{1}{|\Lambda|} I_{\Lambda}^{R H F}$ may be compared from above by $I_{\mathrm{per}}^{R H F}+\frac{M}{2}$. A technical assumption such as (2.21), that is satisfied by all Van Hove sequences except some very pathological ones, already appears in [11]. However, in [11], 
we manage to get rid of all these technical assumptions using another strategy of proof for results like Theorem 2.2; namely the "energy via density" strategy. Here, such a strategy, based upon the convergence of the minimizers, is out of reach. Of course we believe they are not necessary here either. We believe there is room for improvement in our proofs and some other strategy could allow one to do without these assumptions. Unfortunately, we have not been able to do without them so far.

On the contrary, no additional assumption at all is necessary for the other results stated in this work. In particular, the fact that the unit cell is a cube is not important for Theorems 2.1 and 2.3. We shall not repeat this observation in the forthcoming sections, but the reader should keep it in mind. For further comments, we refer the reader to Section 6.

In view of the above theorem, and in view of calculations that will be detailed in Sections 3 and 5 below, we find it natural to introduce the following periodic minimization problem in the Hartree-Fock framework:

$$
\begin{gathered}
I_{\text {per }}^{H F}=\inf \left\{E_{\text {per }}^{H F}(K) ; K \in \mathcal{K}\right\}, \\
E_{\text {per }}^{H F}(K)=\int_{Q^{\star}} \operatorname{Tr}_{L_{\xi}^{2}(Q)}\left[-\Delta K_{\xi}\right] \frac{d \xi}{(2 \pi)^{3}}-\int_{Q} G \rho+\frac{1}{2} D_{G}(\rho, \rho)-\frac{1}{2} E_{\text {exc }}(K),
\end{gathered}
$$

where $\rho(x)$ is still defined by $(2.16)$. With $\rho(\xi ; \cdot, \cdot)$ being the Hilbert-Schmidt of $K_{\xi}$, the Schwarz kernel of $K$ is given by $\rho(x, y)=\int_{Q^{\star}} \rho(\xi ; x, y) \frac{d \xi}{(2 \pi)^{3}}$, and belongs to $L^{2}\left(Q \times \mathbf{R}^{3}\right)$ (at least; see Proposition 3.2 in Section 3.2 below). For some reasons which are made precise later in Section 5, the periodic exchange term $-\frac{1}{2} E_{\text {exc }}(K)$ is then defined by any of the following two equivalent quantities (see Lemma 5.1 in Section 5 below):

$$
\begin{aligned}
E_{e x c}(K) & =\int_{Q} d x \int_{\mathbf{R}^{3}} \frac{|\rho(x, y)|^{2}}{|x-y|} d y \\
& =\iiint_{\left(Q^{\star}\right)^{2} \times Q^{2}} \rho(\xi, x, y) W_{\infty}\left(\xi-\xi^{\prime}, x-y\right) \rho^{*}\left(\xi^{\prime}, x, y\right) d x d y \frac{d \xi d \xi^{\prime}}{(2 \pi)^{6}} .
\end{aligned}
$$

The interaction potential $W_{\infty}$ is defined, for every $\eta$ and $z$ in $\mathbf{R}^{3}$, by

$$
W_{\infty}(\eta, z)=\sum_{k \in \mathbf{Z}^{3}} \frac{\mathrm{e}^{i k \cdot \eta}}{|z+k|} .
$$

The analogous problem in the smeared nuclei case reads

$$
\begin{gathered}
I_{\mathrm{per}}^{m, H F}=\inf \left\{E_{\mathrm{per}}^{m, H F}(K) ; K \in \mathcal{K}\right\}, \\
E_{\mathrm{per}}^{m, H F}(K)=\int_{Q^{\star}} \operatorname{Tr}_{L_{\xi}^{2}(Q)}\left[-\Delta K_{\xi}\right] \frac{d \xi}{(2 \pi)^{3}}+\frac{1}{2} D_{G}(\rho-m, \rho-m)
\end{gathered}
$$




$$
-\frac{1}{2} E_{e x c}(K)-\frac{1}{2} D_{G}(m, m) .
$$

For the Hartree-Fock model, we only have hints which indicate that the limit we suggest above is the correct one. In order to prepare and stimulate future works on the subject, we prove in Section 5 that the periodic Hartree-Fock problem is well-posed, in the following sense.

THEOREM 2.3 (Well-posedness of the HF periodic problem). - The minimization problem defined by (2.23) and (2.24) (respectively by (2.26) and (2.27)) admits a minimum.

Moreover, we establish in Subsection 5.2 the following.

Proposition 2.1. - We assume that the Van Hove sequence $\Lambda$ satisfies (2.21). In addition, we assume that the unit cell $Q$ is a cube, and that there exists a minimizer $K \in \mathcal{K}$ of $I_{\text {per }}^{H F}$ whose density $\rho$ shares the symmetries of the unit cube. Then,

$$
\limsup _{\Lambda \rightarrow \infty} \frac{I_{\Lambda}^{H F}}{|\Lambda|} \leqslant I_{\mathrm{per}}^{H F}+\frac{M}{2}
$$

where $I_{\mathrm{per}}^{H F}$ is defined by (2.23)-(2.24).

Finally, we define the following useful functional transformation which is a particular convex combination, and that we have already used in [11]. It will be again very efficient in the present work in Subsection 4.1, by allowing to take advantage of the convexity of the reduced Hartree-Fock functional, in order to compare from below the lower limit of the energy per unit volume by the corresponding reduced Hartree-Fock periodic model.

DEFINITION 3. - For a given sequence $\Lambda$ and a sequence $\rho_{\Lambda}$ of densities, we call the $\sim-$ transform of $\rho_{\Lambda}$ and denote by $\widetilde{\rho}_{\Lambda}$ the following sequence of functions

$$
\widetilde{\rho}_{\Lambda}=\frac{1}{|\Lambda|} \sum_{k \in \Lambda} \rho_{\Lambda}(\cdot+k)
$$

We shall make use in the sequel of the following notation. If $H$ is a functional space, we denote by $H_{\text {unif }}\left(\mathbf{R}^{3}\right)$ the space

$$
H_{\text {unif }}\left(\mathbf{R}^{3}\right)=\left\{\psi \in \mathcal{D}^{\prime}\left(\mathbf{R}^{3}\right) ; \psi \in H(x+Q) \forall x \in \mathbf{R}^{3}, \sup _{x \in \mathbf{R}^{3}}\|\psi\|_{H(x+Q)}<\infty\right\},
$$

and

$$
H_{\mathrm{per}}^{1}(Q)=\left\{u \in H_{\mathrm{loc}}^{1}\left(\mathbf{R}^{3}\right), u \text { periodic in } x_{i}, i=1,2,3 \text {, of period } 1\right\} .
$$

As announced in the introduction, the sequel of this paper is devoted to the proofs of the above results. 


\section{Preliminaries}

\subsection{A priori estimates for the reduced Hartree-Fock and the Hartree-Fock models}

We begin this section by recalling the existence results of minima for the HartreeFock and the reduced Hartree-Fock models defined in Section 2 through the formula (2.3)-(2.5) and (2.10)-(2.11) respectively. We shall only state the results and make the proofs in the case of point nuclei; stating the analogues in the case of the smeared nuclei brings no additional difficulty and the proofs are even easier in that case (see [11]).

In the Hartree-Fock setting, the existence of a minimizer for neutral molecules for the standard Hartree-Fock model (2.7)-(2.8) has been proved by Lieb and Simon in [33] and by Lions in [37]. Moreover, the equivalence between the standard Hartree-Fock model (2.7)-(2.8) and the Hartree-Fock model stated in terms of density matrices (2.3)-(2.5) (without restricting the minimization to projectors) is due to Lieb [30]. Lieb's proof has been simplified later by Bach [5]. A similar proof by Lions may also be found in [37]. In the reduced Hartree-Fock setting (2.10)-(2.11), the existence of a minimizer $K_{\Lambda} \in \mathcal{K}_{\Lambda}$ for neutral molecules is due to Solovej [52]. It is important to notice that, the energy functional (2.11) is convex with respect to the density matrix. Moreover, thanks to the strict convexity of $\rho \mapsto D(\rho, \rho)$ (this is standard) and of the convexity of the set $\mathcal{K}$, any minimizer $K_{\Lambda}$ of (2.10) leads to the same density which is uniquely defined (it does not depend on the minimizer $K_{\Lambda}$ ) (see [52]). Let us henceforth denote by $\rho_{\Lambda}(x)$ this density.

Let us now begin our study of the thermodynamic limit for these models with getting bounds on the energy per unit volume.

LEMmA 3.1. - Let $\Lambda$ be a Van Hove sequence, then $\frac{1}{|\Lambda|} I_{\Lambda}^{R H F}$ and $\frac{1}{|\Lambda|} I_{\Lambda}^{H F}$ are bounded independently of $\Lambda$.

Proof. - Since the so-called exchange term $-\frac{1}{2} \iint_{\mathbf{R}^{3} \times \mathbf{R}^{3}} \frac{|\rho(x ; y)|^{2}}{|x-y|} d x d y$ appearing in the definition (2.5) of $E_{\Lambda}^{H F}$ is non-positive, it is obvious, from (2.3)-(2.5) and (2.10)-(2.11) that

$$
\frac{1}{|\Lambda|} I_{\Lambda}^{H F} \leqslant \frac{1}{|\Lambda|} I_{\Lambda}^{R H F}
$$

Thus in order to prove the above lemma, we shall first check that $\frac{1}{|\Lambda|} I_{\Lambda}^{R H F}$ is bounded from above, and then, that $\frac{1}{|\Lambda|} I_{\Lambda}^{H F}$ is bounded from below, with bounds that are independent of $\Lambda$.

Let us begin with the bound from above, which is simpler. Let $\varphi \in \mathcal{D}(Q)$ with $\int_{Q} \varphi^{2} d x=1$. For each $k$ in $\Lambda$, we set $\varphi_{k}=\varphi(\cdot-k)$. Then, the trace-class operator $K_{\Lambda}^{0}$ whose Hilbert-Schmidt kernel is defined by $\sum_{k \in \Lambda} \varphi_{k}(x) \varphi_{k}^{*}(y)$ is clearly a test function for $I_{\Lambda}^{R H F}$, with the electronic density being $\rho_{\Lambda}^{0}=\sum_{k \in \Lambda}\left|\varphi_{k}\right|^{2}$. Arguing as in [11], Chapter 3, Section 3.2 for the electrostatic terms, we check successively that

$$
\frac{1}{|\Lambda|} \operatorname{Tr}\left[-\Delta K_{\Lambda}^{0}\right]=\int_{Q}|\nabla \varphi|^{2},
$$

and that 


$$
\lim _{\Lambda \rightarrow \infty} \frac{1}{|\Lambda|}\left(\frac{1}{2} U_{\Lambda}-\int_{\mathbf{R}^{3}} V_{\Lambda} \rho_{\Lambda}+\frac{1}{2} D\left(\rho_{\Lambda}, \rho_{\Lambda}\right)\right)=\frac{M}{2}-\int_{Q} G \rho+\frac{1}{2} D_{G}(\rho, \rho) .
$$

Let us check now the lower bound for the Hartree-Fock energy per unit volume. We first recall that, by virtue of the so-called Lieb-Thirring inequality [35] and its generalization by Lions and Paul [39] to the case of general density matrices), there exists a positive constant $C_{\mathrm{LT}}$, that is independent of $\Lambda$, such that, for any $K$ in $\mathcal{K}_{\Lambda}$ :

$$
C_{\mathrm{LT}} \int_{\mathbf{R}^{3}} \rho^{5 / 3} \leqslant \operatorname{Tr}[-\Delta K]
$$

On the other hand, the Lieb-Oxford inequality [31] gives a lower bound for the exchange term in the following way. There exists a positive constant $C_{\mathrm{LO}}$ that is independent of $\Lambda$, such that, for any $K$ in $\mathcal{K}_{\Lambda}$ with density $\rho$

$$
-C_{\mathrm{LO}} \int_{\mathbf{R}^{3}} \rho^{4 / 3} \leqslant-\iint_{\mathbf{R}^{3} \times \mathbf{R}^{3}} \frac{|\rho(x ; y)|^{2}}{|x-y|} d x d y .
$$

Whence, with the help of (3.1) and (3.2), the HF model may be compared from below by a Thomas-Fermi-Dirac type model (see Lieb [29]), as follows:

$$
E_{\Lambda}^{H F}(K) \geqslant C_{\mathrm{LT}} \int_{\mathbf{R}^{3}} \rho^{5 / 3}-\int_{\mathbf{R}^{3}} V_{\Lambda} \rho+\frac{1}{2} D(\rho, \rho)-C_{\mathrm{LO}} \int_{\mathbf{R}^{3}} \rho^{4 / 3},
$$

for every $K \in \mathcal{K}_{\Lambda}$. The proof of the lower bound for $\frac{1}{|\Lambda|} I_{\Lambda}^{H F}$ is then a consequence of the results obtained by Lieb and Simon [32] and by the authors [11] for the ThomasFermi type models. We first notice that, when $K$ lies in $\mathcal{K}_{\Lambda}$, because of the LiebThirring inequality (3.1), the corresponding electronic density $\rho$ belongs to the set $\left\{\rho \geqslant 0, \rho \in L^{5 / 3}\left(\mathbf{R}^{3}\right), D(\rho, \rho)<+\infty, \int_{\mathbf{R}^{3}} \rho=|\Lambda|\right\}$. In particular, with the help of the Hölder inequality, and since $1 \leqslant \frac{4}{3} \leqslant \frac{5}{3}$, we get

$$
\int_{\mathbf{R}^{3}} \rho^{4 / 3} \leqslant\left(\int_{\mathbf{R}^{3}} \rho^{5 / 3}\right)^{1 / 2}\left(\int_{\mathbf{R}^{3}} \rho\right)^{1 / 2} \leqslant\left(\int_{\mathbf{R}^{3}} \rho^{5 / 3}\right)^{1 / 2}|\Lambda|^{1 / 2} .
$$

In addition, with $f_{\Lambda}=V_{\Lambda}-\chi_{\Gamma(\Lambda)} \star \frac{1}{|x|}$, we recall from [11] that, for every $1 \leqslant p<3$,

$$
\left\|f_{\Lambda}\right\|_{L^{p}\left(\mathbf{R}^{3}\right)} \leqslant C|\Lambda|^{1 / p}
$$

Whence, going back to (3.3),

$$
\begin{aligned}
& E_{\Lambda}^{H F}(K)+\frac{U_{\Lambda}}{2} \\
& \geqslant C_{\mathrm{LT}} \int_{\mathbf{R}^{3}} \rho^{5 / 3}-\int_{\mathbf{R}^{3}} f_{\Lambda} \rho+\frac{1}{2} D\left(\chi_{\Gamma(\Lambda)}-\rho, \chi_{\Gamma(\Lambda)}-\rho\right)
\end{aligned}
$$




$$
\begin{aligned}
& -C_{\mathrm{LO}} \int_{\mathbf{R}^{3}} \rho^{4 / 3}+\frac{U_{\Lambda}}{2}-\frac{1}{2} D\left(\chi_{\Gamma(\Lambda)}, \chi_{\Gamma(\Lambda)}\right) \\
\geqslant & C_{\mathrm{LT}} \int_{\mathbf{R}^{3}} \rho^{5 / 3}-\left\|f_{\Lambda}\right\|_{L^{5 / 2}}\|\rho\|_{L^{5 / 3}}-C_{\mathrm{LO}}\left(\int_{\mathbf{R}^{3}} \rho^{5 / 3}\right)^{1 / 2}|\Lambda|^{1 / 2}-C_{0}|\Lambda| \\
\geqslant & C_{\mathrm{LT}}\|\rho\|_{L^{5 / 3}}^{5 / 3}-|\Lambda|^{2 / 5}\|\rho\|_{L^{5 / 3}}-C_{\mathrm{LO}}\|\rho\|_{L^{5 / 3}}^{5 / 6}|\Lambda|^{1 / 2}-C_{0}|\Lambda|,
\end{aligned}
$$

for every $K \in \mathcal{K}_{\Lambda}$, where, in addition to (3.4) and (3.5) - with $p=\frac{5}{2}$, we have used the following two facts:

$$
\begin{aligned}
& -\int_{\mathbf{R}^{3}} V_{\Lambda} \rho+\frac{1}{2} D(\rho, \rho)+\frac{U_{\Lambda}}{2} \\
& \quad=-\int_{\mathbf{R}^{3}} f_{\Lambda} \rho+\frac{1}{2} D\left(\chi_{\Gamma(\Lambda)}-\rho, \chi_{\Gamma(\Lambda)}-\rho\right)+\frac{U_{\Lambda}}{2}-\frac{1}{2} D\left(\chi_{\Gamma(\Lambda)}, \chi_{\Gamma(\Lambda)}\right),
\end{aligned}
$$

which follows from the definition of $f_{\Lambda}$, and

$$
\left|U_{\Lambda}-D\left(\chi_{\Gamma(\Lambda)}, \chi_{\Gamma(\Lambda)}\right)\right| \leqslant C_{0}|\Lambda|,
$$

for some positive constant $C_{0}$ that is independent of $\Lambda$ [11]. From (3.7) and by setting $X=\frac{\|\rho\|_{L} 5 / 3}{\Lambda^{3 / 5}}$, we finally obtain

$$
\frac{1}{|\Lambda|} I_{\Lambda}^{H F} \geqslant C_{\mathrm{LT}} X^{5 / 3}-X-C_{\mathrm{LO}} X^{5 / 6}-C_{0}
$$

for any $X \geqslant 0$. The function of $X$ which appears in the right-hand side of the above inequality is bounded from below by some constant (independent of $\Lambda$ ) on the set $\{X \geqslant 0\}$. This concludes the proof of the lemma.

From now on, we shall denote by $K_{\Lambda}$ a minimizer of $I_{\Lambda}^{R H F}$ or $I_{\Lambda}^{H F}$ indiscriminately, by $\rho_{\Lambda}(\cdot ; \cdot)$ its kernel, and by $\rho_{\Lambda}=\rho_{\Lambda}(x ; x)$ the corresponding electronic density. As a corollary of Lemma 3.1 and its proof, we have the following:

Proposition 3.1. - There exist positive constants $C$ that are independent of $\Lambda \subset \mathbf{Z}^{3}$ such that the following estimates hold:

$$
\begin{aligned}
& \frac{1}{|\Lambda|} \operatorname{Tr}\left[-\Delta K_{\Lambda}\right] \leqslant C ; \\
& \frac{1}{|\Lambda|} \int_{\mathbf{R}^{3}}\left|\nabla \sqrt{\rho_{\Lambda}}\right|^{2} \leqslant C ; \\
& \frac{1}{|\Lambda|} \int_{\mathbf{R}^{3}} \rho_{\Lambda}^{5 / 3} \leqslant C ; \\
& \frac{1}{|\Lambda|} \int_{\mathbf{R}^{3}} \rho_{\Lambda}^{p} \leqslant C, \quad \text { for every } 1 \leqslant p \leqslant \frac{5}{3} ;
\end{aligned}
$$




$$
\begin{aligned}
& \frac{1}{|\Lambda|} D\left(\chi_{\Gamma(\Lambda)}-\rho_{\Lambda}, \chi_{\Gamma(\Lambda)}-\rho_{\Lambda}\right) \leqslant C ; \quad \text { and } \\
& 0 \leqslant \frac{1}{|\Lambda|} \iint_{\mathbf{R}^{3} \times \mathbf{R}^{3}} \frac{\left|\rho_{\Lambda}(x ; y)\right|^{2}}{|x-y|} d x d y \leqslant C .
\end{aligned}
$$

Remark 3.1.- The bound (3.10f) on the exchange term was postulated in the chemistry literature (see [42]) but, to the best of our knowledge, it was not checked rigorously so far except in the simplified framework of the free electron gas by Friesecke [21]. This bound implies that the exchange term has to be asymptotically of the same order as the volume occupied by the molecule, here $|\Lambda|$. In particular, the exchange term exhibits an asymptotic behaviour in the thermodynamic limit which is completely different from the one of the other electrostatic terms. (Note that each of them behave separately like $|\Lambda|^{5 / 3}$ while their sum globally behaves like $|\Lambda|$ (see [11]).)

Proof of Proposition 3.1. - We argue only in the framework of the Hartree-Fock model, the case of the reduced Hartree-Fock model being even easier to deal with. We first show that (3.10c) holds. Indeed, on the one hand, we know, by Lemma 3.1, that the energy per unit volume $\frac{1}{|\Lambda|} I_{\Lambda}^{H F}$ is bounded from above by some constant independently of $\Lambda$. While, on the other hand, by combining with (3.9) in the special case when $X=\frac{\left\|\rho_{\Lambda}\right\|_{L} 5 / 3}{\Lambda^{3 / 5}}$, and by using Jensen's inequality, we obtain

$$
C \geqslant \frac{1}{|\Lambda|} I_{\Lambda}^{H F} \geqslant C_{1} X^{5 / 3}-C_{2},
$$

where $C, C_{1}$ and $C_{2}$ are positive constants that are independent of $\Lambda$. It is now easy to deduce (3.10c). Hölder's inequality together with (3.10c) yields (3.10d).

The inequality (3.10f) next follows with the help of the Lieb-Oxford inequality (3.2) and (3.10d) - with $p=\frac{4}{3}$. From (3.5) and (3.10c), and using Hölder's inequality, we deduce

$$
\frac{1}{|\Lambda|}\left|\int_{\mathbf{R}^{3}} f_{\Lambda} \rho_{\Lambda}\right| \leqslant C .
$$

We then deduce (3.10e) by comparing (3.10c), (3.10f) and (3.11) with (3.6) and (3.8). Collecting the previous bounds and comparing with the definition (2.5) of $E_{\Lambda}^{H F}\left(K_{\Lambda}\right)$, we check that the last remaining term in the definition of the functional, namely $\operatorname{Tr}\left[-\Delta K_{\Lambda}\right]$, is also of the order of $|\Lambda|$. This gives (3.10a).

We next observe that for every $K$ in $\mathcal{K}_{\Lambda}$, we have

$$
\int_{\mathbf{R}^{3}}|\nabla \sqrt{\rho}|^{2} \leqslant \operatorname{Tr}[-\Delta K] .
$$

Indeed, let $K \in \mathcal{K}_{\Lambda}$ be given, that we decompose along an eigenbasis $\left(\varphi_{n}\right)_{n \geqslant 1} \in H^{1}\left(\mathbf{R}^{3}\right)$ as in Section 2. Thanks to (2.6), we check successively that:

$$
\int_{\mathbf{R}^{3}}|\nabla \sqrt{\rho}|^{2}=\int_{\mathbf{R}^{3}}\left|\nabla\left(\sum_{n \geqslant 1} \lambda_{n}\left|\varphi_{n}\right|^{2}\right)^{1 / 2}\right|^{2}
$$




$$
\begin{aligned}
& =\int_{\mathbf{R}^{3}} \rho^{-1} \sum_{i=1}^{3}\left|\frac{1}{4} \sum_{n \geqslant 1} \lambda_{n}\left(\varphi_{n} \cdot \frac{\partial \varphi_{n}^{*}}{\partial x_{i}}+\varphi_{n}^{*} \cdot \frac{\partial \varphi_{n}}{\partial x_{i}}\right)\right|^{2} \\
& \leqslant \int_{\mathbf{R}^{3}} \rho^{-1} \sum_{i=1}^{3}\left(\sum_{n \geqslant 1} \lambda_{n}\left|\varphi_{n}\right|\left|\frac{\partial \varphi_{n}}{\partial x_{i}}\right|\right)^{2} \\
& \leqslant \int_{\mathbf{R}^{3}} \rho^{-1} \sum_{i=1}^{3}\left(\sum_{n \geqslant 1} \lambda_{n}\left|\varphi_{n}\right|^{2}\right)\left(\sum_{n \geqslant 1} \lambda_{n}\left|\frac{\partial \varphi_{n}}{\partial x_{i}}\right|^{2}\right) \\
& =\int_{\mathbf{R}^{3}} \sum_{n \geqslant 1} \lambda_{n}\left|\nabla \varphi_{n}\right|^{2}=\operatorname{Tr}[-\Delta K]
\end{aligned}
$$

with the help of the Cauchy-Schwarz inequality to obtain (3.14) and with the convention that the quantities inside the integrals in the right-hand sides of (3.13) to (3.14) are zero almost everywhere in the region where $\rho$ itself vanishes. Finally, thanks to (3.12), (3.10b) is a direct consequence of (3.10a). This concludes the proof of the proposition.

From these bounds on the energy per unit volume, we deduce as in $[11,13]$, the following two corollaries.

COROLlaRY 3.1 (Compactness). - For any Van Hove sequence $\Lambda$, we have

$$
\int_{\Gamma(\Lambda)^{c}} \rho_{\Lambda}=\mathrm{o}(|\Lambda|) .
$$

Remark 3.2. - The above corollary says that, asymptotically, $|\Lambda|+\mathrm{o}(|\Lambda|)$ electrons lie in the "big box" $\Gamma(\Lambda)$. With this result together with the fact that the Van Hove condition allows to neglect the surface effects, it turns out that, at zero temperature, any boundary condition for the wave functions or the electronic density on a big box (like Neumann, Dirichlet or periodic boundary conditions) give rise to the same periodic model after passing to the thermodynamic limit. This, of course, may be particularly relevant for numerical computations.

Proof of Corollary 3.1. - This is a direct consequence of (3.10e) (see [11], Section 3 in Chapter 3).

The second corollary makes use of the notion of $\sim$-transform, introduced in [11] and recalled in Definition 3 in Section 2.

COROLLARY 3.2. - For any Van Hove sequence $\Lambda$, the sequence $\sqrt{\widetilde{\rho}_{\Lambda}}$ is bounded in $H_{\text {unif }}^{1}\left(\mathbf{R}^{3}\right)$, independently of $\Lambda$. Moreover,

$$
\lim _{\Lambda \rightarrow \infty} \int_{Q} \widetilde{\rho}_{\Lambda}=1 .
$$

The above bounds on $\widetilde{\rho}_{\Lambda}$, which are easily deduced by a convexity argument from the definition of the $\sim$-transform and from the bounds (3.10b) and (3.10d), will be useful 
while passing to the lower limit on the energy per unit volume for the reduced HartreeFock model in Subsection 4.1.

Let us end this subsection by recalling the following result which asserts that the limit of a sequence of $\sim$-transforms is necessarily periodic. We skip its easy proof, for which all arguments may be found in [11].

Lemma 3.2. - Let $\Lambda$ be a Van Hove sequence in the sense of Definition 1 . Let $f_{\Lambda}$ be a sequence of function such that, either $\left\|f_{\Lambda}\right\|_{L_{\text {unif }}^{p}\left(\mathbf{R}^{3}\right)} \leqslant C$ or $\left\|f_{\Lambda}\right\|_{L^{p}\left(\mathbf{R}^{3}\right)} \leqslant C|\Lambda|^{1 / p}$, for some $p \in[1,+\infty]$ and some constant $C$ that is independent of $\Lambda$. Let us assume that $\tilde{f}_{\Lambda}$ converges to some $\tilde{f}$, almost everywhere on $\mathbf{R}^{3}$, or weakly in $L_{\mathrm{loc}}^{p}-$ when $1 \leqslant p<+\infty$, or in $L^{\infty}-\star$ weak - when $p=+\infty$. Then, $\tilde{f}$ is periodic.

In order to state rigorously the periodic models we shall consider below, we extend in the forthcoming section the classical notion of one-particle density matrix used for molecules to its analogue for crystals. This construction will allow us to set the RHF and HF models for crystals in terms of such "periodic density matrices". These new objects are closely related to the so-called Bloch waves decomposition classically used in Solid State Physics, as we shall see below.

\subsection{Bloch waves decomposition}

Let $Q=\left[-\frac{1}{2} ;+\frac{1}{2}\left[{ }^{3}\right.\right.$ be the unit cube of $\mathbf{R}^{3}$ centered at 0 . We denote by $Q^{\star}=$ $\left[-\pi ;+\pi\left[{ }^{3}\right.\right.$ the unit cell of the dual (or reciprocal) lattice associated to $\mathbf{Z}^{3}$. In full generality, while working with a general periodic lattice (with unit cell still denoted by $Q$ ), $Q^{\star}$ is the so-called Brillouin zone associated to the dual lattice (see, for example, [45, Section XIII-16]).

In the sequel, we shall denote by $K$ a self-adjoint operator in $L^{2}\left(\mathbf{R}^{3}\right)$, which is aimed at being the "periodic density matrix" we are looking for, and that enjoys the following properties:

(H1) $K$ commutes with the translations which leave the periodic lattice $\mathbf{Z}^{3}$ invariant; namely

$$
\forall k \in \mathbf{Z}^{3}, \quad \tau_{k} K=K \tau_{k},
$$

with $\tau_{k}$ being defined by

$$
\tau_{k} \varphi=\varphi(\cdot+k)
$$

for any function $\varphi$ on $\mathbf{R}^{3}$.

(H2) $0 \leqslant K \leqslant \mathbf{1}$, in the sense of self-adjoint operators in $L^{2}\left(\mathbf{R}^{3}\right)$, with $\mathbf{1}$ being the identity operator on $L^{2}\left(\mathbf{R}^{3}\right)$.

Because of (H1), $K$ is not a compact operator. However, taking advantage of this invariance property of $K$, we shall be able to decompose $K$ into a continuous family of compact, and even trace-class, operators, whose spectral decomposition is therefore very simple. It is classical to study the spectral resolution of $K$ as an operator on $L^{2}\left(\mathbf{R}^{3}\right)$ with the help of the so-called Bloch waves decomposition of $K$ which has been introduced by Floquet [20] in the one dimensional case and by Bloch [7] in the general case. We shall explain now the main ingredients of this method following mainly the formalism 
of Reed and Simon [45, Section XIII-16] together with the book by Conca, Planchard and Vanninathan [16]. Among the wide literature which is devoted to the Bloch waves decomposition (and some applications), we refer more specifically the reader to $[2,15$, $17-19,22,24,56]$.

The spirit of this decomposition is the following: We may construct a decomposition of $L^{2}\left(\mathbf{R}^{3}\right)$ according to this invariance by translation. For this purpose, we define $\mathcal{H}=L^{2}\left(Q^{\star} ; L^{2}(Q)\right)$. Then, there is an isometry $U$ between $L^{2}\left(\mathbf{R}^{3}\right)$ and $\mathcal{H}$, the so-called Floquet operator, defined by $U: L^{2}\left(\mathbf{R}^{3}\right) \rightarrow \mathcal{H}$ and

$$
(U \varphi)_{\xi}(x)=\sum_{k \in \mathbf{Z}^{3}} \mathrm{e}^{-i k \cdot \xi} \varphi(x+k), \quad \text { for a.e. } \xi \in Q^{\star}, x \in Q,
$$

for any $\varphi$ in the Schwartz class $\mathcal{S}\left(\mathbf{R}^{3}\right)$. One may check (see [45]) that $U$ is unitary from $L^{2}\left(\mathbf{R}^{3}\right)$ onto $\mathcal{H}$ and that the inverse of $U$ is $U^{*}$ defined, for all $\xi \mapsto g_{\xi}$ in $\mathcal{H}$, by:

$$
\left(U^{*} g\right)(x+k)=\int_{Q^{\star}} \mathrm{e}^{i k \cdot \xi} g_{\xi}(x) \frac{d \xi}{(2 \pi)^{3}},
$$

for all $k \in \mathbf{Z}^{3}$, for a.e. $x \in Q$. We write down explicitly the fact that $U$ is an isometry, using (3.17) and (3.18), and we obtain the following identity

$$
(\varphi ; \psi)_{L^{2}\left(\mathbf{R}^{3}\right)}=\int_{Q^{\star}}\left((U \varphi)_{\xi} ;(U \psi)_{\xi}\right)_{L^{2}(Q)} \frac{d \xi}{(2 \pi)^{3}},
$$

for any functions $\varphi$ and $\psi$ in $L^{2}\left(\mathbf{R}^{3}\right)$, from which we also infer that, in particular:

$$
\|\varphi\|_{L^{2}\left(\mathbf{R}^{3}\right)}^{2}=\int_{Q^{\star}}\left\|(U \varphi)_{\xi}\right\|_{L^{2}(Q)}^{2} \frac{d \xi}{(2 \pi)^{3}} .
$$

Let us make a few comments on the definition (3.17). First of all, the expression appearing in the right-hand side of (3.17) may be seen as a Fourier series expansion with respect to the $\xi$ variable, and whose coefficients lie in $L^{2}(Q)$. Next, it is clear from (3.17) that $\mathrm{e}^{-i x \cdot \xi}(U \varphi)_{\xi}(x)$ is $Q$-periodic. Such functions are often called quasiperiodic functions with quasi-momentum $\xi$. They are known as Bloch waves in the Solid State Physics literature. It is more convenient (and we shall always do it in the following) to look at $(U \varphi)_{\xi}$ as a function lying in $L_{\xi}^{2}(Q)$, with

$$
L_{\xi}^{2}(Q)=\left\{\varphi \in L_{\text {loc }}^{2}\left(\mathbf{R}^{3}\right) / \varphi(x+k)=\mathrm{e}^{i k \cdot \xi} \varphi(x), \forall k \in \mathbf{Z}^{3}, \text { for a.e. } x \in Q\right\},
$$

or, equivalently,

$$
L_{\xi}^{2}(Q)=\left\{\varphi \in L_{\text {loc }}^{2}\left(\mathbf{R}^{3}\right) / \mathrm{e}^{-i \xi \cdot x} \varphi(x) \text { is } Q \text {-periodic }\right\} .
$$

It is clear from the second formulation, that $L_{\xi}^{2}(Q)$, endowed with the usual Hilbert scalar product on $L^{2}(Q)$, is a Hilbert space which is isomorphic to $L^{2}(Q)$. 
With the help of the isomorphism $U$ between $L^{2}\left(\mathbf{R}^{3}\right)$ and $\mathcal{H}$, we now return to the spectral analysis of the operators $K$ satisfying (H1) by following [45].

To the above decomposition of functions in $L^{2}\left(\mathbf{R}^{3}\right)$ into Bloch waves corresponds a so-called direct integral decomposition of $K$ in the sense that there exists a unique function $\xi \mapsto K_{\xi}$ in $L^{\infty}\left(Q^{\star} ; \mathcal{L}\left(L_{\xi}^{2}(Q)\right)\right)$ (in that follows, $\mathcal{L}(X)$ denotes the space of bounded linear operators from $\mathrm{X}$ into itself) such that, for any function $\varphi$ in $L^{2}\left(\mathbf{R}^{3}\right)$ and almost every $\xi$ in $Q^{\star}$ :

$$
(U K \varphi)_{\xi}=K_{\xi}(U \varphi)_{\xi}
$$

Moreover, we also have:

$$
\sup _{\xi \in Q^{\star}}\left\|K_{\xi}\right\|_{\mathcal{L}\left(L_{\xi}^{2}(Q)\right)}=\|K\|_{\mathcal{L}\left(L^{2}\left(\mathbf{R}^{3}\right)\right)} .
$$

And we shall write

$$
K=\int_{Q^{\star}} K_{\xi} \frac{d \xi}{(2 \pi)^{3}}
$$

in order to refer to the decomposition (3.21) of $K$.

The spectral analysis of $K$ now reduces to the spectral analysis of the family of selfadjoint operators $K_{\xi} \in \mathcal{L}\left(L_{\xi}^{2}(Q)\right)$, the parameter $\xi$ varying in $Q^{\star}$. We now enter the details of such a decomposition for a special class of operators satisfying (H1) and (H2) which will appear below in the setting of the periodic reduced Hartree-Fock and the periodic Hartree-Fock models.

From now on, let us denote by $K$ an arbitrary self-adjoint operator satisfying (H1) and (H2). We assume that there exists a kernel representation of $K$ of the form

$$
K \varphi(x)=\int_{\mathbf{R}^{3}} \rho(x ; y) \varphi(y) d y,
$$

say for any function $\varphi$ in $\mathcal{S}\left(\mathbf{R}^{3}\right)$, with $\rho(\cdot ; \cdot) \in L_{\text {loc }}^{2}\left(\mathbf{R}^{3} \times \mathbf{R}^{3}\right)$. Note that (H1) is then equivalently written

$$
\rho(x+k ; y+k)=\rho(x ; y), \quad \text { for every } k \in \mathbf{Z}^{3}, \text { a.e. on } \mathbf{R}^{3} \times \mathbf{R}^{3},
$$

while the self-adjointness of $K$ simply reads

$$
\rho^{*}(x ; y)=\rho(y ; x),
$$

where $z^{*}$ denotes the complex conjugate of $z(\in \mathbf{C})$. We shall now impose further conditions on the kernel $\rho$.

As a consequence of the definition and of the uniqueness of the decomposition (3.23) of $K$, we deduce that each operator $K_{\xi}$ is self-adjoint [45]. Moreover, we have the following

LeMmA 3.3. - Let $K=\int_{Q^{\star}} K_{\xi} \frac{d \xi}{(2 \pi)^{3}}$. Then, $(\mathrm{H} 2)$ is equivalent to: 
$(\mathrm{H} 2)^{\prime} \quad 0 \leqslant K_{\xi} \leqslant 1$ in the sense of self-adjoint operators in $\mathcal{L}\left(L_{\xi}^{2}(Q)\right)$, and for almost every $\xi$ in $Q^{\star}$.

We are searching for operators $K$ for which the $K_{\xi}$ 's are Hilbert-Schmidt, and more specifically, we shall rely upon the following.

LEMmA 3.4. - Let $K=\int_{Q^{\star}} K_{\xi} \frac{d \xi}{(2 \pi)^{3}}$. Then the following two properties are equivalent:

(i)

$$
\rho \in L^{2}\left(Q \times \mathbf{R}^{3}\right) \cap L^{2}\left(\mathbf{R}^{3} \times Q\right) .
$$

(ii) For almost every $\xi$ in $Q^{\star}, K_{\xi}$ is a Hilbert-Schmidt operator with kernel $\rho(\xi ; x ; y)$, and $\rho(\xi ; x ; y) \in L^{2}\left(Q^{\star} ; L^{2}(Q \times Q)\right)$.

Moreover, if $K$ satisfies (i) or (ii), we have

$$
\int_{Q^{\star}} \frac{d \xi}{(2 \pi)^{3}} \iint_{Q \times Q}|\rho(\xi ; x ; y)|^{2} d x d y=\iint_{Q \times \mathbf{R}^{3}}|\rho(x ; y)|^{2} d x d y .
$$

In addition, $\rho$ and $\rho(\xi ; \cdot ; \cdot)$ are related as follows: For almost every $x$ and $y$ in $Q$, and $\xi$ in $Q^{\star}$,

$$
\rho(\xi ; x ; y)=\sum_{k \in \mathbf{Z}^{3}} \mathrm{e}^{-i k \cdot \xi} \rho(x+k ; y)=\sum_{k \in \mathbf{Z}^{3}} \mathrm{e}^{+i k \cdot \xi} \rho(x ; y+k),
$$

hence

$$
\rho(x ; y)=\int_{Q^{\star}} \rho(\xi ; x ; y) \frac{d \xi}{(2 \pi)^{3}} .
$$

Proof of Lemma 3.3. - The proof mimics that of (3.22), which may be found in [45, Section XIII.16 ] (proof of Theorem XIII-83). We shall partially reproduce the argument here for the sake of consistency.

In virtue of (3.22), and since (H2) implies in particular that

$$
\|K\|_{\mathcal{L}\left(L^{2}\left(\mathbf{R}^{3}\right)\right)} \leqslant 1
$$

it just remains to check that $K_{\xi} \geqslant 0$ for almost every $\xi \in Q^{\star}$ as soon as $K \geqslant 0$ (the reverse implication being even easier to prove).

Following [45], we choose a dense subset $\left\{\beta_{k}\right\}_{k} \geqslant 1$ of the unit sphere of $L^{2}(Q)$, and we take an arbitrary function $f \geqslant 0$ in $L^{1}\left(Q^{\star}\right)$. We check now that, for every $k \geqslant 1$,

$$
\int_{Q^{\star}} f(\xi)\left(K_{\xi} \beta_{k} ; \beta_{k}\right)_{L^{2}(Q)} \frac{d \xi}{(2 \pi)^{3}} \geqslant 0 .
$$

Our claim will follow then immediately, since we already know that the $\left(K_{\xi} \beta_{k} ; \beta_{k}\right)$ 's belong to $L^{\infty}\left(Q^{\star}\right)$. Let us first note that $\sqrt{f}$ belongs to $L^{2}\left(Q^{\star}\right)$. Then, if we set $g_{k}=U^{*}\left(\sqrt{f} \beta_{k}\right), g_{k} \in L^{2}\left(\mathbf{R}^{3}\right)$, and by using the definition (3.21) of $K_{\xi}$ together with the definition (3.19) of the scalar product on $\mathcal{H}$, we have 


$$
\begin{aligned}
\int_{Q^{\star}} f(\xi)\left(K_{\xi} \beta_{k} ; \beta_{k}\right)_{L^{2}(Q)} \frac{d \xi}{(2 \pi)^{3}} & =\int_{Q^{\star}}\left(K_{\xi} \sqrt{f(\xi)} \beta_{k} ; \sqrt{f(\xi)} \beta_{k}\right)_{L^{2}(Q)} \frac{d \xi}{(2 \pi)^{3}} \\
& =\int_{Q^{\star}}\left(K_{\xi}\left(U g_{k}\right)_{\xi} ;\left(U g_{k}\right)_{\xi}\right)_{L^{2}(Q)} \frac{d \xi}{(2 \pi)^{3}} \\
& =\int_{Q^{\star}}\left(\left(U K g_{k}\right)_{\xi} ;\left(U g_{k}\right)_{\xi}\right)_{L^{2}(Q)} \frac{d \xi}{(2 \pi)^{3}} \\
& =\left(K g_{k} ; g_{k}\right)_{L^{2}\left(\mathbf{R}^{3}\right)} \geqslant 0,
\end{aligned}
$$

because of $(\mathrm{H} 2)$.

The proof of Lemma 3.4 is based upon the following result that we shall use several times in the sequel:

LEMmA 3.5. - Let $\left(u_{n}(\xi ; \cdot)\right)_{n \geqslant 1}$ be a Hilbert basis of $L_{\xi}^{2}(Q)$ for almost every $\xi$ in $Q^{\star}$, such that $\xi \mapsto u_{n}(\xi ; \cdot)$ (that we shall simply denote by $u_{n}$ in the following) belongs to $\mathcal{H}$. Then, if we set $\varphi_{n}=U^{*} u_{n}$ and, for every $p$ in $\mathbf{Z}^{3}$,

$$
\varphi_{n, p}=\tau_{p} \varphi_{n}=\int_{Q^{\star}} \mathrm{e}^{i p \cdot \xi} u_{n}(\xi ; x) \frac{d \xi}{(2 \pi)^{3}},
$$

the family $\left(\varphi_{n, p}\right)_{n \geqslant 1, p \in \mathbf{Z}^{3}}$ is a Hilbert basis of $L^{2}\left(\mathbf{R}^{3}\right)$.

Remark 3.3. - Before giving the proof of Lemma 3.5, and then the one of Lemma 3.4, let us first note that such a basis exists. Indeed, if $\left(u_{n}\right)$ is a given Hilbert basis of $L^{2}(Q)$ consisting of $Q$-periodic functions (think, for example, of $u_{n}=\mathrm{e}^{2 i \pi n \cdot x}, n \in \mathbf{Z}^{3}$ ), then $u_{n}(\xi ; x)=\mathrm{e}^{i \xi \cdot x} u_{n}(x)$ provides the desired example.

Proof of Lemma 3.5. - Let $\left(u_{n}\right)_{n} \in \mathcal{H}$ and $\left(\varphi_{n, p}\right)_{n, p}$ be defined as in the statement of the above lemma. We first show that the $\left(\varphi_{n, p}\right)_{n \geqslant 1, p \in \mathbf{Z}^{3}}$ 's form an orthonormal family in $L^{2}\left(\mathbf{R}^{3}\right)$. Indeed, let $n, m \geqslant 1$ and let $p, q \in \mathbf{Z}^{3}$. Then, using first the definition (3.19) of the Hilbert scalar product on $\mathcal{H}$, the definition (3.29) of $(\varphi)_{n, p}$, next the orthonormality of $u_{n}(\xi ; \cdot)$ and $u_{m}(\xi ; \cdot)$, and finally the fact that

$$
\int_{Q^{\star}} \mathrm{e}^{i p \cdot \xi} \frac{d \xi}{(2 \pi)^{3}}=\delta_{p, 0},
$$

for every $p \in \mathbf{Z}^{3}$ ( $\delta$,. being the Kronecker symbol), we have

$$
\begin{aligned}
\int_{\mathbf{R}^{3}} \varphi_{n}(x+p) \varphi_{m}^{*}(x+q) d x & =\int_{Q^{\star}}\left(\left(U \varphi_{n, p}\right)_{\xi} ;\left(U \varphi_{m, q}\right)_{\xi}\right)_{L_{\xi}^{2}(Q)} \frac{d \xi}{(2 \pi)^{3}} \\
& =\int_{Q^{\star}} \mathrm{e}^{i(p-q) \cdot \xi} \frac{d \xi}{(2 \pi)^{3}} \int_{Q} u_{n}(\xi ; x) u_{m}^{*}(\xi ; x) d x \\
& =\delta_{n, m} \int_{Q^{\star}} \mathrm{e}^{i(p-q) \cdot \xi} \frac{d \xi}{(2 \pi)^{3}}=\delta_{n, m} \delta_{p, q} .
\end{aligned}
$$


We now check that the Parseval identity holds, thus proving our claim. Indeed, let $\psi \in L^{2}\left(\mathbf{R}^{3}\right)$, then

$$
\begin{aligned}
& \sum_{n \geqslant 1} \sum_{p \in \mathbf{Z}^{3}}\left|\int_{\mathbf{R}^{3}} \psi(x) \varphi_{n}^{*}(x+p) d x\right|^{2} \\
& =\sum_{n \geqslant 1} \sum_{p \in \mathbf{Z}^{3}}\left|\int_{Q^{\star}}\left((U \psi)_{\xi} ;\left(U \varphi_{n, p}\right)_{\xi}\right)_{L_{\xi}^{2}(Q)} \frac{d \xi}{(2 \pi)^{3}}\right|^{2} \\
& =\sum_{n \geqslant 1} \sum_{p \in \mathbf{Z}^{3}} \iint_{Q^{\star} \times Q^{\star}} \mathrm{e}^{i p \cdot\left(\xi-\xi^{\prime}\right)}\left((U \psi)_{\xi} ; u_{n}(\xi ; \cdot)\right)_{L_{\xi}^{2}(Q)} \\
& \quad \times\left((U \psi)_{\xi^{\prime}} ; u_{n}\left(\xi^{\prime} ; \cdot\right)\right)_{L_{\xi^{\prime}}^{2}(Q)}^{*} \frac{d \xi d \xi^{\prime}}{(2 \pi)^{6}} \\
& =\sum_{n \geqslant 1} \int_{Q^{\star}} \frac{d \xi}{(2 \pi)^{3}}\left|\int_{Q}(U \psi)_{\xi}(x) u_{n}^{*}(\xi ; x) d x\right|^{2} \\
& =\int_{Q^{\star}}\left\|(U \psi)_{\xi}\right\|_{L_{\xi}^{2}(Q)}^{2} \frac{d \xi}{(2 \pi)^{3}} \\
& =\|\psi\|_{L^{2}\left(\mathbf{R}^{3}\right)}^{2},
\end{aligned}
$$

where (3.30) follows from the Poisson formula, (3.31) from the Parseval identity, and (3.32) from (3.20).

We may turn now to the

Proof of Lemma 3.4. - Let $\left(u_{n}\right)_{n}$ and $\left(\varphi_{n, p}\right)_{n, p}$ be defined as in Lemma 3.5. With the help of Lemma 3.5, and using first the Parseval identity in $L^{2}\left(\mathbf{R}^{3}\right)$, and then the definition of $\rho$ as the kernel of $K$, we have

$$
\begin{aligned}
\iint_{Q \times \mathbf{R}^{3}}|\rho(x ; y)|^{2} d x d y & =\int_{Q} d x \sum_{n \geqslant 1} \sum_{p \in \mathbf{Z}^{3}}\left|\int_{\mathbf{R}^{3}} \rho(x ; y) \varphi_{n}^{*}(y+p) d y\right|^{2} \\
& =\sum_{n \geqslant 1} \sum_{p \in \mathbf{Z}^{3}}\left\|K \varphi_{n, p}\right\|_{L^{2}(Q)}^{2} \\
& =\sum_{n \geqslant 1}\left\|K \varphi_{n}\right\|_{L^{2}\left(\mathbf{R}^{3}\right)}^{2} \\
& =\sum_{n \geqslant 1} \int_{Q^{\star}}\left\|K_{\xi} u_{n}(\xi ; \cdot)\right\|_{L^{2}(Q)}^{2} \frac{d \xi}{(2 \pi)^{3}}
\end{aligned}
$$

where (3.33) comes from (H1), and (3.34) from the definitions of $K_{\xi}$ and of the scalar product on $\mathcal{H}$. From (3.34), we obtain in particular, that $\sum_{n \geqslant 1}\left\|K_{\xi} u_{n}(\xi ; \cdot)\right\|_{L^{2}(Q)}^{2}$ is finite for almost every $\xi$ in $Q^{\star}$ as soon as (3.25) holds true; this is precisely the definition of $K_{\xi}$ as an Hilbert-Schmidt operator on $L_{\xi}^{2}(Q)$, whose kernel $\rho(\xi ; \cdot ; \cdot)$ belongs to $L^{2}(Q \times Q)$ for almost every $\xi$ in $Q^{\star}$ (see, for example, [44]). Therefore, (ii) holds. If we go back to 
(3.34), we now have

$$
\int_{Q^{\star}} \sum_{n \geqslant 1}\left\|K_{\xi} u_{n}(\xi ; \cdot)\right\|_{L^{2}(Q)}^{2} \frac{d \xi}{(2 \pi)^{3}}=\int_{Q^{\star}} \frac{d \xi}{(2 \pi)^{3}} \iint_{Q \times Q}|\rho(\xi ; x ; y)|^{2} d x d y,
$$

whence (3.26). It is easily seen that the same proof gives in fact the proof of the converse implication (ii) $\Rightarrow$ (i) since at each step of the proof we have argued by equivalence.

Let us now prove (3.27) and (3.28). For almost every $y$ fixed in $Q$, we know from (3.25) that $\rho(\cdot ; y)$ lies in $L^{2}\left(\mathbf{R}^{3}\right)$. Next, (3.27) and (3.28) are two equivalent formulations of the claim that $\rho(\xi ; x ; y)$ is obtained by applying the transformation $U$ in $x$ to $\rho(x ; y)$. By the way, let us note that, because of (3.20), this claim provides another proof of (3.26). Let us check that (3.27) holds. Let $\varphi$ be fixed, say in $\mathcal{S}\left(\mathbf{R}^{3}\right)$, we check successively that

$$
\begin{aligned}
(U K \varphi)_{\xi}(x) & =\sum_{k \in \mathbf{Z}^{3}} \mathrm{e}^{-i k \cdot \xi}(K \varphi)(x+k) \\
& =\sum_{k \in \mathbf{Z}^{3}} \mathrm{e}^{-i k \cdot \xi} \int_{\mathbf{R}^{3}} \rho(x+k ; y) \varphi(y) d y \\
& =\sum_{k \in \mathbf{Z}^{3}} \mathrm{e}^{-i k \cdot \xi} \int_{\mathbf{R}^{3}} \rho^{*}(y ; x+k) \varphi(y) d y \\
& =\sum_{k \in \mathbf{Z}^{3}} \mathrm{e}^{-i k \cdot \xi} \int_{Q^{\star}} \frac{d \xi^{\prime}}{(2 \pi)^{3}} \int_{Q} \sum_{l \in \mathbf{Z}^{3}} \mathrm{e}^{i l \cdot \xi^{\prime}} \rho^{*}(y+l ; x+k)(U \varphi)_{\xi^{\prime}}(y) d y \\
& =\sum_{k \in \mathbf{Z}^{3}} \mathrm{e}^{-i k \cdot \xi} \int_{Q^{\star}} \frac{d \xi^{\prime}}{(2 \pi)^{3}} \int_{Q} \sum_{l \in \mathbf{Z}^{3}} \mathrm{e}^{i l \cdot \xi^{\prime}} \rho(x+k-l ; y)(U \varphi)_{\xi^{\prime}}(y) d y \\
& =\sum_{k \in \mathbf{Z}^{3}} \mathrm{e}^{-i k \cdot \xi} \int_{Q^{\star}} \frac{d \xi^{\prime}}{(2 \pi)^{3}} \int_{Q} \mathrm{e}^{i k \cdot \xi^{\prime}} \sum_{l \in \mathbf{Z}^{3}} \mathrm{e}^{-i l \cdot \xi^{\prime}} \rho(x+l ; y)(U \varphi)_{\xi^{\prime}}(y) d y \\
& =\int_{Q} \sum_{k \in \mathbf{Z}^{3}} \mathrm{e}^{-i k \cdot \xi} \rho(x+k ; y)(U \varphi)_{\xi}(y) d y \\
& =K_{\xi}(U \varphi)_{\xi}(x)=\int_{Q} \rho(\xi ; x ; y)(U \varphi)_{\xi}(y) d y,
\end{aligned}
$$

with (3.19) to deduce (3.35), and the Poisson formula to obtain (3.36). This proves our claim.

We are now ready to state the definition of the admissible "periodic density matrices" we shall work with.

Let $K=\int_{Q^{\star}} K_{\xi} \frac{d \xi}{(2 \pi)^{3}}$ be a self-adjoint operator in $L^{2}\left(\mathbf{R}^{3}\right)$ satisfying (H1). We shall say that $K$ is an admissible periodic density matrix if $K$ satisfies in addition to (H1) the following properties (H2)-(H4):

(H2) $0 \leqslant K \leqslant \mathbf{1}$, 
(H3) for almost every $\xi$ in $Q^{\star}, K_{\xi}$ is a trace-class operator on $L_{\xi}^{2}(Q)$, and

$$
\int_{Q^{\star}} \operatorname{Tr}_{L_{\xi}^{2}(Q)} K_{\xi} \frac{d \xi}{(2 \pi)^{3}}=1
$$

(H4) for almost every $\xi$ in $Q^{\star},-\Delta_{\xi} K_{\xi}$ is a trace-class operator on $L_{\xi}^{2}(Q)$, and

$$
\int_{Q^{\star}} \operatorname{Tr}_{L_{\xi}^{2}(Q)}\left[-\Delta_{\xi} K_{\xi}\right] \frac{d \xi}{(2 \pi)^{3}}<+\infty,
$$

where $-\Delta_{\xi}$ is a notation for the operator $\mathrm{e}^{i \xi \cdot x}\left(-\Delta_{\text {per }}\right) \mathrm{e}^{-i \xi \cdot x}$ acting on $L_{\xi}^{2}(Q)$, and with $-\Delta_{\text {per }}$ denoting the Laplace operator associated to periodic boundary conditions in $Q$. Actually, $-\Delta_{\xi}$ is equivalently defined by $-\Delta=\int_{Q^{\star}}-\Delta_{\xi} \frac{d \xi}{(2 \pi)^{3}}$ according to the definitions (3.21) and (3.23) of the Bloch waves decomposition (see [45]). The set of all admissible periodic density matrices is denoted by $\mathcal{K}$. We collect in the forthcoming Proposition 3.2 various properties of the periodic density matrices in $\mathcal{K}$, that have been proved in the course of this section. But, before that, let us introduce some functional spaces: For every $\xi$ in $Q^{\star}$, and for every $1 \leqslant p \leqslant+\infty$,

$$
L_{\xi}^{p}(Q)=\left\{\varphi \in L_{\mathrm{loc}}^{p}\left(\mathbf{R}^{3}\right) / \tau_{k} \varphi=\mathrm{e}^{i k \cdot \xi} \varphi, \forall k \in \mathbf{Z}^{3}\right\},
$$

and

$$
H_{\xi}^{1}(Q)=\left\{\varphi \in H_{\mathrm{loc}}^{1}\left(\mathbf{R}^{3}\right) / \tau_{k} \varphi=\mathrm{e}^{i k \cdot \xi} \varphi, \forall k \in \mathbf{Z}^{3}\right\} .
$$

Proposition 3.2. - Let $K$ belong to $\mathcal{K}$. Then,

(i) $K$ satisfies the equivalent properties given in Lemma 3.4.

Let $\rho(\xi ; \cdot ; \cdot) \in L^{2}\left(Q^{\star} ; Q \times Q\right)$ denote the Hilbert-Schmidt kernel of $K_{\xi}$.

(ii) For almost every $\xi$ in $Q^{\star}$, there exists a complete set of eigenfunctions $\left(u_{n}(\xi ; \cdot)\right)_{n \geqslant 1}$ of $K_{\xi}$ in $L_{\xi}^{2}(Q)$ corresponding to the non-increasing sequence of eigenvalues $0 \leqslant \lambda_{n}(\xi) \leqslant 1$ (counted with their multiplicity) such that $u_{n}(\xi ; \cdot) \in$ $H_{\xi}^{1}(Q), \xi \mapsto u_{n}(\xi ; \cdot) \in \mathcal{H}$, and such that

$$
\rho(\xi ; x ; y)=\sum_{n \geqslant 1} \lambda_{n}(\xi) u_{n}(\xi ; x) u_{n}^{*}(\xi ; y),
$$

for almost every $\xi$ in $Q^{\star}$.

(iii) For almost every $\xi$ in $Q^{\star}, x \mapsto \rho(\xi ; x ; x)$ is periodic, non-negative, belongs to $L_{\mathrm{unif}}^{1}(Q)$, and may be written

$$
\rho(\xi ; x ; x)=\sum_{n \geqslant 1} \lambda_{n}(\xi)\left|u_{n}(\xi ; x)\right|^{2} \quad \text { a.e. on } Q \text {. }
$$


Hence, $\operatorname{Tr}_{L_{\xi}^{2}(Q)} K_{\xi}=\int_{Q} \rho(\xi ; x ; x) d x$. In addition, we may define $\rho(x ; x)$ by

$$
\rho(x ; x)=\int_{Q^{\star}} \rho(\xi ; x ; x) \frac{d \xi}{(2 \pi)^{3}},
$$

and $\rho(x ; x)$ is a $Q$-periodic, non-negative function in $L_{\text {unif }}^{1}(Q)$. And, we also have

$$
\begin{aligned}
\int_{Q^{\star}} \operatorname{Tr}_{L_{\xi}^{2}(Q)} K_{\xi} \frac{d \xi}{(2 \pi)^{3}} & =\int_{Q^{\star}} \frac{d \xi}{(2 \pi)^{3}} \int_{Q} \rho(\xi ; x ; x) d x \\
& =\int_{Q^{\star}} \sum_{n \geqslant 1} \lambda_{n}(\xi) \frac{d \xi}{(2 \pi)^{3}} \\
& =\int_{Q} \rho(x ; x) d x=1 .
\end{aligned}
$$

(iv) (H4) writes

$$
\int_{Q^{\star}} \operatorname{Tr}_{L_{\xi}^{2}(Q)}\left[-\Delta_{\xi} K_{\xi}\right] \frac{d \xi}{(2 \pi)^{3}}=\int_{Q^{\star}} \sum_{n \geqslant 1} \lambda_{n}(\xi) \frac{d \xi}{(2 \pi)^{3}} \int_{Q}\left|\nabla u_{n}(\xi ; x)\right|^{2} d x<+\infty .
$$

Let us now turn to the thermodynamic limit problem for the RHF model.

\section{The reduced Hartree-Fock model}

This section is devoted to the proof of Theorem 2.2 which has been stated in Section 2 . It is organized as follows. We begin with the hardest part of the work in Subsection 4.1, which consists in verifying that the lower limit of the energy per unit volume may be bounded from below by the periodic RHF model. Subsection 4.2 is then devoted to the proof of Theorem 2.1; that is of the well-posedness of the periodic RHF model. At last, with a minimizer of this periodic model at hand, we are able to check in Subsection 4.3 that the upper limit of the energy per unit volume may be compared from above by the periodic RHF model.

\subsection{Lower limit of the energy per unit volume}

In this section, we bound from below the lower limit of $\frac{I_{\Lambda}^{R H F}}{|\Lambda|}$ as defined in (2.10)(2.11) by the energy of the periodic RHF model (2.17)-(2.18). For the sake of clarity, let us recall here the definitions of these problems:

$$
\begin{gathered}
I_{\Lambda}^{R H F}=\inf \left\{E_{\Lambda}^{R H F}(K)+\frac{1}{2} U_{\Lambda} ; K \in \mathcal{K}_{\Lambda}\right\}, \\
E_{\Lambda}^{R H F}(K)=\operatorname{Tr}\left[\left(-\Delta-V_{\Lambda}\right) K\right]+\frac{1}{2} D(\rho, \rho), \\
I_{\text {per }}^{R H F}=\inf \left\{E_{\text {per }}^{R H F}(K) ; K \in \mathcal{K}\right\},
\end{gathered}
$$




$$
E_{\mathrm{per}}^{R H F}(K)=\int_{Q^{\star}} \operatorname{Tr}_{L_{\xi}^{2}(Q)}\left[-\Delta K_{\xi}\right] \frac{d \xi}{(2 \pi)^{3}}-\int_{Q} G \rho+\frac{1}{2} D_{G}(\rho, \rho),
$$

where the meaning of $\rho=\rho(x ; x)$ is made precise in Proposition 3.2.

Of course, our argument will still apply mutatis mutandis (this is even simpler) to the smeared nuclei case (see (2.12), (2.19), (2.20)). We concentrate ourselves in the sequel on the point nuclei case.

We shall use in a crucial way the fact that the RHF functional $E_{\Lambda}^{R H F}(K)$ is convex with respect to the density matrix $K$, when $K$ belongs to the convex set $\mathcal{K}_{\Lambda}$, for any $\Lambda \subset \mathbf{Z}^{3}$. Indeed, this convexity property will allow us to use the $\sim$-transform trick that we have previously applied to the TFW model in [11]. Let us emphasize the fact that, since we fail in obtaining local bounds on the electronic density in the RHF model (say, for example, $L^{\infty}$ bounds on $\rho_{\Lambda}$ independent of $\Lambda$ ), this is the only method among all the methods presented in [11] which seems to go through to the RHF model.

The sequel of this subsection is devoted to the proof of the following

Proposition 4.1. - Let $\Lambda$ be a Van Hove sequence. Then,

$$
\liminf _{\Lambda \rightarrow \infty} \frac{I_{\Lambda}^{R H F}}{|\Lambda|} \geqslant I_{\mathrm{per}}^{R H F}+\frac{M}{2},
$$

where $I_{\text {per }}^{R H F}$ is defined by (2.17)-(2.18).

Proof. - From now on, we shall denote by $K_{\Lambda}$ a minimizer of $I_{\Lambda}^{R H F}$, by $\rho_{\Lambda}(\cdot ; \cdot)$ its Hilbert-Schmidt kernel and by $\rho_{\Lambda}=\rho_{\Lambda}(x ; x)$ the (unique) corresponding electronic density. In particular, using the fact that $K_{\Lambda}$ admits a complete set of orthonormal eigenfunctions $\left(\psi_{n}\right)_{n} \geqslant 1$ belonging to $H^{1}\left(\mathbf{R}^{3}\right)$, associated to the eigenvalues $0 \leqslant \mu_{n} \leqslant 1$ (counted with their multiplicity), we may write

$$
\rho_{\Lambda}(x ; y)=\sum_{n \geqslant 1} \mu_{n} \psi_{n}(x) \psi_{n}^{*}(y) \quad \text { and } \quad \rho_{\Lambda}(x ; x)=\sum_{n \geqslant 1} \mu_{n}\left|\psi_{n}(x)\right|^{2},
$$

where, here and in all that follows, we have on purpose omitted to mention the dependence of the $\mu_{n}$ 's and of the $\psi_{n}$ 's on $\Lambda$, in order to simplify the notation. Let us recall that

$$
\operatorname{Tr}_{L^{2}\left(\mathbf{R}^{3}\right)} K_{\Lambda}=\sum_{n \geqslant 1} \mu_{n} \int_{\mathbf{R}^{3}}\left|\psi_{n}(x)\right|^{2} d x=|\Lambda|,
$$

and that

$$
0 \leqslant \operatorname{Tr}_{L^{2}\left(\mathbf{R}^{3}\right)}\left[-\Delta K_{\Lambda}\right]=\sum_{n \geqslant 1} \mu_{n} \int_{\mathbf{R}^{3}}\left|\nabla \psi_{n}\right|^{2} d x \leqslant C|\Lambda|,
$$

thanks to (3.10a). Generally speaking, the idea of the strategy detailed below, and which draws its inspiration from [11], is the following. We shall build a particular convex combination from the operators $K_{\Lambda}$, which is more or less a minimizing sequence of $I_{\Lambda}^{R H F}$, but which converges to a periodic density matrix, as $\Lambda$ goes to infinity. Moreover, 
this periodic density matrix will turn to be a minimizer of $I_{\text {per }}^{R H}$. By analogy with the definition of the $\sim$-transform for functions (see Definition 3 in Section 2), we set

$$
\widetilde{K}_{\Lambda}=\frac{1}{|\Lambda|} \sum_{k \in \Lambda} \tau_{k} K_{\Lambda} \tau_{-k}
$$

Then, it is easy to check that $\widetilde{K}_{\Lambda}$ belongs to $\mathcal{K}_{\Lambda}$, that the Hilbert-Schmidt kernel of $\widetilde{K}_{\Lambda}$ is

$$
\widetilde{\rho}_{\Lambda}(x ; y)=\frac{1}{|\Lambda|} \sum_{k \in \Lambda} \rho_{\Lambda}(x+k ; y+k),
$$

while $\widetilde{\rho}_{\Lambda}(x ; x)$ coincides with the usual $\sim$-transform of $\rho_{\Lambda}$ as introduced in [11]. In particular, $\widetilde{K}_{\Lambda}$ also admits a complete set of orthonormal eigenfunctions $\left(\varphi_{n}\right)_{n \geqslant 1}$ belonging to $H^{1}\left(\mathbf{R}^{3}\right)$, associated to the eigenvalues $0 \leqslant \lambda_{n} \leqslant 1$ (counted with their multiplicity), and, therefore, we may write as for $K_{\Lambda}$

$$
\widetilde{\rho}_{\Lambda}(x ; y)=\sum_{n \geqslant 1} \lambda_{n} \varphi_{n}(x) \varphi_{n}^{*}(y) \quad \text { and } \quad \tilde{\rho}_{\Lambda}(x ; x)=\sum_{n \geqslant 1} \lambda_{n}\left|\varphi_{n}(x)\right|^{2} .
$$

Of course, the analogues of (4.1) and (4.2) remain true for $\widetilde{K}_{\Lambda}$.

The proof is organized as follows. We first check that the sequence $\widetilde{K}_{\Lambda}$ defined by (4.3) converges in a sense to be made precise later to some operator $\widetilde{K}$ belonging to $\mathcal{K}$; that is, to a periodic density matrix (Step 1). Moreover, using the two facts that $\sqrt{\widetilde{\rho}_{\Lambda}}$ is bounded in $H_{\text {unif }}^{1}\left(\mathbf{R}^{3}\right)$ (Corollary 3.2) and that its limit is necessarily periodic (Lemma 3.2), we already infer that $\sqrt{\widetilde{\rho}_{\Lambda}}$ converges weakly in $H_{\mathrm{loc}}^{1}\left(\mathbf{R}^{3}\right)$, strongly in $L_{\mathrm{loc}}^{p}\left(\mathbf{R}^{3}\right)$, for every $1 \leqslant p<6$, and almost everywhere on $\mathbf{R}^{3}$ to $\sqrt{\widetilde{\rho}}$, with $\widetilde{\rho}$ being $Q$-periodic, non-negative, and such that $\sqrt{\widetilde{\rho}} \in H_{\text {per }}^{1}(Q)$. The second step, which is much more involved, consists in verifying that the limit $\widetilde{\rho}$ of the density $\widetilde{\rho}_{\Lambda}$ associated to $\widetilde{K}_{\Lambda}$ is also the $Q$-periodic density which is associated to the periodic density matrix $\widetilde{K}$ according to (3.39) in Subsection 3.2, Proposition 3.2 - (iii) (Step 2). Finally, we bound from below the lower limit of the energy per unit volume by $E_{\text {per }}^{R H F}(\widetilde{K})($ Step 3), thereby concluding the proof of Proposition 4.1. Let us already say, at this stage, that thanks to the proof of the upper limit (and thus of the limit), of the energy per unit volume we shall finally deduce that $\widetilde{K}$ is a minimizer of $I_{\mathrm{per}}^{R H F}$, and therefore $\widetilde{\rho}$ is simply $\rho_{\text {per }}$, with $\rho_{\text {per }}$ denoting the unique periodic electronic density that corresponds to any minimizer of $I_{\mathrm{per}}^{R H F}$. In particular, the whole sequence $\sqrt{\widetilde{\rho}_{\Lambda}}$ converges (and not only a subsequence), and its limit is independent of the choice of the Van Hove sequence $\Lambda$.

Step 1.- We first check that the sequence $\widetilde{K}_{\Lambda}$ converges to some operator $\widetilde{K}$ belonging to the set of periodic density matrices $\mathcal{K}$, which is defined through properties (H1)-(H4) in Subsection 3.2 (equivalent to Definition 2 in Section 2).

Since the sequence of operators $\widetilde{K}_{\Lambda}$ is bounded in operators norm, we may extract a subsequence if necessary in such way that $\widetilde{K}_{\Lambda}$ converges to some bounded operator $\widetilde{K}$ in $L^{2}\left(\mathbf{R}^{3}\right)$ for the weak convergence of operators; that is

$$
\left(\widetilde{K}_{\Lambda} \varphi ; \psi\right) \rightarrow(\widetilde{K} \varphi ; \psi) \text { as } \Lambda \rightarrow \infty,
$$


for all $\varphi$ and $\psi$ in $L^{2}\left(\mathbf{R}^{3}\right)$. In particular, $\widetilde{K}$ is a self-adjoint operator in $L^{2}\left(\mathbf{R}^{3}\right)$ and $0 \leqslant \widetilde{K} \leqslant \mathbf{1}$. Therefore, $\widetilde{K}$ enjoys $(\mathrm{H} 2)$.

Let us check now that it also satisfies (H1); that is, let us prove that

(a) $\widetilde{K}$ commutes with the translations which leave $\mathbf{Z}^{3}$ invariant.

Let $\tau$ be such a translation. We fix $\varphi$ and $\psi$ in $L^{2}\left(\mathbf{R}^{3}\right)$, and we intend to prove that

$$
([\widetilde{K}, \tau] \varphi ; \psi)_{L^{2}\left(\mathbf{R}^{3}\right)}=0 .
$$

For this purpose, we make use of a standard argument of [11] which is based upon the fact that the sequence $\Lambda$ is a Van Hove sequence. We just outline this argument here. We have

$$
\begin{aligned}
{\left[\widetilde{K}_{\Lambda}, \tau\right] } & =\frac{1}{|\Lambda|}\left(\sum_{k \in \Lambda} \tau_{-k} K_{\Lambda} \tau_{k} \tau-\sum_{k \in \Lambda} \tau \tau_{-k} K_{\Lambda} \tau_{k}\right) \\
& =\frac{1}{|\Lambda|}\left(\sum_{k \in \tau \Lambda \backslash \Lambda} \tau \tau_{-k} K_{\Lambda} \tau_{k}-\sum_{k \in \Lambda \backslash \tau \Lambda} \tau \tau_{-k} K_{\Lambda} \tau_{k}\right) .
\end{aligned}
$$

Hence

$$
\left|\left(\left[\widetilde{K}_{\Lambda}, \tau\right] \varphi ; \psi\right)_{L^{2}\left(\mathbf{R}^{3}\right)}\right| \leqslant \frac{|\Lambda \backslash \tau \Lambda|}{|\Lambda|}\left\|K_{\Lambda}\right\|\|\varphi\|_{L^{2}\left(\mathbf{R}^{3}\right)}\|\psi\|_{L^{2}\left(\mathbf{R}^{3}\right)}=\mathrm{o}(1)
$$

as $\Lambda$ goes to infinity. As the left-hand side converges to $([\widetilde{K}, \tau] \varphi ; \psi)_{L^{2}\left(\mathbf{R}^{3}\right)}$, this shows the expected invariance.

According to the results and the notation of Section 3.2, we may write $\widetilde{K}=$ $\int_{Q^{\star}} \widetilde{K}_{\xi} \frac{d \xi}{(2 \pi)^{3}}$ with $\widetilde{K}_{\xi}$ being a self-adjoint operator in $L_{\xi}^{2}(Q)$ such that $0 \leqslant \widetilde{K}_{\xi} \leqslant \mathbf{1}$, for almost every $\xi$ in $Q^{\star}$.

Having checked that $(\mathrm{H} 1)$ and $(\mathrm{H} 2)$ are satisfied by $\widetilde{K}$, we next want to verify that $\widetilde{K}$ satisfies (H3). Namely, we now want to check that,

(b) for almost every $\xi$ in $Q^{\star}$, the operator $\widetilde{K}_{\xi}$ has a finite trace on $L_{\xi}^{2}(Q)$, and

$$
\int_{Q^{\star}} \operatorname{Tr}_{L_{\xi}^{2}(Q)} \widetilde{K}_{\xi} \frac{d \xi}{(2 \pi)^{3}}=1 .
$$

According to Lemma 3.5 in Subsection 3.2 , we denote by $\left(u_{n}(\xi ; \cdot)\right)_{n \geqslant 1}$ an arbitrary Hilbert basis of $L_{\xi}^{2}(Q)$ for almost every $\xi$ in $Q^{\star}$, and by $\varphi_{n, p}=\tau_{-p} \varphi_{n}$ the corresponding Hilbert basis of $L^{2}\left(\mathbf{R}^{3}\right)$. (Note that in fact, since $K$ and the $\widetilde{K}_{\xi}$ 's are non-negative, it would be sufficient to make the following argument for one given basis - see [44].) Then, we check successively that:

$$
\begin{aligned}
1 & =\frac{1}{|\Lambda|} \operatorname{Tr}_{L^{2}\left(\mathbf{R}^{3}\right)} K_{\Lambda}=\frac{1}{|\Lambda|} \sum_{n \geqslant 1} \sum_{p \in \mathbf{Z}^{3}}\left(K_{\Lambda} \varphi_{n, p} ; \varphi_{n, p}\right) \\
& \geqslant \frac{1}{|\Lambda|} \sum_{n \geqslant 1} \sum_{p \in \Lambda}\left(K_{\Lambda} \varphi_{n, p} ; \varphi_{n, p}\right)=\sum_{n \geqslant 1} \frac{1}{|\Lambda|} \sum_{p \in \Lambda}\left(\tau_{p} K_{\Lambda} \tau_{-p} \cdot \varphi_{n} ; \varphi_{n}\right) \\
& =\sum_{n \geqslant 1}\left(\widetilde{K}_{\Lambda} \varphi_{n} ; \varphi_{n}\right),
\end{aligned}
$$


the inequality in the above string of equalities coming from the positiveness of $K$. Then, because of the weak convergence of $\widetilde{K}_{\Lambda}$ to $\widetilde{K},\left(\widetilde{K}_{\Lambda} \varphi_{n} ; \varphi_{n}\right)$ converges to $\left(\widetilde{K} \varphi_{n} ; \varphi_{n}\right)$, for every $n \geqslant 1$, as $\Lambda$ goes to infinity. Now, since $\widetilde{K}_{\Lambda} \geqslant 0$, we know that the terms of the series appearing in the right-hand side of (4.4) are all non-negative. We may then appeal to the discrete version of the Fatou lemma to infer that $\sum_{n \geqslant 1}\left(\widetilde{K} \varphi_{n} ; \varphi_{n}\right)<+\infty$, and that

$$
\liminf _{\Lambda \rightarrow \infty} \sum_{n \geqslant 1}\left(\widetilde{K}_{\Lambda} \varphi_{n} ; \varphi_{n}\right) \geqslant \sum_{n \geqslant 1}\left(\widetilde{K} \varphi_{n} ; \varphi_{n}\right) .
$$

Owing to the definitions (3.21) of the notation $\widetilde{K}=\int_{Q^{\star}} \widetilde{K}_{\xi} \frac{d \xi}{(2 \pi)^{3}}$ and (3.19) of the scalar product on $\mathcal{H}$, we have

$$
\begin{aligned}
\sum_{n \geqslant 1}\left(\widetilde{K} \varphi_{n} ; \varphi_{n}\right) & =\sum_{n \geqslant 1} \int_{Q^{\star}}\left(\left(U \widetilde{K} \varphi_{n}\right)_{\xi} ;\left(U \varphi_{n}\right)_{\xi}\right)_{L^{2}(Q)} \frac{d \xi}{(2 \pi)^{3}} \\
& =\sum_{n \geqslant 1} \int_{Q^{\star}}\left(\widetilde{K}_{\xi} u_{n}(\xi ; \cdot) ; u_{n}(\xi ; \cdot)\right)_{L^{2}(Q)} \frac{d \xi}{(2 \pi)^{3}} \\
& =\int_{Q^{\star}} \sum_{n \geqslant 1}\left(\widetilde{K}_{\xi} u_{n}(\xi ; \cdot) ; u_{n}(\xi ; \cdot)\right)_{L^{2}(Q)} \frac{d \xi}{(2 \pi)^{3}} \\
& =\int_{Q^{\star}} \operatorname{Tr}_{L_{\xi}^{2}(Q)} \widetilde{K}_{\xi} \frac{d \xi}{(2 \pi)^{3}} .
\end{aligned}
$$

In particular, collecting with the above string of inequalities, we already know that, for almost every $\xi$ in $Q^{\star}, \widetilde{K}_{\xi}$ is a trace-class operator on $L_{\xi}^{2}(Q)$ and that

$$
\int_{Q^{\star}} \operatorname{Tr}_{L_{\xi}^{2}(Q)} \widetilde{K}_{\xi} \frac{d \xi}{(2 \pi)^{3}} \leqslant 1 .
$$

Moreover, denoting by $\widetilde{\rho}(\xi ; x ; y)$, the Hilbert-Schmidt kernel of $\widetilde{K}_{\xi}$, we may give a sense to $\widetilde{\rho}(\xi ; x ; x)$ as a non-negative periodic function in $L^{1}(Q)$, such that $\operatorname{Tr}_{L_{\xi}^{2}(Q)} \widetilde{K}_{\xi}=$ $\int_{Q} \widetilde{\rho}(\xi ; x ; x) d x$. In addition, thanks to (4.5), we may associate to $\widetilde{K}$, the non-negative $Q$-periodic density $\rho_{\widetilde{K}}$, which is defined by $\rho_{\widetilde{K}}(x ; x)=\int_{Q^{\star}} \widetilde{\rho}(\xi ; x ; x) \frac{d \xi}{(2 \pi)^{3}}$, and which belongs to $L^{1}(Q)$. In order to conclude the proof of (H3), it remains to show that

$$
\int_{Q^{\star}} \operatorname{Tr}_{L_{\xi}^{2}(Q)} \widetilde{K}_{\xi} \frac{d \xi}{(2 \pi)^{3}}=1 .
$$

To prove this claim we shall actually prove in Step 2 below that

$$
\widetilde{\rho}(x)=\rho_{\widetilde{K}}(x ; x)=\int_{Q^{\star}} \widetilde{\rho}(\xi ; x ; x) \frac{d \xi}{(2 \pi)^{3}}
$$


for almost every $x$ in $Q$, where $\widetilde{\rho}$ is the limit of $\widetilde{\rho}_{\Lambda}$. Whence (4.6), thanks to (3.16) in Corollary 3.2.

Admitting (4.7), for a while, we now claim that $\widetilde{K}$ satisfies (H4) i.e. that

(c) for almost every $\xi$ in $Q^{\star},-\Delta_{\xi} \widetilde{K}_{\xi}$ is a trace-class operator on $L_{\xi}^{2}(Q)$, such that

$$
\int_{Q^{\star}} \operatorname{Tr}_{L_{\xi}^{2}(Q)}\left[-\Delta_{\xi} \widetilde{K}_{\xi}\right] \frac{d \xi}{(2 \pi)^{3}}<+\infty,
$$

by proving that

$$
\liminf _{\Lambda \rightarrow \infty} \frac{1}{|\Lambda|} \operatorname{Tr}_{L^{2}\left(\mathbf{R}^{3}\right)}\left[-\Delta K_{\Lambda}\right] \geqslant \int_{Q^{\star}} \operatorname{Tr}_{L_{\xi}^{2}(Q)}\left[-\Delta_{\xi} \widetilde{K}_{\xi}\right] \frac{d \xi}{(2 \pi)^{3}} .
$$

The proof of (4.9) follows the same lines as the proof of (4.5). Indeed, let $\left(u_{n}(\xi ; \cdot)\right)_{n \geqslant 1}$ and $\varphi_{n, p}=\tau_{-p} \varphi_{n}$ be defined as before, with the additional assumptions that $\left(u_{n}(\xi ; \cdot)\right)_{n \geqslant 1}$ $\in H_{\xi}^{1}(Q)$ for almost every $\xi$ in $Q^{\star}$, and that the $\varphi_{n, p}$ 's belong to $H^{1}\left(\mathbf{R}^{3}\right)$. Then, for the same reasons as before, we find

$$
\begin{aligned}
\frac{1}{|\Lambda|} \operatorname{Tr}_{L^{2}\left(\mathbf{R}^{3}\right)}\left[-\Delta K_{\Lambda}\right] & =\frac{1}{|\Lambda|} \sum_{n \geqslant 1} \sum_{p \in \mathbf{Z}^{3}}\left(K_{\Lambda}(-\Delta)^{1 / 2} \varphi_{n, p} ;(-\Delta)^{1 / 2} \varphi_{n, p}\right) \\
& \geqslant \sum_{n \geqslant 1}\left(\widetilde{K}_{\Lambda}(-\Delta)^{1 / 2} \varphi_{n} ;(-\Delta)^{1 / 2} \varphi_{n}\right) .
\end{aligned}
$$

Then, thanks to the weak convergence of $\widetilde{K}_{\Lambda}$ to $\widetilde{K}$, we have, for every $n \geqslant 1$,

$$
\begin{aligned}
& \lim _{\Lambda \rightarrow \infty}\left(\widetilde{K}_{\Lambda}(-\Delta)^{1 / 2} \varphi_{n} ;(-\Delta)^{1 / 2} \varphi_{n}\right) \\
& \quad=\left(\widetilde{K}(-\Delta)^{1 / 2} \varphi_{n} ;(-\Delta)^{1 / 2} \varphi_{n}\right)=\left((-\Delta)^{1 / 2} \widetilde{K}(-\Delta)^{1 / 2} \varphi_{n} ; \varphi_{n}\right) .
\end{aligned}
$$

Moreover, Fatou's lemma still applies since, on the one hand, for every $\Lambda$ and $n$, we get $\left((-\Delta)^{1 / 2} \widetilde{K}_{\Lambda}(-\Delta)^{1 / 2} \varphi_{n} ; \varphi_{n}\right) \geqslant 0$; while, on the other hand, we know from (3.10a) in Proposition 3.1, that $\frac{1}{|\Lambda|} \operatorname{Tr}_{L^{2}\left(\mathbf{R}^{3}\right)}\left[-\Delta K_{\Lambda}\right]$ is bounded independently of $\Lambda$. Therefore, passing to the lower limit as $\Lambda$ goes to infinity in the above inequalities, we get first that

$$
\sum_{n \geqslant 1}\left(-\Delta \widetilde{K} \varphi_{n} ; \varphi_{n}\right)<+\infty
$$

and next, that

$$
\begin{aligned}
& \liminf _{\Lambda \rightarrow \infty} \frac{1}{|\Lambda|} \operatorname{Tr}_{L^{2}\left(\mathbf{R}^{3}\right)}\left[-\Delta K_{\Lambda}\right] \\
& \quad \geqslant \liminf _{\Lambda \rightarrow \infty} \sum_{n \geqslant 1}\left((-\Delta)^{1 / 2} \widetilde{K}_{\Lambda}(-\Delta)^{1 / 2} \varphi_{n} ; \varphi_{n}\right) \\
& \geqslant \sum_{n \geqslant 1}\left(\widetilde{K}(-\Delta)^{1 / 2} \varphi_{n} ;(-\Delta)^{1 / 2} \varphi_{n}\right) \\
& \quad=\int_{Q^{\star}} \sum_{n \geqslant 1}\left(\widetilde{K}_{\xi}\left(-\Delta_{\xi}\right)^{1 / 2} u_{n}(\xi ; \cdot) ;\left(-\Delta_{\xi}\right)^{1 / 2} u_{n}(\xi ; \cdot)\right)_{L^{2}(Q)} \frac{d \xi}{(2 \pi)^{3}}
\end{aligned}
$$




$$
=\int_{Q^{\star}} \operatorname{Tr}_{L_{\xi}^{2}(Q)}\left[-\Delta_{\xi} \widetilde{K}_{\xi}\right] \frac{d \xi}{(2 \pi)^{3}},
$$

since

$$
(-\Delta)^{1 / 2}=\int_{Q^{\star}}\left(-\Delta_{\xi}\right)^{1 / 2} \frac{d \xi}{(2 \pi)^{3}}
$$

Hence (4.8) and (4.9).

We turn now to the proof of (4.7) (thereby proving (4.6)); that is, the fact that

Step 2. - The limit of $\widetilde{\rho}_{\Lambda}, \widetilde{\rho}$, and the periodic density associated to $\widetilde{K}$, which is defined by $\rho_{\widetilde{K}}=\int_{Q^{\star}} \widetilde{\rho}(\xi ; x ; x) \frac{d \xi}{(2 \pi)^{3}}$, coincide.

We are first looking for

(a) A priori estimates on $\widetilde{\rho}_{\Lambda}(x ; y)$.

Since $K_{\Lambda}^{2} \leqslant K_{\Lambda}$, we have

$$
\rho_{\Lambda}(x) \geqslant \int_{\mathbf{R}^{3}} \rho_{\Lambda}(x ; y) \rho_{\Lambda}(y ; x) d y=\int_{\mathbf{R}^{3}}\left|\rho_{\Lambda}(x ; y)\right|^{2} d y
$$

almost everywhere on $\mathbf{R}^{3}$. Thanks to (4.10), we first check that

$$
\widetilde{\rho}_{\Lambda}(x) \geqslant \int_{\mathbf{R}^{3}}\left|\widetilde{\rho}_{\Lambda}(x ; y)\right|^{2} d y,
$$

which in particular implies that

$$
\widetilde{\rho}_{\Lambda}(x ; y) \text { is bounded in } L^{2}\left(Q \times \mathbf{R}^{3}\right) \cap L^{2}\left(\mathbf{R}^{3} \times Q\right)
$$

independently of $\Lambda$, for $\widetilde{\rho}_{\Lambda}(x)$ is bounded in $L^{1}(Q)$. Indeed, because of (4.10), we check successively that

$$
\begin{aligned}
\widetilde{\rho}_{\Lambda}(x) & =\frac{1}{|\Lambda|} \sum_{k \in \Lambda} \rho_{\Lambda}(x+k ; x+k) \\
& \geqslant \frac{1}{|\Lambda|} \sum_{k \in \Lambda} \int_{\mathbf{R}^{3}}\left|\rho_{\Lambda}(x+k ; y)\right|^{2} d y \\
& =\frac{1}{|\Lambda|} \sum_{k \in \Lambda} \int_{\mathbf{R}^{3}}\left|\rho_{\Lambda}(x+k ; y+k)\right|^{2} d y \\
& \geqslant \int_{\mathbf{R}^{3}}\left|\widetilde{\rho}_{\Lambda}(x ; y)\right|^{2} d y,
\end{aligned}
$$

by convexity. We prove now that

$$
\widetilde{\rho}_{\Lambda}(x ; y) \text { is bounded in } L_{x}^{2}\left(Q ; H_{y}^{1}\left(\mathbf{R}^{3}\right)\right) \cap L_{y}^{2}\left(Q ; H_{x}^{1}\left(\mathbf{R}^{3}\right)\right)
$$


independently of $\Lambda$, or, more precisely, that

$$
\int_{Q} d y \int_{\mathbf{R}^{3}}\left|(1-\Delta)_{x}^{1 / 2} \widetilde{\rho}_{\Lambda}(x ; y)\right|^{2} d x \leqslant C,
$$

where $C$ denotes here and below a positive constant that is independent of $\Lambda$. We emphasize the fact that, in the following, we shall use the notation $(1-\Delta)_{x}^{1 / 2} \widetilde{\rho}_{\Lambda}(\cdot, \cdot)$ for the Schwartz kernel of the operator $(1-\Delta)^{1 / 2} \widetilde{K}_{\Lambda}$, which is also $\sum_{n \geqslant 1} \lambda_{n}[(1-$ $\left.\Delta)^{1 / 2} \varphi_{n}\right](\cdot) \varphi_{n}^{*}(\cdot)$. Indeed, we have

$$
\begin{array}{rl}
\int_{Q} & d y \int_{\mathbf{R}^{3}}\left|(1-\Delta)_{x}^{1 / 2} \widetilde{\rho}_{\Lambda}(x ; y)\right|^{2} d x \\
& =\int_{Q} d y \int_{\mathbf{R}^{3}}\left|\frac{1}{|\Lambda|} \sum_{k \in \Lambda}(1-\Delta)_{x}^{1 / 2} \rho_{\Lambda}(x+k ; y+k)\right|^{2} d x \\
& \leqslant \frac{1}{|\Lambda|} \sum_{k \in \Lambda} \int_{Q} d y \iint_{\mathbf{R}^{3}}\left|(1-\Delta)_{x}^{1 / 2} \rho_{\Lambda}(x+k ; y+k)\right|^{2} d x \\
& =\frac{1}{|\Lambda|} \int_{\Gamma(\Lambda)} d y \int_{\mathbf{R}^{3}}\left|(1-\Delta)_{x}^{1 / 2} \rho_{\Lambda}(x ; y)\right|^{2} d x \\
& \leqslant \frac{1}{|\Lambda|} \int_{\mathbf{R}^{3}} d y \int_{\mathbf{R}^{3}}\left|(1-\Delta)_{x}^{1 / 2} \rho_{\Lambda}(x ; y)\right|^{2} d x \\
& =\frac{1}{|\Lambda|} \iint_{\mathbf{R}^{3} \times \mathbf{R}^{3}}\left|\sum_{n \geqslant 1} \mu_{n}\left[(1-\Delta)^{1 / 2} \psi_{n}\right](x) \psi_{n}^{*}(y)\right|^{2} d x d y \\
& =\frac{1}{|\Lambda|} \int_{\mathbf{R}^{3}} \sum_{n \geqslant 1} \mu_{n}^{2}\left|(1-\Delta)^{1 / 2} \psi_{n}(x)\right|^{2} d x \\
& \leqslant \frac{1}{|\Lambda|} \operatorname{Tr}_{L^{2}\left(\mathbf{R}^{3}\right)}\left[(1-\Delta) K_{\Lambda}\right] .
\end{array}
$$

The first inequality is deduced by a convexity argument, and the last one comes from (4.1), (4.2) and the fact that $0 \leqslant \mu_{n} \leqslant 1$. Finally, we conclude with the help of (3.10a) in Proposition 3.1. An easy consequence of (4.13) is that $\widetilde{\rho}_{\Lambda}(x ; y)$ is bounded in $H_{\text {unif }}^{1}\left(\mathbf{R}^{3} \times \mathbf{R}^{3}\right)$. Thus, up to a subsequence, it converges to some function $\bar{\rho}(x ; y)$ weakly in $H_{\text {loc }}^{1}\left(\mathbf{R}^{3} \times \mathbf{R}^{3}\right)$, strongly in $L_{\text {loc }}^{p}\left(\mathbf{R}^{3} \times \mathbf{R}^{3}\right)$, for every $1 \leqslant p<3$ (by the Rellich theorem for bounded domains of $\mathbf{R}^{6}$ ), and almost everywhere on $\mathbf{R}^{6}$. Actually, because of the weak convergence of $\widetilde{K}_{\Lambda}$ to $\widetilde{K}, \bar{\rho}(x ; y)$ is nothing but $\widetilde{\rho}(x ; y)$, the Schwartz kernel of $\widetilde{K}$. Note that, in particular, we obtain from (4.12) (respectively (4.14)) that, up to a further subsequence, $\widetilde{\rho}_{\Lambda}(x ; y)$ (respectively $\left.(1-\Delta)_{x}^{1 / 2} \widetilde{\rho}_{\Lambda}(x ; y)\right)$ converges to $\widetilde{\rho}(x ; y)$ 
(respectively $\left.(1-\Delta)_{x}^{1 / 2} \widetilde{\rho}(x ; y)\right)$ weakly in $L^{2}\left(Q_{x} \times \mathbf{R}_{y}^{3}\right)$. Therefore, we have

$$
\int_{Q} d x \int_{\mathbf{R}^{3}}|\widetilde{\rho}(x ; y)|^{2} d y<+\infty,
$$

and

$$
\int_{Q} d y \int_{\mathbf{R}^{3}}\left|(1-\Delta)_{x}^{1 / 2} \widetilde{\rho}(x ; y)\right|^{2} d x<+\infty .
$$

The first bound provides another proof of the fact that $\widetilde{K}_{\xi}$ is Hilbert-Schmidt on $L_{\xi}^{2}(Q)$ for almost every $\xi \in Q^{\star}$, with kernel $\widetilde{\rho}(\xi ; x ; y)$, and that $\widetilde{\rho}(x ; y)=\int_{Q^{\star}} \widetilde{\rho}(\xi ; x ; y) \frac{d \xi}{(2 \pi)^{3}}$, by using Lemma 3.4.

Our next step now consists in showing that

$$
(1-\Delta)_{x}^{1 / 2} \widetilde{\rho}_{\Lambda}(x+y, y) \text { is bounded in } L_{x}^{\infty}\left(\mathbf{R}^{3} ; L_{y}^{1}(Q)\right) .
$$

This claim will be a consequence of the following two bounds. First, since $\widetilde{\rho}_{\Lambda}$ is bounded in $L_{\text {unif }}^{1}\left(\mathbf{R}^{3}\right)$, we clearly have

$$
\sup _{t \in \mathbf{R}^{3}} \sum_{n \geqslant 1} \lambda_{n} \int_{t+Q}\left|\varphi_{n}(x)\right|^{2} d x \leqslant C .
$$

Next, we now prove that

$$
\sup _{t \in \mathbf{R}^{3}} \sum_{n \geqslant 1} \lambda_{n} \int_{t+Q}\left|(1-\Delta)^{1 / 2} \varphi_{n}(x)\right|^{2} \leqslant C .
$$

Indeed, using the fact that the self-adjoint operators $(1-\Delta)^{1 / 2} K_{\Lambda}(1-\Delta)^{1 / 2}$ and $(1-\Delta)^{1 / 2} \widetilde{K}_{\Lambda}(1-\Delta)^{1 / 2}$ are positive and trace-class with Hilbert-Schmidt kernels being respectively defined by $\sum_{n \geqslant 1} \mu_{n}\left[(1-\Delta)^{1 / 2} \psi_{n}\right](x)\left[(1-\Delta)^{1 / 2} \psi_{n}\right]^{*}(y)$ and $\sum_{n \geqslant 1} \lambda_{n}[(1-$ $\left.\Delta)^{1 / 2} \varphi_{n}\right](x)\left[(1-\Delta)^{1 / 2} \varphi_{n}\right]^{*}(y)$, we may observe that

$$
\sum_{n \geqslant 1} \lambda_{n}\left|(1-\Delta)^{1 / 2} \varphi_{n}(x)\right|^{2}=\frac{1}{|\Lambda|} \sum_{n \geqslant 1} \mu_{n}\left|(1-\Delta)^{1 / 2} \psi_{n}(x+k)\right|^{2},
$$

almost everywhere on $\mathbf{R}^{3}$, thanks to the definition of $\widetilde{K}_{\Lambda}$. Therefore, for every $t$ in $\mathbf{R}^{3}$,

$$
\begin{aligned}
& \sum_{n \geqslant 1} \lambda_{n} \int_{t+Q}\left|(1-\Delta)^{1 / 2} \varphi_{n}(x)\right|^{2} d x \\
& \quad=\frac{1}{|\Lambda|} \sum_{n \geqslant 1} \mu_{n} \int_{t+\Gamma(\Lambda)}\left|(1-\Delta)^{1 / 2} \psi_{n}(x)\right|^{2} d x \\
& \quad \leqslant \frac{1}{|\Lambda|} \sum_{n \geqslant 1} \mu_{n} \int_{\mathbf{R}^{3}}\left|(1-\Delta)^{1 / 2} \psi_{n}(x)\right|^{2} d x
\end{aligned}
$$




$$
\leqslant C \frac{1}{|\Lambda|} \operatorname{Tr}\left[(1-\Delta) K_{\Lambda}\right] \leqslant C
$$

because of (3.10a). Let us now prove (4.15). For almost every $x$ in $\mathbf{R}^{3}$, and by a repeated use of the Cauchy-Schwarz inequality, we obtain

$$
\begin{aligned}
& \int_{Q}\left|(1-\Delta)_{x}^{1 / 2} \widetilde{\rho}_{\Lambda}(x+y, y)\right| d y \\
& =\int_{Q}\left|\sum_{n \geqslant 1} \lambda_{n}\left[(1-\Delta)^{1 / 2} \varphi_{n}\right](x+y) \varphi_{n}^{*}(y)\right| d y \\
& \leqslant \int_{Q}\left(\sum_{n \geqslant 1} \lambda_{n}\left|(1-\Delta)^{1 / 2} \varphi_{n}(x+y)\right|^{2}\right)^{1 / 2}\left(\sum_{n \geqslant 1} \lambda_{n}\left|\varphi_{n}(y)\right|^{2}\right)^{1 / 2} d y \\
& \leqslant\left(\int_{Q} \sum_{n \geqslant 1} \lambda_{n}\left|(1-\Delta)^{1 / 2} \varphi_{n}(x+y)\right|^{2} d y\right)^{1 / 2}\left(\int_{Q} \sum_{n \geqslant 1} \lambda_{n}\left|\varphi_{n}(y)\right|^{2} d y\right)^{1 / 2},
\end{aligned}
$$

and (4.15) follows, thanks to (4.16) and (4.17).

At this stage, we observe that in particular (4.14) yields

$$
(1-\Delta)_{x}^{1 / 2} \widetilde{\rho}_{\Lambda}(x+y, y) \text { is bounded in } L_{x}^{2}\left(\mathbf{R}^{3} ; L_{y}^{2}(Q)\right) .
$$

Therefore, by a standard interpolation argument,

$$
(1-\Delta)_{x}^{1 / 2} \widetilde{\rho}_{\Lambda}(x+y, y) \text { is bounded in } L_{x}^{p}\left(\mathbf{R}^{3} ; L_{y}^{p^{\prime}}(Q)\right),
$$

with $\frac{1}{p}+\frac{1}{p^{\prime}}=1$, and for every $2 \leqslant p \leqslant+\infty$. In particular, extracting a further subsequence if necessary, we may assume that $(1-\Delta)_{x}^{1 / 2} \widetilde{\rho}_{\Lambda}(x+y, y)$ converges to $(1-\Delta)_{x}^{1 / 2} \widetilde{\rho}(x+y, y)$ weakly in $L_{x}^{p}\left(\mathbf{R}^{3} ; L_{y}^{p^{\prime}}(Q)\right)$, for every $2 \leqslant p<+\infty$.

(b) Proof of (4.7).

Let $\theta$ be a continuous real-valued function, which is compactly supported in the unit cube $Q$, and let us denote $\theta_{\Lambda}=\sum_{k \in \Lambda} \theta(\cdot-k)$. (Note that $\theta_{\Lambda}$ has compact support in $\Gamma(\Lambda)$.) Then, on the one hand, we have

$$
\frac{1}{|\Lambda|} \operatorname{Tr}_{L^{2}\left(\mathbf{R}^{3}\right)}\left[K_{\Lambda} \theta_{\Lambda}\right]=\frac{1}{|\Lambda|} \int_{\mathbf{R}^{3}} \rho_{\Lambda} \theta_{\Lambda}=\int_{Q} \widetilde{\rho}_{\Lambda} \theta,
$$

and, thus,

$$
\lim _{\Lambda \rightarrow \infty} \frac{1}{|\Lambda|} \operatorname{Tr}_{L^{2}\left(\mathbf{R}^{3}\right)}\left[K_{\Lambda} \theta_{\Lambda}\right]=\int_{Q} \tilde{\rho}(x) \theta(x) d x .
$$

On the other hand, we now prove that

$$
\lim _{\Lambda \rightarrow \infty} \frac{1}{|\Lambda|} \operatorname{Tr}_{L^{2}\left(\mathbf{R}^{3}\right)}\left[K_{\Lambda} \theta_{\Lambda}\right]=\int_{Q} d x \int_{Q^{\star}} \widetilde{\rho}(\xi ; x ; x) \theta(x) \frac{d \xi}{(2 \pi)^{3}} .
$$


Since $\theta$ is arbitrary, comparing (4.20) and (4.21) completes the proof of (4.7). We now prove (4.21), and we begin with recalling that the self-adjoint operator $(1-\Delta)^{1 / 2} K_{\Lambda}(1-$ $\Delta)^{1 / 2}$ is Hilbert-Schmidt (and even trace-class) on $L^{2}\left(\mathbf{R}^{3}\right)$, with kernel $(1-\Delta)_{x}^{1 / 2}(1-$ $\Delta)_{y}^{1 / 2} \rho_{\Lambda}(x, y)=\sum_{n \geqslant 1} \mu_{n}\left[(1-\Delta)^{1 / 2} \psi_{n}\right](x)\left[(1-\Delta)^{1 / 2} \psi_{n}\right]^{*}(y)$, for $\operatorname{Tr}_{L^{2}\left(\mathbf{R}^{3}\right)}[(1-$ $\left.\Delta) K_{\Lambda}\right]$

$<+\infty$. Besides, due to the fact that $\theta_{\Lambda}$ is a continuous function with compact support, it is a known fact that the self-adjoint operator $(1-\Delta)^{-1 / 2} \theta_{\Lambda}(1-\Delta)^{-1 / 2}$ is HilbertSchmidt on $L^{2}\left(\mathbf{R}^{3}\right)$, whose kernel is denoted by $\Theta_{\Lambda}(x, y)$. With these observations, we write

$$
\begin{aligned}
& \operatorname{Tr}_{L^{2}\left(\mathbf{R}^{3}\right)}\left[K_{\Lambda} \theta_{\Lambda}\right] \\
& \quad=\operatorname{Tr}_{L^{2}\left(\mathbf{R}^{3}\right)}\left[(1-\Delta)^{1 / 2} K_{\Lambda}(1-\Delta)^{1 / 2}(1-\Delta)^{-1 / 2} \theta_{\Lambda}(1-\Delta)^{-1 / 2}\right] \\
& \quad=\iint_{\mathbf{R}^{3} \times \mathbf{R}^{3}}\left[(1-\Delta)_{x}^{1 / 2}(1-\Delta)_{y}^{1 / 2} \rho_{\Lambda}(x, y)\right] \Theta_{\Lambda}(x, y) d x d y \\
& \quad=\sum_{n \geqslant 1} \mu_{n} \int_{\mathbf{R}^{3}} d x\left[(1-\Delta)^{1 / 2} \psi_{n}\right](x)\left(\int_{\mathbf{R}^{3}} \Theta_{\Lambda}(x, y)\left[(1-\Delta)^{1 / 2} \psi_{n}\right]^{*}(y) d y\right)
\end{aligned}
$$

thanks to Fubini's theorem. We shall now use the explicit form of the Hilbert-Schmidt kernel of $(1-\Delta)^{-1 / 2} \theta_{\Lambda}(1-\Delta)^{-1 / 2}$. Indeed, recalling that, by definition of $(1-\Delta)^{-1 / 2}$, $\mathcal{F}\left[(1-\Delta)^{-1 / 2} \varphi\right](x)=\left(1+|x|^{2}\right)^{-1 / 2} \mathcal{F} \varphi(x)$ (where $\mathcal{F}$ denotes the Fourier transform), it is not difficult to verify that, if $\varphi$ is, say, in the Schwartz class, we have

$$
(1-\Delta)^{-1 / 2} \varphi=G_{1} \star \varphi,
$$

where $G_{1}$ is a function in $L^{1}\left(\mathbf{R}^{3}\right)$ whose Fourier transform is simply the function $\left(1+|x|^{2}\right)^{-1 / 2}\left(\in L^{p}\left(\mathbf{R}^{3}\right)\right.$, for every $\left.3<p \leqslant+\infty\right)$. The function $G_{1}$ is a special kind of Bessel functions, and, from [53], for example, we know that $G_{1}$ is a non-negative radially symmetric function, such that

$$
G_{1}(x) \leqslant C_{1} \exp \left(-\frac{1}{2}|x|\right), \quad \text { for }|x| \text { large enough, }
$$

and

$$
G_{1}(x)=C_{2} \frac{1}{|x|^{2}}+\mathrm{o}\left(\frac{1}{|x|^{2}}\right), \quad \text { as }|x| \rightarrow 0,
$$

for some positive constants $C_{1}$ and $C_{2}$. In particular, $G_{1}$ actually belongs to $L^{3 / 2, \infty}\left(\mathbf{R}^{3}\right) \cap$ $L^{p}\left(\mathbf{R}^{3}\right)$, for every $1 \leqslant p<\frac{3}{2}$.

Therefore, for any continuous function $\vartheta$ with, say, a compact support, the Schwartz kernel of $(1-\Delta)^{-1 / 2} \vartheta(1-\Delta)^{-1 / 2}$ may be written as $\int_{\mathbf{R}^{3}} G_{1}(x-z) \vartheta(z) G_{1}(z-y) d z$.

Then, by definition of $\Theta_{\Lambda}$,

$$
\begin{aligned}
& \int_{\mathbf{R}^{3}} \Theta_{\Lambda}(x, y)\left[(1-\Delta)^{1 / 2} \psi_{n}\right]^{*}(y) d y \\
& \quad=(1-\Delta)^{-1 / 2} \theta_{\Lambda}(1-\Delta)^{-1 / 2}\left(\left[(1-\Delta)^{1 / 2} \psi_{n}\right]^{*}\right)(x)
\end{aligned}
$$




$$
=(1-\Delta)^{-1 / 2}\left(\theta_{\Lambda} \psi_{n}^{*}\right)=G_{1} \star\left(\theta_{\Lambda} \psi_{n}^{*}\right) .
$$

Thus, using Fubini's theorem again,

$$
\begin{aligned}
\sum_{n \geqslant 1} & \mu_{n} \int_{\mathbf{R}^{3}} d x\left[(1-\Delta)^{1 / 2} \psi_{n}\right](x)\left(\int_{\mathbf{R}^{3}} \Theta_{\Lambda}(x, y)\left[(1-\Delta)^{1 / 2} \psi_{n}\right]^{*}(y) d y\right) \\
= & \iint_{\mathbf{R}^{3} \times \mathbf{R}^{3}} G_{1}(x-y) \theta_{\Lambda}(y)\left(\sum_{n \geqslant 1} \mu_{n}\left[(1-\Delta)^{1 / 2} \psi_{n}\right](x) \psi_{n}^{*}(y)\right) d x d y \\
& =\iint_{\mathbf{R}^{3} \times \mathbf{R}^{3}} G_{1}(x-y) \theta_{\Lambda}(y)(1-\Delta)_{x}^{1 / 2} \rho_{\Lambda}(x, y) d x d y .
\end{aligned}
$$

Therefore, we deduce, comparing with (4.22),

$$
\begin{aligned}
& \frac{1}{|\Lambda|} \operatorname{Tr}_{L^{2}\left(\mathbf{R}^{3}\right)}\left[K_{\Lambda} \theta_{\Lambda}\right] \\
& =\frac{1}{|\Lambda|} \iint_{\mathbf{R}^{3} \times \mathbf{R}^{3}} G_{1}(x-y) \theta_{\Lambda}(y)(1-\Delta)_{x}^{1 / 2} \rho_{\Lambda}(x, y) d x d y \\
& =\frac{1}{|\Lambda|} \int_{\Gamma(\Lambda)} d y \int_{\mathbf{R}^{3}} G_{1}(x-y) \theta_{\Lambda}(y)(1-\Delta)_{x}^{1 / 2} \rho_{\Lambda}(x, y) d x \\
& =\frac{1}{|\Lambda|} \sum_{k \in \Lambda} \int_{Q} d y \int_{\mathbf{R}^{3}} G_{1}(x-k-y) \theta(y)(1-\Delta)_{x}^{1 / 2} \rho_{\Lambda}(x, y+k) d x \\
& =\frac{1}{|\Lambda|} \sum_{k \in \Lambda} \int_{Q} d y \int_{\mathbf{R}^{3}} G_{1}(x-y) \theta(y)(1-\Delta)_{x}^{1 / 2} \rho_{\Lambda}(x+k, y+k) d x \\
& =\int_{Q} d y \int_{\mathbf{R}^{3}} G_{1}(x-y) \theta(y)(1-\Delta)_{x}^{1 / 2} \widetilde{\rho}_{\Lambda}(x, y) d x \\
& =\int_{Q} d y \int_{\mathbf{R}^{3}} G_{1}(x) \theta(y)(1-\Delta)_{x}^{1 / 2} \widetilde{\rho}_{\Lambda}(x+y, y) d x .
\end{aligned}
$$

We now make use of the two facts that $(1-\Delta)_{x}^{1 / 2} \widetilde{\rho}_{\Lambda}(x+y, y)$ converges to $(1-$ $\Delta)_{x}^{1 / 2} \widetilde{\rho}(x+y, y)$, weakly in, say $L_{x}^{4}\left(\mathbf{R}^{3} ; L_{y}^{4 / 3}(Q)\right)$, and that $G(x) \theta(y)$ belongs to $L_{x}^{4 / 3}\left(\mathbf{R}^{3}\right) \times L_{y}^{4}(Q)$. Therefore,

$$
\begin{aligned}
\lim _{\Lambda \rightarrow \infty} \frac{1}{|\Lambda|} \operatorname{Tr}_{L^{2}\left(\mathbf{R}^{3}\right)}\left[K_{\Lambda} \theta_{\Lambda}\right] & =\int_{Q} d y \int_{\mathbf{R}^{3}} G_{1}(x) \theta(y)(1-\Delta)_{x}^{1 / 2} \widetilde{\rho}(x+y, y) d x \\
& =\int_{Q} d y \int_{\mathbf{R}^{3}} G_{1}(x-y) \theta(y)(1-\Delta)_{x}^{1 / 2} \widetilde{\rho}(x, y) d x \\
& =\int_{Q} d x \int_{Q^{\star}} \theta(x) \widetilde{\rho}(\xi ; x ; x) \frac{d \xi}{(2 \pi)^{3}} .
\end{aligned}
$$


Indeed, we first observe that the self-adjoint operator $(1-\Delta)^{-1 / 2}$ is bounded on $L^{2}\left(\mathbf{R}^{3}\right)$ and commutes with the group of the translations of $\mathbf{Z}^{3}$. Therefore, we may apply the abstract Bloch wave decomposition (that is explained in Section 3.2) to $(1-\Delta)^{-1 / 2}$, and we have $(1-\Delta)^{-1 / 2}=\int_{Q^{\star}}(1-\Delta)_{\xi}^{-1 / 2} \frac{d \xi}{(2 \pi)^{3}}$, with the operator $(1-\Delta)_{\xi}^{-1 / 2}$ being defined by

$$
\left(U(1-\Delta)^{-1 / 2} \varphi\right)_{\xi}(x)=(1-\Delta)_{\xi}^{-1 / 2}(U \varphi)_{\xi}=\int_{Q} G_{\xi}(x-y)(U \varphi)_{\xi}(y) d y
$$

thanks to (4.23), for every $\varphi$ in $L^{2}\left(\mathbf{R}^{3}\right)$, and for almost every $x$ in $Q$ and $\xi$ in $Q^{\star}$, and where, according to (4.23),

$$
G_{\xi}(t)=\sum_{k \in \mathbf{Z}^{3}} \mathrm{e}^{-i k \cdot \xi} G_{1}(t+k) .
$$

(Note that, from the definition of $(-\Delta)_{\xi},(1-\Delta)_{\xi}^{-1 / 2}=\left(1-\Delta_{\xi}\right)^{-1 / 2}$.) Finally, we conclude as follows

$$
\begin{array}{rl}
\int_{Q} & d y \int_{\mathbf{R}^{3}} G_{1}(x-y) \theta(y)(1-\Delta)_{x}^{1 / 2} \widetilde{\rho}(x, y) d x \\
= & \int_{Q} \theta(y) d y \int_{Q} d x \int_{Q^{\star}} G_{\xi}(x-y)\left[\left(1-\Delta_{\xi}\right)_{x}^{1 / 2} \widetilde{\rho}\right](\xi ; x ; y) \frac{d \xi}{(2 \pi)^{3}} \\
= & \int_{Q} \theta(y) d y \sum_{n \geqslant 1} \iint_{Q \times Q^{\star}} G_{\xi}(x-y) \lambda_{n}(\xi)\left[(1-\Delta)_{\xi}^{1 / 2} u_{n}(\xi, x)\right] u_{n}^{*}(\xi, y) \frac{d \xi d x}{(2 \pi)^{3}} \\
= & \sum_{n \geqslant 1} \iint_{Q \times Q^{\star}} \theta(y) \lambda_{n}(\xi) u_{n}^{*}(\xi, y)\left(\int_{Q} G_{\xi}(x-y)(1-\Delta)_{\xi}^{1 / 2} u_{n}(\xi, x) d x\right) \frac{d \xi d y}{(2 \pi)^{3}} \\
= & \int_{Q} \theta(y) d y \sum_{n \geqslant 1} \int_{Q^{\star}} \lambda_{n}(\xi)\left|u_{n}(\xi, y)\right|^{2} \frac{d \xi}{(2 \pi)^{3}} \\
= & \int_{Q} \theta(y) d y \int_{Q^{\star}} \widetilde{\rho}(\xi ; y ; y) \frac{d \xi}{(2 \pi)^{3}} .
\end{array}
$$

This completes the proof of (4.21).

The kinetic energy term being settled with (4.9), we now turn to

Step 3. - Lower limit of the sum of the electrostatic terms. Conclusion.

We shall first rewrite the sum of electrostatic terms in a more convenient (and equivalent) manner which has been introduced in [11, Section 3.4]. We shall only sketch the argument and refer the reader to [11] for more details. The electrostatic terms in the energy are the following

$$
\begin{gathered}
-\operatorname{Tr}_{L^{2}\left(\mathbf{R}^{3}\right)}\left[V_{\Lambda} K_{\Lambda}\right]+\frac{1}{2} D\left(\rho_{\Lambda}, \rho_{\Lambda}\right)+\frac{1}{2} U_{\Lambda} \\
=-\int_{\mathbf{R}^{3}} V_{\Lambda} \rho_{\Lambda}+\frac{1}{2} D\left(\rho_{\Lambda}, \rho_{\Lambda}\right)+\frac{1}{2} U_{\Lambda} .
\end{gathered}
$$


Denoting by $f_{\Lambda}(x)=\sum_{k \in \Lambda}\left(\frac{1}{|x-k|}-\int_{Q} \frac{d y}{|x-k-y|}\right)=V_{\Lambda}-\chi_{\Gamma(\Lambda)} \star \frac{1}{|x|}$, we rewrite the sum of the electrostatic terms as follows

$$
\begin{aligned}
\frac{1}{2} \sum_{z \in \Lambda} \lim _{\substack{x \rightarrow z \\
x \neq z}}\left[f_{\Lambda}(x)-\frac{1}{|x-z|}\right]+\frac{1}{2} \int_{\Gamma(\Lambda)} f_{\Lambda} \\
\quad-\int_{\Gamma(\Lambda)} f_{\Lambda} \rho_{\Lambda}+\frac{1}{2} D\left(\chi_{\Gamma(\Lambda)}-\rho_{\Lambda}, \chi_{\Gamma(\Lambda)}-\rho_{\Lambda}\right) .
\end{aligned}
$$

We next remark (see [11, Chapter 2]) that we have

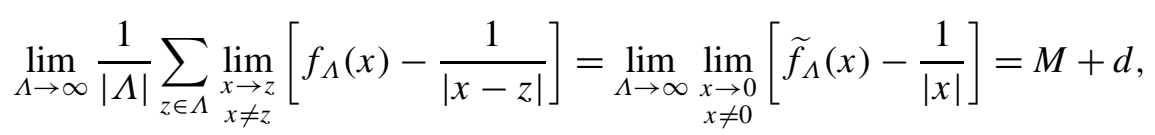

where $d$ is some constant which is related to our choice of normalization for the potential $G$ and which is defined in [11]. In addition, we have

$$
\lim _{\Lambda \rightarrow \infty} \frac{1}{|\Lambda|} \int_{\Gamma(\Lambda)} f_{\Lambda}=\lim _{\Lambda \rightarrow \infty} \int_{Q} \tilde{f}_{\Lambda}=\int_{Q}(G+d)=d,
$$

and

$$
\lim _{\Lambda \rightarrow \infty} \frac{1}{|\Lambda|} \int_{\mathbf{R}^{3}} f_{\Lambda} \rho_{\Lambda}=d+\int_{Q} G(y) \widetilde{\rho}(y) d y .
$$

Therefore, if we prove that

$$
\liminf _{\Lambda \rightarrow \infty} \frac{1}{|\Lambda|} D\left(\chi_{\Gamma(\Lambda)}-\rho_{\Lambda}, \chi_{\Gamma(\Lambda)}-\rho_{\Lambda}\right) \geqslant D_{G}(\widetilde{\rho}, \widetilde{\rho}),
$$

we shall easily deduce from (4.26), (4.27), (4.28), and the formulation (4.25) of the sum of the electrostatic terms (4.24) that

$$
\begin{gathered}
\liminf _{\Lambda \rightarrow \infty} \frac{1}{|\Lambda|}\left(-\operatorname{Tr}\left[V_{\Lambda} K_{\Lambda}\right]+\frac{1}{2} D\left(\rho_{\Lambda}, \rho_{\Lambda}\right)+\frac{1}{2} U_{\Lambda}\right) \\
\geqslant-\int_{Q} G(y) \widetilde{\rho}(y) d y+\frac{1}{2} D_{G}(\widetilde{\rho}, \widetilde{\rho})+\frac{M}{2} .
\end{gathered}
$$

We now prove (4.29). Let us define $g_{\Lambda}=\left(\chi_{\Gamma(\Lambda)}-\rho_{\Lambda}\right) \star \frac{1}{|x|}$. It is a standard fact that

$$
\frac{1}{|\Lambda|} D\left(\chi_{\Gamma(\Lambda)}-\rho_{\Lambda}, \chi_{\Gamma(\Lambda)}-\rho_{\Lambda}\right)=\frac{1}{|\Lambda|} \int_{\mathbf{R}^{3}}\left|\nabla g_{\Lambda}\right|^{2} \geqslant \int_{Q}\left|\nabla \widetilde{g}_{\Lambda}\right|^{2} .
$$

From the bound (3.10e) in Proposition 3.1, we deduce

$$
\int_{Q}\left|\nabla \widetilde{g}_{\Lambda}\right|^{2} \leqslant C
$$


where $\widetilde{g}_{\Lambda}$ is defined, as usual, by $\widetilde{g}_{\Lambda}=\frac{1}{|\Lambda|} \sum_{k \in \Lambda} g_{\Lambda}(\cdot+k)$. Thus, $\nabla \widetilde{g}_{\Lambda}$ is bounded in $L_{\text {unif }}^{2}\left(\mathbf{R}^{3}\right)^{3}$ independently of $\Lambda$. Therefore, extracting a subsequence if necessary, we may assume that there exists $\widetilde{h}$ in $L_{\text {unif }}^{2}\left(\mathbf{R}^{3}\right)^{3}$, such that $\nabla \widetilde{g}_{\Lambda}$ converges to $\widetilde{h} \in L_{\text {unif }}^{2}\left(\mathbf{R}^{3}\right)^{3}$, for the weak convergence in $L_{\text {loc }}^{2}\left(\mathbf{R}^{3}\right)^{3}$, and curl $\widetilde{h}=0$, in the sense of distributions. Moreover, from Poincaré's theorem, there exists $\widetilde{g}$ in $\mathcal{D}^{\prime}\left(\mathbf{R}^{3}\right)$ (which is uniquely defined, up to a constant), such that $\widetilde{h}=\nabla \widetilde{g}$, still in the sense of distributions. In addition, since $\widetilde{g}_{\Lambda}$ satisfies

$$
-\Delta \widetilde{g}_{\Lambda}=-\operatorname{div}\left(\nabla \widetilde{g}_{\Lambda}\right)=4 \pi\left[\widetilde{\chi}_{\Gamma(\Lambda)}-\widetilde{\rho}_{\Lambda}\right],
$$

we deduce that $\widetilde{g}$ is a solution to

$$
-\operatorname{div}(\nabla \widetilde{g})=-\Delta \widetilde{g}=4 \pi[1-\widetilde{\rho}],
$$

in $\mathcal{D}^{\prime}\left(\mathbf{R}^{3}\right)$ (see [11]). With (4.31), we obtain

$$
\liminf _{\Lambda \rightarrow \infty} \frac{1}{|\Lambda|} D\left(\chi_{\Gamma(\Lambda)}-\rho_{\Lambda}, \chi_{\Gamma(\Lambda)}-\rho_{\Lambda}\right) \geqslant \int_{Q}|\nabla \widetilde{g}|^{2} .
$$

Now, we notice that we already know another solution to (4.32), namely

$$
\bar{g}(x)=\int_{Q} G(x-y)(1-\widetilde{\rho}(y)) d y=-\int_{Q} G(x-y) \widetilde{\rho}(y) d y,
$$

thanks to the normalization (2.14) on $G$. The function $\bar{g}$ is periodic and satisfies

$$
\int_{Q}|\nabla \bar{g}|^{2}=\int_{Q}-\Delta \bar{g} \cdot \bar{g}=D_{G}(\widetilde{\rho}, \widetilde{\rho}),
$$

thanks to (4.32), for $\int_{Q} \bar{g}=0$. We are going to show that

$$
\int_{Q}|\nabla \widetilde{g}|^{2} \geqslant \int_{Q}|\nabla \bar{g}|^{2}
$$

For this purpose, we first remark that $\partial_{1} \widetilde{g}-\partial_{1} \bar{g}$ (where $\partial_{1}$ denotes the first derivative with respect to the first coordinate $x_{1}$ of $\mathbf{R}^{3}$ ) is an harmonic function, for $\widetilde{g}$ and $\bar{g}$ are two solutions to the same Laplace equation (4.32).

Now, both $\partial_{1} \widetilde{g}$ (as the limit of $\partial_{1} \widetilde{g}_{\Lambda}$ ) and $\partial_{1} \bar{g}$ (by construction, see (4.33)) are in $L_{\text {unif }}^{2}\left(\mathbf{R}^{3}\right)$. Therefore, $\partial_{1} \widetilde{g}-\partial_{1} \bar{g}$ is an harmonic function which belongs to $L_{\text {unif }}^{2}\left(\mathbf{R}^{3}\right)$, and thus is a constant, that we denote by $a_{1}$ (use for instance the mean-value inequality). The same argument applies to the first derivatives with respect to the coordinates $x_{2}$ and $x_{3}$. Hence we obtain

$$
\widetilde{g}-\bar{g}=a \cdot x+b,
$$

where $a=\left(a_{1}, a_{2}, a_{3}\right)$ and $b$ are two fixed vectors of $\mathbf{R}^{3}$. It follows from this equality, that 


$$
\begin{aligned}
\int_{Q}|\nabla \widetilde{g}|^{2} & =\int_{Q}|\nabla(\bar{g}+a \cdot x+b)|^{2} \\
& =\int_{Q}|\nabla \bar{g}|^{2}+\int_{Q}|a|^{2}+2 \int_{Q} a \cdot \nabla \bar{g} \\
& =\int_{Q}|\nabla \bar{g}|^{2}+\int_{Q}|a|^{2} \\
& \geqslant \int_{Q}|\nabla \bar{g}|^{2},
\end{aligned}
$$

since

$$
\int_{Q} a \cdot \nabla \bar{g}=a \cdot \int_{Q} \nabla \bar{g}=0
$$

because of the periodicity of $\bar{g}$. The inequality (4.34) follows, from which we deduce easily (4.29) and (4.30).

At this stage, we collect (4.9) with (4.30) to obtain

$$
\begin{aligned}
\liminf _{\Lambda \rightarrow \infty} \frac{I_{\Lambda}^{R H F}}{|\Lambda|} & =\liminf _{\Lambda \rightarrow \infty} \frac{1}{|\Lambda|}\left(\operatorname{Tr}_{L^{2}\left(\mathbf{R}^{3}\right)}\left[\left(-\Delta-V_{\Lambda}\right) K_{\Lambda}\right]+\frac{1}{2} D\left(\rho_{\Lambda}, \rho_{\Lambda}\right)+\frac{1}{2} U_{\Lambda}\right) \\
& \geqslant \int_{Q^{\star}} \operatorname{Tr}_{L_{\xi}^{2}(Q)}\left[-\Delta_{\xi} \widetilde{K}_{\xi}\right] \frac{d \xi}{(2 \pi)^{3}}-\int_{Q} G \widetilde{\rho}+\frac{1}{2} D_{G}(\widetilde{\rho}, \widetilde{\rho})+\frac{M}{2} \\
& =E_{\mathrm{per}}^{R H F}(\widetilde{K})+\frac{M}{2} \\
& \geqslant I_{\mathrm{per}}^{R H F}+\frac{M}{2} .
\end{aligned}
$$

This concludes the proof of Proposition 4.1.

Before turning to the study of the upper limit of the energy per unit volume, we can anticipate a little bit and assume that we have already proven

$$
I_{\text {per }}^{R H F}+\frac{M}{2} \geqslant \limsup _{\Lambda \rightarrow \infty} \frac{1}{|\Lambda|} I_{\Lambda}^{R H F},
$$

which will be the purpose of Proposition 4.2 below. It will follow from the comparison of (4.35) and (4.36) that all equalities in these strings of inequalities (4.9), (4.30), (4.35), (4.36) are indeed equalities. In particular, we shall recover

$$
\lim _{\Lambda \rightarrow \infty} \frac{1}{|\Lambda|} \operatorname{Tr}_{L^{2}\left(\mathbf{R}^{3}\right)}\left[-\Delta K_{\Lambda}\right]=\int_{Q^{\star}} \operatorname{Tr}_{L_{\xi}^{2}(Q)}\left[-\Delta_{\xi} \widetilde{K}_{\xi}\right] \frac{d \xi}{(2 \pi)^{3}},
$$

and we shall also obtain that $\widetilde{K}$ is a minimizer of $I_{\text {per }}^{R H F}$.

In order to prove that the upper limit behaves in the expected way, we shall make use of the minimizer of (2.17) (it is not stricto sensu necessary, as we might use an almost minimizer). Therefore, we devote the next section to the study of problem (2.17), and 
in particular to the proof of Theorem 2.1. We shall come back to the proof of the upper limit (4.36), and thus conclude the proof of Theorem 2.2, in Subsection 4.3.

\subsection{The periodic RHF problem}

We begin this section with the proof of Theorem 2.1, that we recall here for the convenience of the reader.

THEOREM 2.1 (Well-posedness of the RHF periodic model). - The minimization problem defined by (2.17)-(2.18), i.e.

$$
\begin{gathered}
I_{\mathrm{per}}^{R H F}=\inf \left\{E_{\mathrm{per}}^{R H F}(K) ; K \in \mathcal{K}\right\}, \\
E_{\mathrm{per}}^{R H F}(K)=\int_{Q^{\star}} \operatorname{Tr}_{L_{\xi}^{2}(Q)}\left[-\Delta_{\xi} K_{\xi}\right] \frac{d \xi}{(2 \pi)^{3}}-\int_{Q} G \rho+\frac{1}{2} D_{G}(\rho, \rho)
\end{gathered}
$$

(respectively in the smeared nuclei case by Eqs. (2.19)-(2.20) in Section 2) admits a minimum. In addition, the minimizing density $\rho(x ; x)$ is unique and, thus, shares the symmetries of the unique cube.

Before we begin with the proof, let us at once remark that the argument we are going to make will be also useful in the Hartree-Fock case for the proof of Theorem 2.3 in Section 5 below. As the reduced Hartree-Fock model is convex, another strategy than the one we shall use below could have been chosen. However, we have chosen on purpose a strategy of proof that will be also valid for the non-convex Hartree-Fock model. This will simplify our task in Section 5. Let us also remark that we only do the proof in the point nuclei case, and that adapting our argument to the smeared nuclei case (2.19)(2.20) is straightforward. Let us also mention at this stage that some of our arguments are similar to those used by Lieb, Solovej and Yngvason in [34], where a close problem is studied.

Proof of Theorem 2.1. - Let us consider a minimizing sequence $K^{n}$ for the minimization problem (2.17)-(2.18). For each $n$, the operator $K^{n}$ may be decomposed into operators $K_{\xi}^{n}$. We denote by $\rho_{n}(\xi, x, y)$ the kernel of $K_{\xi}^{n}$, and by $\rho_{n}(x, y)=$ $\int_{Q^{\star}} \rho_{n}(\xi, x, y) \frac{d \xi}{(2 \pi)^{3}}$ the Schwartz kernel of $K_{n}$, according to Lemma 3.4. More precisely, we have, in view of Proposition 3.2,

$$
\rho_{n}(\xi, x, y)=\sum_{p \geqslant 1} \lambda_{p}^{(n)}(\xi) u_{p}^{(n)}(\xi, x) u_{p}^{(n)}(\xi, y)^{*} .
$$

In the right-hand side, the index $n$, referring to the index in the minimizing sequence $K^{n}$, has been put into parentheses in order to avoid ambiguity with powers of $u_{p}$. Since, in addition, $K_{\xi}^{n}$ is trace-class on $L_{\xi}^{2}(Q)$, we may also define

$$
\rho_{n}(\xi, x, x)=\sum_{p \geqslant 1} \lambda_{p}^{(n)}(\xi)\left|u_{p}^{(n)}(\xi, x)\right|^{2},
$$

which is a non-negative, periodic function in $L^{1}(Q)$, such that $\operatorname{Tr}_{L_{\xi}^{2}(Q)} K_{\xi}^{n}$ $=\int_{Q} \rho_{n}(\xi, x, x) d x$. Moreover, let us recall from Proposition 3.2, that the density $\rho(x ; x)$ 
which appears in the definition (2.18) of the energy functional is also a non-negative periodic function in $L^{1}(Q)$ (at least) defined by $\rho(x ; x)=\int_{Q^{\star}} \rho_{n}(\xi, x, x) \frac{d \xi}{(2 \pi)^{3}}$. Our first step consists in finding some bounds, independent of $n$, on the operators $K^{n}$ and on the functions $\rho_{n}(\cdot, \cdot)$ and $\rho_{n}(\xi, \cdot, \cdot)$.

Step 1. - A priori estimates on the minimizing sequence.

First of all, we remark that the following bound holds obviously

$$
0 \leqslant K_{\xi}^{n} \leqslant \mathbf{1}
$$

for almost every $\xi$ in $Q^{\star}$, which comes straightforwardly from Lemma 3.3. In view of the decomposition of the operators $K_{\xi}^{n}$ along their eigenbasis, let us rewrite the kinetic energy term:

$$
\int_{Q^{\star}} \operatorname{Tr}_{L_{\xi}^{2}(Q)}\left[-\Delta_{\xi} K_{\xi}^{n}\right] d \xi=\int_{Q^{\star}} \sum_{p \geqslant 1} \lambda_{p}^{(n)}(\xi) \int_{Q}\left|\nabla u_{p}^{(n)}(\xi, x)\right|^{2} d x d \xi .
$$

Next, we remark that the constraint of charge 1, namely

$$
\int_{Q^{\star}} \operatorname{Tr}_{L_{\xi}^{2}(Q)} K_{\xi}^{n} \frac{d \xi}{(2 \pi)^{3}}=1
$$

may also be written as

$$
\int_{Q^{\star}} \frac{d \xi}{(2 \pi)^{3}} \int_{Q} \rho_{n}(\xi ; x ; x) d x=\int_{Q^{\star}} \sum_{p \geqslant 1} \lambda_{p}^{(n)}(\xi) \frac{d \xi}{(2 \pi)^{3}}=\int_{Q} \rho_{n}(x ; x) d x=1 .
$$

Another consequence of this constraint is

$$
\begin{aligned}
& \int_{Q^{\star}} \frac{d \xi}{(2 \pi)^{3}} \iint_{Q \times Q}\left|\rho_{n}(\xi, x, y)\right|^{2} d x d y \\
& \quad=\int_{Q^{\star}} \sum_{p \geqslant 1} \sum_{q \geqslant 1} \lambda_{p}^{(n)}(\xi) \lambda_{q}^{(n)}(\xi)\left|\int_{Q} u_{p}^{(n)}(\xi, x) u_{q}^{(n)}(\xi, x)^{*} d x\right|^{2} \frac{d \xi}{(2 \pi)^{3}} \\
& =\int_{Q^{\star}} \sum_{p \geqslant 1} \sum_{q \geqslant 1} \lambda_{p}^{(n)}(\xi) \lambda_{q}^{(n)}(\xi) \delta_{p, q} \frac{d \xi}{(2 \pi)^{3}} \\
& =\int_{Q^{\star}} \sum_{p \geqslant 1}\left|\lambda_{p}^{(n)}(\xi)\right|^{2} \frac{d \xi}{(2 \pi)^{3}} \\
& \leqslant \int_{Q^{\star}} \sum_{p \geqslant 1} \lambda_{p}^{(n)}(\xi) \frac{d \xi}{(2 \pi)^{3}}=1,
\end{aligned}
$$

whence

$$
\rho_{n}(\xi, x, y) \text { is bounded in } L^{2}\left(Q^{\star} \times Q \times Q\right),
$$


or, equivalently, because of (3.26) in Lemma 3.4,

$$
\rho_{n}(x, y) \text { is bounded in } L^{2}\left(Q \times \mathbf{R}^{3}\right) .
$$

(Note that actually $\rho_{n}(x, y)$ is bounded in $L_{\text {unif }}^{2}\left(\mathbf{R}^{3} ; L^{2}\left(\mathbf{R}^{3}\right)\right.$ ), thanks to the translation invariance.) We are now going to work on the energy functional. Owing to the convexity of the function $f \mapsto \int_{Q}|\nabla \sqrt{f}|^{2}$, and because of (4.39), we have

$$
\begin{aligned}
\int_{Q}\left|\nabla \sqrt{\rho}_{n}(x, x)\right|^{2} d x & =\int_{Q}\left|\nabla\left(\int_{Q^{\star}} \sum_{p \geqslant 1} \lambda_{p}^{(n)}(\xi)\left|u_{p}^{(n)}(\xi, x)\right|^{2} \frac{d \xi}{(2 \pi)^{3}}\right)^{1 / 2}\right|^{2} d x \\
& \leqslant\left.\iint_{Q \times Q^{\star}} \sum_{p \geqslant 1} \lambda_{p}^{(n)}(\xi)|\nabla| u_{p}^{(n)}(\xi, x)\right|^{2} \frac{d x d \xi}{(2 \pi)^{3}} \\
& \leqslant \iint_{Q^{\star}} \sum_{p \geqslant 1} \lambda_{p}^{(n)}(\xi)\left|\nabla u_{p}^{(n)}(\xi, x)\right|^{2} \frac{d x d \xi}{(2 \pi)^{3}} \\
& =\int_{Q^{\star}} \operatorname{Tr}_{L_{\xi}^{2}(Q)}\left[-\Delta_{\xi} K_{\xi}^{n}\right] \frac{d \xi}{(2 \pi)^{3}},
\end{aligned}
$$

the second inequality being true since $|\nabla| f|| \leqslant|\nabla f|$ for any complex-valued function $f$. Let us now observe that

$$
\begin{aligned}
\left|\int_{Q} G(x) \rho_{n}(x, x) d x\right| & \leqslant C\|G\|_{L^{2}(Q)}\left\|\rho_{n}\right\|_{L^{1}(Q)}^{3 / 4}\left\|\rho_{n}\right\|_{L^{3}(Q)}^{1 / 4} \\
& \leqslant C\|G\|_{L^{2}(Q)}\left\|\sqrt{\rho}_{n}\right\|_{H^{1}(Q)}^{1 / 2} \\
& \leqslant C\|G\|_{L^{2}(Q)}\left(1+\left\|\nabla \sqrt{\rho}_{n}\right\|_{L^{2}(Q)}^{2}\right)^{1 / 4}
\end{aligned}
$$

since $G$ is $L^{2}$ on $Q$ (it has only a singularity like $\frac{1}{|x|}$ ) and $\left\|\rho_{n}\right\|_{L^{1}(Q)}=1$, and where $C$ denotes here and below some positive constant that is independent of $n$. Inserting (4.42) and (4.43) into the definition (2.18) of the energy, and noticing that $\frac{1}{2} D_{G}(\cdot, \cdot) \geqslant 0$, we obtain that we have, for the minimizing sequence $K^{n}$,

$$
\sqrt{\rho_{n}(x, x)} \text { is bounded in } H^{1}(Q) \text {, and thus in } L^{p}(Q), 1 \leqslant p \leqslant 6 \text {. }
$$

This can also be expressed by stating that

$$
\int_{Q^{\star}} \operatorname{Tr}_{L_{\xi}^{2}(Q)}\left[-\Delta_{\xi} K_{\xi}^{n}\right] d \xi=\iint_{Q \times Q^{\star}} \sum_{p \geqslant 1} \lambda_{p}^{(n)}(\xi)\left|\nabla u_{p}^{(n)}(\xi, y)\right|^{2} d x d \xi
$$

is bounded independently of $n$.

Using the Cauchy-Schwarz inequality as follows 


$$
\begin{aligned}
\left|\rho_{n}(x, y)\right|= & \left|\int_{Q^{\star}} \sum_{p \geqslant 1} \lambda_{p}^{(n)}(\xi) u_{p}^{(n)}(\xi, x) u_{p}^{(n)}(\xi, y)^{*} \frac{d \xi}{(2 \pi)^{3}}\right| \\
\leqslant & \left(\int_{Q^{\star}} \sum_{p \geqslant 1} \lambda_{p}^{(n)}(\xi)\left|u_{p}^{(n)}(\xi, x)\right|^{2} \frac{d \xi}{(2 \pi)^{3}}\right)^{1 / 2} \\
& \times\left(\int_{Q^{\star}} \sum_{p \geqslant 1} \lambda_{p}^{(n)}(\xi)\left|u_{p}^{(n)}(\xi, y)\right|^{2} \frac{d \xi}{(2 \pi)^{3}}\right)^{1 / 2} \\
= & \sqrt{\rho_{n}(x, x)} \sqrt{\rho_{n}(y, y)},
\end{aligned}
$$

we obtain a direct corollary of the bound (4.44):

$$
\rho_{n}(x, y) \text { is bounded in } L^{p}(Q \times Q), 1 \leqslant p \leqslant 6 \text {. }
$$

Another corollary of these bounds is obtained by using the convexity of the function $f \mapsto \iint_{Q \times Q^{\star}}|\nabla \sqrt{f}|^{2} d x d \xi$, it is

$$
\sqrt{\rho_{n}(\xi, x, x)} \text { is bounded in } L^{2}\left(Q^{\star} ; H^{1}(Q)\right) \text {. }
$$

Finally, a very useful bound is obtained from (4.45) by using the Lieb-Thirring inequality in this setting. (This is an easy adaptation of the Lieb-Thirring inequality in the periodic case given in the Appendix of [54] for finite-rank projectors together with the results of [39] for its extension to general density matrices.) We have, for almost every $\xi \in Q^{\star}$,

$$
\int_{Q} \rho_{n}(\xi, x, x)^{5 / 3} d x \leqslant C_{0} \operatorname{Tr}_{L_{\xi}^{2}(Q)}\left[\left(1-\Delta_{\xi}\right) K_{\xi}^{n}\right]
$$

for some constant $C_{0}$, which may be chosen independently of $\xi$, since $\xi$ lies in a bounded subset of $\mathbf{R}^{3}$, and therefore by integration on $Q^{\star}$ (since the left-hand side lies in $L^{1}\left(Q^{\star}\right)$ ),

$$
\int_{Q^{\star}} \frac{d \xi}{(2 \pi)^{3}} \int_{Q} \rho_{n}(\xi, x, x)^{5 / 3} d x \leqslant C \int_{Q^{\star}} \operatorname{Tr}_{L_{\xi}^{2}(Q)}\left[(1-\Delta)_{\xi} K_{\xi}^{n}\right] \frac{d \xi}{(2 \pi)^{3}} .
$$

This shows that

$$
\rho_{n}(\xi, x, x) \text { is bounded in } L^{5 / 3}\left(Q^{\star} \times Q\right),
$$

and concludes our first step, devoted to the a priori bounds on the sequence $K^{n}$.

Step 2. - Passing to the limit in the constraint.

Let us first remark that, in view of the bound (4.40), we may assume without loss of generality that the sequence $\rho_{n}(\xi, x, y)$ converges weakly in $L^{2}\left(Q^{\star} \times Q \times Q\right)$ to some $\rho_{\infty}(\xi, x, y)$. According to the formal decomposition given in [45], and recalled in Section 3.2, we may now define a self-adjoint operator $K^{\infty}$ on $L^{2}\left(\mathbf{R}^{3}\right)$, by $K^{\infty}=$ $\int_{Q^{\star}} K_{\xi}^{\infty} \frac{d \xi}{(2 \pi)^{3}}$, where $K_{\xi}^{\infty}$ is the Hilbert-Schmidt operator on $L_{\xi}^{2}(Q)$ whose kernel is 
the function $\rho_{\infty}(\xi, x, y)$. Another way to state the weak convergence of $\rho_{n}$ to $\rho_{\infty}$ is to say that for almost $\xi \in Q^{\star}$, and for any self-adjoint operator $L$ on $L^{2}\left(\mathbf{R}^{3}\right)$, such that $L=\int_{Q^{\star}} L_{\xi} \frac{d \xi}{(2 \pi)^{3}}$, where the operators $L_{\xi}$ are Hilbert-Schmidt operators on $L_{\xi}^{2}(Q)$, whose kernels $L(\xi, x, y)$ belong to $L^{2}\left(Q^{\star} ; L^{2}(Q \times Q)\right)$, we have

$$
\lim _{n \rightarrow+\infty} \int_{Q^{\star}} \operatorname{Tr}_{L_{\xi}^{2}(Q)}\left[K_{\xi}^{n} \cdot L_{\xi}^{*}\right] \frac{d \xi}{(2 \pi)^{3}}=\int_{Q^{\star}} \operatorname{Tr}_{L_{\xi}^{2}(Q)}\left[K_{\xi}^{\infty} \cdot L_{\xi}^{*}\right] \frac{d \xi}{(2 \pi)^{3}} .
$$

Clearly, the operators $K_{\xi}^{\infty}$ satisfy $0 \leqslant K_{\xi}^{\infty} \leqslant \mathbf{1}$, and thus $0 \leqslant K^{\infty} \leqslant \mathbf{1}$.

A second consequence of the bounds of Step 1 comes from (4.44). Again, we may always assume that the sequence $\sqrt{\rho_{n}(x, x)}$ converges weakly in $H_{\text {per }}^{1}(Q)$, strongly in $L^{p}(Q), 1 \leqslant p<6$, and almost everywhere on $\mathbf{R}^{3}$, to some function $\sqrt{\bar{\rho}_{\infty}(x, x)} \in$ $H_{\text {per }}^{1}(Q)$.

A third consequence of the bounds of Step 1 is deduced from (4.49): We may suppose that the sequence of (non-negative) functions $\rho_{n}(\xi, x, x)$ converges weakly in $L^{5 / 3}\left(Q^{\star} \times Q\right)$ to some (non-negative) function that we denote for the moment by $\bar{\rho}_{\infty}(\xi, x, x)$.

Let us first prove that $\int_{Q^{\star}} \bar{\rho}_{\infty}(\xi, x, x) \frac{d \xi}{(2 \pi)^{3}}=\bar{\rho}_{\infty}(x, x)$. For this purpose, we note that the weak convergence in $L^{5 / 3}\left(Q^{\star} \times Q\right)$ implies in particular that, for any function $v \in L^{5 / 2}(Q)$,

$$
\lim _{n \rightarrow+\infty} \int_{Q^{\star}} \frac{d \xi}{(2 \pi)^{3}} \int_{Q} \rho_{n}(\xi, x, x) v(x) d x=\int_{Q^{\star}} \frac{d \xi}{(2 \pi)^{3}} \int_{Q} \bar{\rho}_{\infty}(\xi, x, x) v(x) d x .
$$

Now, the left-hand side is also given by

$$
\lim _{n \rightarrow+\infty} \int_{Q}\left(\int_{Q^{\star}} \rho_{n}(\xi, x, x) \frac{d \xi}{(2 \pi)^{3}}\right) v(x) d x=\lim _{n \rightarrow+\infty} \int_{Q} \rho_{n}(x, x) v(x) d x,
$$

and thus by

$$
\lim _{n \rightarrow+\infty} \int_{Q} \rho_{n}(x, x) v(x) d x=\int_{Q} \bar{\rho}_{\infty}(x, x) v(x) d x .
$$

Therefore, we have

$$
\int_{Q^{\star}} \bar{\rho}_{\infty}(\xi, x, x) \frac{d \xi}{(2 \pi)^{3}}=\bar{\rho}_{\infty}(x, x) .
$$

At this stage, we do not know a priori that $\rho_{\infty}(\xi, x, x)=\bar{\rho}_{\infty}(\xi, x, x)$, but we shall prove this claim below in Step 3 .

Let us now turn to the proof of the fact that the operator $K^{\infty}$ necessarily satisfies the constraint:

$$
\int_{Q^{\star}} \operatorname{Tr}_{L_{\xi}^{2}(Q)} K_{\xi}^{\infty} \frac{d \xi}{(2 \pi)^{3}}=1
$$


The difficulty to deduce (4.52), from the convergence (4.50) and the fact that the above constraint is satisfied for all $n$, is of course that we cannot take $L$ as being the identity operator in (4.50), for the identity is not a Hilbert-Schmidt operator. In order to conclude, we shall need to use the bound on the kinetic energy term. We argue as follows.

For all $n$, and for almost all $\xi \in Q^{\star}$, we know that $K_{\xi}^{n}$ and $-\Delta_{\xi} K_{\xi}^{n}$ are trace-class operators on $L_{\xi}^{2}(Q)$. In particular, this implies that the operator $(1-\Delta)_{\xi}^{1 / 2} K_{\xi}^{n}(1-\Delta)_{\xi}^{1 / 2}$ is also trace-class (thus, in particular, Hilbert-Schmidt). In addition, since we have a bound, derived from (4.45) and (4.38),

$$
\int_{Q^{\star}} \operatorname{Tr}_{L_{\xi}^{2}(Q)}\left[(1-\Delta)_{\xi}^{1 / 2} K_{\xi}^{n}(1-\Delta)_{\xi}^{1 / 2}\right] d \xi=\int_{Q^{\star}} \operatorname{Tr}_{L_{\xi}^{2}(Q)}\left[(1-\Delta)_{\xi} K_{\xi}^{n}\right] d \xi \leqslant C,
$$

we may assume, extracting a subsequence if necessary, that the sequence of operators $\int_{Q^{\star}}(1-\Delta)_{\xi}^{1 / 2} K_{\xi}^{n}(1-\Delta)_{\xi}^{1 / 2} \frac{d \xi}{(2 \pi)^{3}}$ converges in the sense of (4.50), and its limit is necessarily $\int_{Q^{\star}}(1-\Delta)_{\xi}^{1 / 2} K_{\xi}^{\infty}(1-\Delta)_{\xi}^{1 / 2} \frac{d \xi}{(2 \pi)^{3}}$. Testing this weak convergence with the operators $L_{\xi}=(1-\Delta)_{\xi}^{-1}$, which are Hilbert-Schmidt on $L_{\xi}^{2}(Q)$, we obtain

$$
\begin{array}{r}
\lim _{n \rightarrow+\infty} \int_{Q^{\star}} \operatorname{Tr}_{L_{\xi}^{2}(Q)}\left[(1-\Delta)_{\xi}^{1 / 2} K_{\xi}^{n}(1-\Delta)_{\xi}^{1 / 2}(1-\Delta)_{\xi}^{-1}\right] d \xi \\
=\int_{Q^{\star}} \operatorname{Tr}_{L_{\xi}^{2}(Q)}\left[(1-\Delta)_{\xi}^{1 / 2} K_{\xi}^{\infty}(1-\Delta)_{\xi}^{1 / 2}(1-\Delta)_{\xi}^{-1}\right] d \xi ;
\end{array}
$$

that is

$$
\lim _{n \rightarrow+\infty} \int_{Q^{\star}} \operatorname{Tr}_{L_{\xi}^{2}(Q)} K_{\xi}^{n} d \xi=\int_{Q^{\star}} \operatorname{Tr}_{L_{\xi}^{2}(Q)} K_{\xi}^{\infty} d \xi .
$$

Therefore, as $\int_{Q^{\star}} \operatorname{Tr}_{L_{\xi}^{2}(Q)} K_{\xi}^{n} \frac{d \xi}{(2 \pi)^{3}}=1$ for all $n$, we deduce that the operator $K^{\infty}$ satisfies the constraint.

Step 3. - Passing to the limit in the energy.

A simple argument, using the operator $(1-\Delta)$ and Fatou's lemma allows one to show, arguing as in the proof of Proposition 4.1, and making use of Step 2, that

$$
\begin{aligned}
& \liminf _{n \rightarrow+\infty}\left[1+\int_{Q^{\star}} \operatorname{Tr}_{L_{\xi}^{2}(Q)}\left[-\Delta_{\xi} K_{\xi}^{n}\right] d \xi\right] \\
& =\liminf _{n \rightarrow+\infty} \int_{Q^{\star}} \operatorname{Tr}_{L_{\xi}^{2}(Q)}\left[\left(1-\Delta_{\xi}\right) K_{\xi}^{n}\right] d \xi \\
& \geqslant \lim _{n \rightarrow+\infty} \int_{Q^{\star}} \operatorname{Tr}_{L_{\xi}^{2}(Q)}\left[\left(1-\Delta_{\xi}\right) K_{\xi}^{\infty}\right] \frac{d \xi}{(2 \pi)^{3}} \\
& =1+\int_{Q^{\star}} \operatorname{Tr}_{L_{\xi}^{2}(Q)}\left[-\Delta_{\xi} K_{\xi}^{\infty}\right] \frac{d \xi}{(2 \pi)^{3}},
\end{aligned}
$$

and, therefore, that 


$$
\liminf _{n \rightarrow+\infty} \int_{Q^{\star}} \operatorname{Tr}_{L_{\xi}^{2}(Q)}\left[-\Delta_{\xi} K_{\xi}^{n}\right] \frac{d \xi}{(2 \pi)^{3}} \geqslant \int_{Q^{\star}} \operatorname{Tr}_{L_{\xi}^{2}(Q)}\left[-\Delta_{\xi} K_{\xi}^{\infty}\right] \frac{d \xi}{(2 \pi)^{3}} .
$$

Next, a standard argument on the sequence $\rho_{n}(x, x)$, whose square root converges in $H_{\text {per }}^{1}(Q)$, shows that

$$
\begin{gathered}
\lim _{n \rightarrow+\infty}\left[-\int_{Q} G(x) \rho_{n}(x, x) d x+\frac{1}{2} D_{G}\left(\rho_{n}(x, x), \rho_{n}(x, x)\right)\right] \\
=-\int_{Q} G(x) \bar{\rho}_{\infty}(x, x) d x+\frac{1}{2} D_{G}\left(\bar{\rho}_{\infty}(x, x), \bar{\rho}_{\infty}(x, x)\right) .
\end{gathered}
$$

It is then clear that (4.53) along with (4.54) will suffice to establish the existence of a minimizer for the periodic RHF model, provided we are able to show that, for almost every $\xi \in Q^{\star}$ and $x \in Q$,

$$
\bar{\rho}_{\infty}(\xi, x, x)=\rho_{\infty}(\xi, x, x) .
$$

We finally prove this fact. For this purpose, we choose an arbitrary function $\theta(\xi, x) \in$ $L^{\infty}\left(Q^{\star} \times Q\right)$, and define the Hilbert-Schmidt operators

$$
L_{\xi}=(1-\Delta)_{\xi}^{-1 / 2} \theta(\xi, \cdot)(1-\Delta)_{\xi}^{-1 / 2} .
$$

Using the convergence (4.50) for the sequence of operators $(1-\Delta)_{\xi}^{1 / 2} K_{\xi}^{n}(1-\Delta)_{\xi}^{1 / 2}$, we have

$$
\begin{array}{r}
\lim _{n \rightarrow+\infty} \int_{Q^{\star}} \operatorname{Tr}_{L_{\xi}^{2}(Q)}\left[(1-\Delta)_{\xi}^{1 / 2} K_{\xi}^{n}(1-\Delta)_{\xi}^{1 / 2}(1-\Delta)_{\xi}^{-1 / 2} \theta(\xi, \cdot)(1-\Delta)_{\xi}^{-1 / 2}\right] d \xi \\
=\int_{Q^{\star}} \operatorname{Tr}_{L_{\xi}^{2}(Q)}\left[(1-\Delta)_{\xi}^{1 / 2} K_{\xi}^{\infty}(1-\Delta)_{\xi}^{1 / 2}(1-\Delta)_{\xi}^{-1 / 2} \theta(\xi, \cdot)(1-\Delta)_{\xi}^{-1 / 2}\right] d \xi .
\end{array}
$$

This may also read

$$
\lim _{n \rightarrow+\infty} \iint_{Q^{\star} \times Q} \rho_{n}(\xi, x, x) \theta(\xi, x) d x d \xi=\iint_{Q^{\star} \times Q} \rho_{\infty}(\xi, x, x) \theta(\xi, x) d x d \xi .
$$

But, on the other hand, we know that the sequence of functions $\rho_{n}(\xi, x, x)$ converges weakly in $L^{5 / 3}\left(Q^{\star} \times Q\right)$ to $\bar{\rho}_{\infty}(\xi, x, x)$, thus we also have

$$
\lim _{n \rightarrow+\infty} \iint_{Q^{\star} \times Q} \rho_{n}(\xi, x, x) \theta(\xi, x) d x d \xi=\iint_{Q^{\star} \times Q} \bar{\rho}_{\infty}(\xi, x, x) \theta(\xi, x) d x d \xi .
$$

This shows equality (4.55), and concludes the proof of the existence of a minimum. We now show the uniqueness of the periodic density $\rho(x ; x)$. The argument is an adaptation of a similar claim in [34]. Assume that there exist two minimizers $K_{1}$ and $K_{2}$ in $\mathcal{K}$ of $I_{\text {per }}^{R H F}$. Denoting by $\rho_{1}$ and $\rho_{2}$ their respective density, it is easily checked that

$$
\begin{aligned}
E_{\mathrm{per}}^{R H F}(K) & =\frac{1}{2} E_{\mathrm{per}}^{R H F}\left(K_{1}\right)+\frac{1}{2} E_{\mathrm{per}}^{R H F}\left(K_{2}\right)-\frac{1}{8} D_{G}\left(\rho_{1}-\rho_{2}, \rho_{1}-\rho_{2}\right) \\
& =I_{\mathrm{per}}^{R H F}-\frac{1}{8} D_{G}\left(\rho_{1}-\rho_{2}, \rho_{1}-\rho_{2}\right) .
\end{aligned}
$$


We thus have $D_{G}\left(\rho_{1}-\rho_{2}, \rho_{1}-\rho_{2}\right)=0$. Now, since $G, \rho_{1}$ and $\rho_{2}$ are periodic, we may rewrite $D_{G}\left(\rho_{1}-\rho_{2}, \rho_{1}-\rho_{2}\right)$ with the help of the Fourier coefficients of $\rho_{1}-\rho_{2}$ and $G$. Since the Fourier series expansion of $G$ writes (see [32])

$$
G(x)=\frac{1}{\pi} \sum_{n \in \mathbf{Z}^{3} \backslash\{0\}} \frac{1}{|n|^{2}} \mathrm{e}^{2 i \pi n \cdot x},
$$

we observe that $D_{G}\left(\rho_{1}-\rho_{2}, \rho_{1}-\rho_{2}\right)=0$ if and only if $\rho_{1}-\rho_{2}$ is constant. But, $\int_{Q} \rho_{1}-\rho_{2}=0$, hence $\rho_{1}=\rho_{2}$. In particular, in the above proof, the whole sequence $\sqrt{\rho_{n}(x ; x)}$ converges to $\sqrt{\rho_{\infty}(x ; x)}$ and not only a subsequence.

Before concluding this section, let us write down the Euler-Lagrange equations satisfied by a minimizer $K$ of $I_{\text {per }}^{R H F}$. Using the decomposition of $K$ in $\mathcal{K}$ along an eigenbasis of each $K_{\xi}$, when $\xi$ describes $Q^{\star}$, we may reformulate the minimization problem $I_{\text {per }}^{R H F}$ in the following way: $I_{\text {per }}^{R H F}$ is obtained by minimizing

$$
\begin{aligned}
& \int_{Q^{\star}} \sum_{n \geqslant 1} \lambda_{n}(\xi) \int_{Q}\left(\left|\nabla u_{n}(\xi, x)\right|^{2}-G(x)\left|u_{n}(\xi, x)\right|^{2}\right) \frac{d x d \xi}{(2 \pi)^{3}} \\
& \quad+\frac{1}{2} \iint_{Q^{\star} \times Q^{\star}} \frac{d \xi d \xi^{\prime}}{(2 \pi)^{6}} \sum_{n, m \geqslant 1} \lambda_{n}(\xi) \lambda_{m}\left(\xi^{\prime}\right) \iint_{Q \times Q}\left|u_{n}(\xi, x)\right|^{2} G(x-y)\left|u_{m}\left(\xi^{\prime}, y\right)\right|^{2} d x d y
\end{aligned}
$$

subject to the constraints

$$
\left\{\begin{array}{l}
\int_{Q^{\star}} \sum_{n \geqslant 1} \lambda_{n}(\xi) \frac{d \xi}{(2 \pi)^{3}}=1, \\
0 \leqslant \lambda_{n}(\xi) \leqslant 1, \text { for all } n \geqslant 1, \text { and for almost all } \xi \in Q^{\star}, \\
\int_{Q} u_{n}(\xi, x) u_{m}^{*}(\xi, x) d x=\delta_{n, m}, \text { for almost all } \xi \in Q^{\star} .
\end{array}\right.
$$

The Euler-Lagrange equations satisfied by a minimizer $K$ of $I_{\text {per }}^{R H F}$ can then be easily written. They exhibit the Lagrange multipliers $\pi, \mu_{n}^{0}(\xi), \mu_{n}^{1}(\xi), \varepsilon_{n m}(\xi)$, respectively associated to the constraints of (4.56). More precisely, we obtain, for almost every $\xi$ in $Q^{\star}$, and for every $n \geqslant 1$,

$$
\left\{\begin{array}{l}
-\Delta u_{n}(\xi, \cdot)-G u_{n}(\xi, \cdot)+\sum_{m \geqslant 1} \lambda_{m}(\xi)\left(u_{m}(\xi, \cdot)^{2} \chi_{Q} \star G\right) u_{n}(\xi, \cdot) \\
=\sum_{m \geqslant 1} \varepsilon_{n m}(\xi) u_{m}(\xi, \cdot), \quad \text { a.e. on } Q, \\
\int_{Q}\left(\left|\nabla u_{n}(\xi, x)\right|^{2}-G(x)\left|u_{n}(\xi, x)\right|^{2}\right) d x \\
\quad+\sum_{m \geqslant 1} \lambda_{m}(\xi)\left(u_{m}(\xi, \cdot)^{2} \chi_{Q} \star G\right) u_{n}(\xi, \cdot) \\
=\mu_{n}^{0}(\xi)+\mu_{n}^{1}(\xi)+\pi .
\end{array}\right.
$$


Since $E_{\mathrm{per}}^{R H F}(K)$ is independent of the choice of an eigenbasis for $K_{\xi}$, we may assume without loss of generality that the hermitian matrix with entries $\varepsilon_{n m}(\xi)$ is diagonal, for almost every $\xi$ in $Q^{\star}$; in other words, the right-hand side term $\sum_{m \geqslant 1} \varepsilon_{n m}(\xi) u_{m}(\xi, \cdot)$ in the first equation of (4.57) may be replaced by $\varepsilon_{n}(\xi) u_{n}(\xi, \cdot)$. Moreover, owing to the fact that the Lagrange multipliers $\mu_{n}^{0}(\xi)$ and $\mu_{n}^{1}(\xi)$ are respectively associated to the constraints $0 \leqslant \lambda_{n}(\xi)$ and $\lambda_{n}(\xi) \leqslant 1$, they satisfy, for all $n \geqslant 1$ and for almost every $\xi$ in $Q^{\star}$,

$$
\begin{aligned}
& \mu_{n}^{0}(\xi) \begin{cases}=0, & \text { if } \lambda_{n}(\xi)>0, \\
\geqslant 0, & \text { if } \lambda_{n}(\xi)=0,\end{cases} \\
& \mu_{n}^{1}(\xi) \begin{cases}=0, & \text { if } \lambda_{n}(\xi)<1, \\
\leqslant 0, & \text { if } \lambda_{n}(\xi)=1 .\end{cases}
\end{aligned}
$$

We now apply $u_{n}(\xi, \cdot)$ to the first equation of (4.57), next integrate over $Q$, and, finally, insert the result into the second equation of (4.57), to obtain, using (4.58a) and (4.58b),

$$
\left\{\begin{array}{l}
\lambda_{n}(\xi)=0 \quad \Longrightarrow \varepsilon_{n}(\xi) \geqslant \pi, \\
0<\lambda_{n}(\xi)<1 \\
\lambda_{n}(\xi)=1 \quad \Longrightarrow \varepsilon_{n}(\xi)=\pi,
\end{array}\right.
$$

\subsection{Upper limit of the energy per unit volume and conclusion}

In order to conclude the proof of Theorem 2.2, we now prove the

Proposition 4.2. - We assume that the Van Hove sequence $\Lambda$ satisfies (2.21), and that the unit cell $Q$ is a cube. Then,

$$
\limsup _{\Lambda \rightarrow \infty} \frac{I_{\Lambda}^{R H F}}{|\Lambda|} \leqslant I_{\mathrm{per}}^{R H F}+\frac{M}{2},
$$

where $I_{\mathrm{per}}^{R H F}$ is defined by (2.17)-(2.18).

Remark 4.1. - As stated in Theorem 2.2, the same result holds true in the smeared nuclei case, if we assume moreover that $m$ shares the symmetries of the unit cube $Q$, and define $M$ in a convenient way.

As a corollary of Proposition 4.1 and Proposition 4.2 (and the slight modifications which are necessary to treat the smeared nuclei case), we shall obtain Theorem 2.2.

Proof of Proposition 4.2. - Let us denote by $K$ a minimizer of the periodic RHF problem. As usual, we may decompose $K$ into operators $K_{\xi}\left(\xi \in Q^{\star}\right)$, whose kernels $\rho(\xi, x, y)$ may be written as

$$
\rho(\xi, x, y)=\sum_{n \geqslant 1} \lambda_{n}(\xi) u_{n}(\xi, x) u_{n}(\xi, y)^{*}
$$


We denote

$$
\rho(x, y)=\int_{Q^{\star}} \rho(\xi, x, y) \frac{d \xi}{(2 \pi)^{3}} .
$$

Let now $\Lambda$ be fixed. We build a cut-off function $\chi_{\Lambda} \in \mathcal{D}\left(\mathbf{R}^{3}\right)$ satisfying the following properties:

$$
\left\{\begin{array}{l}
0 \leqslant \chi_{\Lambda} \leqslant 1 ; \\
\chi_{\Lambda} \equiv 1, \quad \text { on }\{x \in \Gamma(\Lambda) ; d(x, \partial \Gamma(\Lambda)) \geqslant 2\} \\
\chi_{\Lambda} \equiv 0, \quad \text { on } \Gamma(\Lambda)^{c} .
\end{array}\right.
$$

In addition, we choose $\chi_{\Lambda}$ in such a way that it also satisfies

$$
\int_{\mathbf{R}^{3}} \chi_{\Lambda}^{2}(x) \rho(x ; x) d x=|\Lambda|+\mathrm{o}(|\Lambda|) .
$$

We next consider the operator $K_{\Lambda}$ on $L^{2}\left(\mathbf{R}^{3}\right)$ whose kernel is

$$
\rho_{\Lambda}(x, y)=\chi_{\Lambda}(x) \rho(x, y) \chi_{\Lambda}(y)
$$

A simple computation shows that

$$
\left(K_{\Lambda} \psi ; \psi\right)_{L^{2}\left(\mathbf{R}^{3}\right)}=\left(K\left(\chi_{\Lambda} \psi\right) ;\left(\chi_{\Lambda} \psi\right)\right)_{L^{2}\left(\mathbf{R}^{3}\right)},
$$

and therefore we have $0 \leqslant K_{\Lambda} \leqslant \mathbf{1}$. The choice of $\chi_{\Lambda}$ ensures also that $\operatorname{Tr}_{L^{2}\left(\mathbf{R}^{3}\right)} K_{\Lambda}=$ $|\Lambda|+o(|\Lambda|) \leqslant|\Lambda|$.

We now compute the RHF energy of $K_{\Lambda}$.

Since $\rho_{\Lambda}(x ; x)=\chi_{\Lambda}^{2}(x) \rho(x ; x)$, it is a simple matter, arguing as in [11] and using the periodicity of $\rho(x ; x)$, to show that the electrostatic terms

$$
-\int_{\mathbf{R}^{3}} V_{\Lambda} \rho_{\Lambda}+\frac{1}{2} D\left(\rho_{\Lambda}, \rho_{\Lambda}\right)+\frac{1}{2} U_{\Lambda}
$$

behave like

$$
|\Lambda|\left[-\int_{Q} G \rho+\frac{1}{2} D_{G}(\rho, \rho)+\frac{M}{2}\right],
$$

as $\Lambda$ goes to infinity. This is precisely where we need the assumption (2.21), the fact that $Q$ is a cube, and that $\rho(x ; x)$ shares the symmetries of the unit cube. Both facts play a fundamental role - see the details in [11]. Therefore, we concentrate ourselves on the behaviour of the kinetic energy term. We intend to prove that

$$
\lim _{\Lambda \rightarrow \infty} \frac{1}{|\Lambda|} \operatorname{Tr}_{L^{2}\left(\mathbf{R}^{3}\right)}\left[-\Delta K_{\Lambda}\right]=\int_{Q^{\star}} \operatorname{Tr}_{L_{\xi}^{2}(Q)}\left[-\Delta_{\xi} K_{\xi}\right] \frac{d \xi}{(2 \pi)^{3}}
$$

which will of course conclude the proof of Proposition 4.2. 
Let us denote by $\left(\varphi_{m}\right)_{m \geqslant 1}$ an Hilbertian basis of $L^{2}\left(\mathbf{R}^{3}\right)$. We begin with

$$
\begin{aligned}
-\Delta K_{\Lambda} \varphi_{m} & =-\Delta\left(\int_{\mathbf{R}^{3}} \rho_{\Lambda}(x, y) \varphi_{m}(y) d y\right) \\
& =\int_{\mathbf{R}^{3}}-\Delta\left(\rho(x, y) \chi_{\Lambda}(x)\right) \chi_{\Lambda}(y) \varphi_{m}(y) d y \\
& =-\iint_{\mathbf{R}^{3} \times Q^{\star}} \sum_{n \geqslant 1} \lambda_{n}(\xi) \Delta\left(u_{n}(\xi, x) \chi_{\Lambda}(x)\right) u_{n}(\xi, y)^{*} \chi_{\Lambda}(y) \varphi_{m}(y) \frac{d y d \xi}{(2 \pi)^{3}} .
\end{aligned}
$$

Hence,

$$
\begin{aligned}
& \left(-\Delta K_{\Lambda} \varphi_{m} ; \varphi_{m}\right)_{L^{2}\left(\mathbf{R}^{3}\right)} \\
& \quad=\iint_{\mathbf{R}^{3} \times Q^{\star}} \sum_{n \geqslant 1} \lambda_{n}(\xi)\left[\int_{\mathbf{R}^{3}}-\Delta\left(u_{n}(\xi, x) \chi_{\Lambda}(x)\right) \varphi_{m}^{*}(x) d x\right] u_{n}(\xi, y)^{*} \chi_{\Lambda}(y) \varphi_{m}(y) \frac{d y d \xi}{(2 \pi)^{3}} .
\end{aligned}
$$

Summing up next in $m$, we obtain

$$
\begin{aligned}
\sum_{m \geqslant 1} & \left(-\Delta K_{\Lambda} \varphi_{m} ; \varphi_{m}\right)_{L^{2}\left(\mathbf{R}^{3}\right)} \\
= & \int_{\mathbf{R}^{3}} d y \int_{Q^{\star}} \sum_{n \geqslant 1} \lambda_{n}(\xi) u_{n}(\xi, y)^{*} \chi_{\Lambda}(y) \\
& \times \sum_{m \geqslant 1}\left[\int_{\mathbf{R}^{3}}-\Delta\left(u_{n}(\xi, x) \chi_{\Lambda}(x)\right) \varphi_{m}^{*}(x) d x\right] \varphi_{m}(y) \frac{d \xi}{(2 \pi)^{3}} \\
= & \int_{\mathbf{R}^{3}} d y \int_{Q^{\star}} \sum_{n \geqslant 1} \lambda_{n}(\xi) u_{n}(\xi, y)^{*} \chi_{\Lambda}(y)-\Delta\left(u_{n}(\xi, y) \chi_{\Lambda}(y)\right) \frac{d \xi}{(2 \pi)^{3}} \\
= & \int_{Q^{\star}} \frac{d \xi}{(2 \pi)^{3}} \sum_{n \geqslant 1} \lambda_{n}(\xi) \int_{\mathbf{R}^{3}} u_{n}(\xi, y)^{*} \chi_{\Lambda}(y)-\Delta\left(u_{n}(\xi, y) \chi_{\Lambda}(y)\right) d y \\
= & \int_{Q^{\star}} \frac{d \xi}{(2 \pi)^{3}} \sum_{n \geqslant 1} \lambda_{n}(\xi) \int_{\mathbf{R}^{3}}\left|\nabla\left(u_{n}(\xi, y) \chi_{\Lambda}(y)\right)\right|^{2} d y .
\end{aligned}
$$

We have therefore obtained

$$
\operatorname{Tr}_{L^{2}\left(\mathbf{R}^{3}\right)}\left[-\Delta K_{\Lambda}\right]=\int_{Q^{\star}} \frac{d \xi}{(2 \pi)^{3}} \sum_{n \geqslant 1} \lambda_{n}(\xi) \int_{\mathbf{R}^{3}}\left|\nabla\left(u_{n}(\xi, x) \chi_{\Lambda}(x)\right)\right|^{2} d x
$$

We now remark that

$$
\begin{aligned}
& \int_{\mathbf{R}^{3}}\left.\nabla\left(u_{n}(\xi, x) \chi_{\Lambda}(x)\right)\right|^{2} d x \\
& \quad=\int_{\mathbf{R}^{3}}\left|\chi_{\Lambda}(x) \nabla\left(u_{n}(\xi, x)\right)+u_{n}(\xi, x) \nabla \chi_{\Lambda}(x)\right|^{2} d x
\end{aligned}
$$




$$
\begin{aligned}
& =\int_{\mathbf{R}^{3}}\left|\chi_{\Lambda}(x)\right|^{2}\left|\nabla\left(u_{n}(\xi, x)\right)\right|^{2}+\int_{\mathbf{R}^{3}}\left|u_{n}(\xi, x)\right|^{2}\left|\nabla \chi_{\Lambda}(x)\right|^{2} d x \\
& \quad+2 \int_{\mathbf{R}^{3}} \chi_{\Lambda}(x) u_{n}^{*}(\xi, x) \nabla u_{n}(\xi, x) \cdot \nabla \chi_{\Lambda}(x) d x .
\end{aligned}
$$

Each of the three integrals in the right-hand side may be restricted on $\Gamma(\Lambda)$, for $\chi_{\Lambda}$ vanishes outside this domain. In addition, the third integral may be restricted on the "boundary" $\partial \Lambda=\{x \in \Gamma(\Lambda) ; d(x ; \partial \Gamma(\Lambda)) \leqslant 2\}$ of $\Gamma(\Lambda)$, as $\nabla \chi_{\Lambda} \equiv 0$ in the "interior" $\Gamma(\Lambda) \backslash \partial \Lambda$ of $\Gamma(\Lambda)$. Therefore, we have

$$
\int_{Q^{\star}} \frac{d \xi}{(2 \pi)^{3}} \sum_{n \geqslant 1} \lambda_{n}(\xi) \int_{\mathbf{R}^{3}}\left|\nabla\left(u_{n}(\xi, x) \chi_{\Lambda}(x)\right)\right|^{2} d x=I_{\Lambda}^{1}+I_{\Lambda}^{2}+2 I_{\Lambda}^{3},
$$

where $I_{\Lambda}^{1}, I_{\Lambda}^{2}, I_{\Lambda}^{3}$ denote respectively

$$
\begin{aligned}
& I_{\Lambda}^{1}=\int_{Q^{\star}} \frac{d \xi}{(2 \pi)^{3}} \sum_{n \geqslant 1} \lambda_{n}(\xi) \sum_{k \in \Lambda} \int_{Q}\left|\chi_{\Lambda}(x+k)\right|^{2}\left|\nabla\left(u_{n}(\xi, x+k)\right)\right|^{2} d x, \\
& I_{\Lambda}^{2}=\int_{Q^{\star}} \frac{d \xi}{(2 \pi)^{3}} \sum_{n \geqslant 1} \lambda_{n}(\xi) \sum_{k \in \Lambda} \int_{Q}\left|u_{n}(\xi, x+k)\right|^{2}\left|\nabla \chi_{\Lambda}(x+k)\right|^{2} d x, \\
& I_{\Lambda}^{3}=\int_{Q^{\star}} \frac{d \xi}{(2 \pi)^{3}} \sum_{n \geqslant 1} \lambda_{n}(\xi) \int_{\partial \Lambda} \chi_{\Lambda}(x) u_{n}^{*}(\xi, x) \nabla u_{n}(\xi, x) \cdot \nabla \chi_{\Lambda}(x) d x .
\end{aligned}
$$

As $\Lambda$ is a Van Hove sequence, we expect that the integrals over the "boundary" of $\Gamma(\Lambda)$ are negligible with respect to $|\Lambda|$, or, in other words, that only the integrals over the "interior" of $\Gamma(\Lambda)$ play a role in identifying the limit per unit volume. Indeed, it is easy to see, by a standard argument that we have already used in [11] and that is based upon the properties of $\chi_{\Lambda}$ and of $u_{n}(\xi, \cdot)$, that

$$
\begin{aligned}
I_{\Lambda}^{1} & =|\Lambda| \int_{Q^{\star}} \frac{d \xi}{(2 \pi)^{3}} \sum_{n \geqslant 1} \lambda_{n}(\xi) \int_{Q}\left|\nabla\left(u_{n}(\xi, x)\right)\right|^{2} d x+\mathrm{o}(|\Lambda|) \\
& =|\Lambda| \int_{Q^{\star}} \operatorname{Tr}_{L_{\xi}^{2}(Q)}\left[-\Delta_{\xi} K_{\xi}\right] \frac{d \xi}{(2 \pi)^{3}}+\mathrm{o}(|\Lambda|),
\end{aligned}
$$

and

$$
I_{\Lambda}^{2}=\mathrm{o}(|\Lambda|) \int_{Q^{\star}} \operatorname{Tr}_{L_{\xi}^{2}(Q)}\left[-\Delta_{\xi} K_{\xi}\right] \frac{d \xi}{(2 \pi)^{3}} .
$$

Finally, we may bound $I_{\Lambda}^{3}$ as follows

$$
\left|I_{\Lambda}^{3}\right| \leqslant \mathrm{o}(|\Lambda|) \int_{Q^{\star}} \sum_{n \geqslant 1} \lambda_{n}(\xi)\left(\int_{Q}\left|\nabla\left(u_{n}(\xi, x)\right)\right|^{2}\right)^{1 / 2}\left(\int_{Q}\left|u_{n}(\xi, x)\right|^{2}\right)^{1 / 2} d \xi
$$




$$
\begin{aligned}
\leqslant & \mathrm{o}(|\Lambda|) \int_{Q^{\star}}\left(\sum_{n \geqslant 1} \lambda_{n}(\xi) \int_{Q}\left|\nabla\left(u_{n}(\xi, x)\right)\right|^{2} d x\right)^{1 / 2}\left(\sum_{n \geqslant 1} \lambda_{n}(\xi) \int_{Q}\left|u_{n}(\xi, x)\right|^{2} d x\right)^{1 / 2} d \xi \\
\leqslant & o(|\Lambda|)\left(\int_{Q^{\star}} \sum_{n \geqslant 1} \lambda_{n}(\xi) \int_{Q}\left|\nabla\left(u_{n}(\xi, x)\right)\right|^{2} d x d \xi\right)^{1 / 2} \\
& \times\left(\int_{Q^{\star}} \sum_{n \geqslant 1} \lambda_{n}(\xi) \int_{Q}\left|u_{n}(\xi, x)\right|^{2} d x d \xi\right)^{1 / 2} \\
= & o(|\Lambda|)\left(\int_{Q^{\star}} \operatorname{Tr}_{L_{\xi}^{2}(Q)}\left[-\Delta_{\xi} K_{\xi}\right] \frac{d \xi}{(2 \pi)^{3}}\right)^{1 / 2},
\end{aligned}
$$

by a repeated use of the Cauchy-Schwarz inequality. Inserting (4.65), (4.66), (4.67) into (4.64), next in (4.63), we obtain

$$
\operatorname{Tr}_{L^{2}\left(\mathbf{R}^{3}\right)}\left[-\Delta K_{\Lambda}\right]=|\Lambda| \int_{Q^{\star}} \operatorname{Tr}_{L_{\xi}^{2}(Q)}\left[-\Delta_{\xi} K_{\xi}\right] \frac{d \xi}{(2 \pi)^{3}}+\mathrm{o}(|\Lambda|)
$$

which shows (4.62) and concludes the proof of the proposition.

\section{The Hartree-Fock model}

Let us first of all recall the Hartree-Fock model (2.3)-(2.4)-(2.5) introduced in Section 2:

$$
I_{\Lambda}^{H F}=\inf \left\{E_{\Lambda}^{H F}(K)+\frac{1}{2} U_{\Lambda} ; K \in \mathcal{K}_{\Lambda}\right\}
$$

where the set of minimization is

$$
\mathcal{K}_{\Lambda}=\left\{0 \leqslant K \leqslant \mathbf{1}, \operatorname{Tr} K=|\Lambda|, \operatorname{Tr}\left[\left(-\Delta-V_{\Lambda}\right) K\right]<+\infty\right\}
$$

and the energy functional writes

$$
\begin{aligned}
E_{\Lambda}^{H F}(K)= & \operatorname{Tr}\left[\left(-\Delta-V_{\Lambda}\right) K\right]+\frac{1}{2} \iint_{\mathbf{R}^{3} \times \mathbf{R}^{3}} \frac{\rho(x, x) \rho(y, y)}{|x-y|} d x d y \\
& -\frac{1}{2} \iint_{\mathbf{R}^{3} \times \mathbf{R}^{3}} \frac{|\rho(x, y)|^{2}}{|x-y|} d x d y,
\end{aligned}
$$

where $\rho(x ; y)$ is the Hilbert-Schmidt kernel of $K$. As is well-known, this functional is not convex, and therefore we expect the thermodynamic limit problem to be much more difficult than in the reduced Hartree-Fock setting. In this latter case, we have used in a fundamental way the convexity of the energy functional through the use of the $\sim$-transform. Here, for the Hartree-Fock case, this is not possible any more. Let us at once say that this is the main reason why we are not able to prove a result on 
the convergence of the Hartree-Fock energy per unit volume in the thermodynamic limit, and why we cannot establish the analogue of Theorem 2.2. Nevertheless, in this section, we shall (a) give some formal computations in order to justify our guess on the periodic Hartree-Fock problem (2.23)-(2.24)-(2.25) that should be obtained in the thermodynamic limit, (b) show that this periodic problem is well-posed mathematically (Theorem 2.3 in Subsection 5.1 below), and, finally, (c) check that the upper limit of the Hartree-Fock energy per unit volume may at least be compared from above by the HF periodic problem (Proposition 2.1 in Subsection 5.2).

Let us begin with some formal computations on the Hartree-Fock energy of a minimizer $K_{\Lambda}$ of $I_{\Lambda}^{H F}$ when $\Lambda$ goes to infinity.

We postulate that the sequence of operators $K_{\Lambda}$ converges to some self-adjoint operator $K$ that commutes with the translation of $\mathbf{Z}^{3}$, and that belongs to $\mathcal{K}$. By saying so, the main assumption we do is the following one: We postulate that the density $\rho_{\Lambda}(x, x)$ asymptotically behaves like a $Q$-periodic density $\rho(x, x)$. We emphasize this is an assumption, and that we only have the intuition that it is true. The lack of convexity of the Hartree-Fock model has prevented us so far to prove this postulate.

In view of the results we have obtained on the reduced Hartree-Fock model, it is then reasonable to believe that in the energy (2.5) of a minimizer $K_{\Lambda}$ of (2.3)-(2.4)-(2.5)

$$
\begin{aligned}
E_{\Lambda}^{H F}\left(K_{\Lambda}\right)= & \operatorname{Tr}\left[\left(-\Delta-V_{\Lambda}\right) K_{\Lambda}\right]+\frac{1}{2} \iint_{\mathbf{R}^{3} \times \mathbf{R}^{3}} \frac{\rho_{\Lambda}(x, x) \rho_{\Lambda}(y, y)}{|x-y|} d x d y \\
& -\frac{1}{2} \iint_{\mathbf{R}^{3} \times \mathbf{R}^{3}} \frac{\left|\rho_{\Lambda}(x, y)\right|^{2}}{|x-y|} d x d y,
\end{aligned}
$$

the first three terms globally behave like $|\Lambda|\left(E_{\mathrm{per}}^{R H F}(K)+\frac{M}{2}\right)$.

Therefore, it remains to understand, at least formally, the behaviour of the so-called exchange term $-\frac{1}{2} \iint_{\mathbf{R}^{3} \times \mathbf{R}^{3}} \frac{\left|\rho_{\Lambda}(x, y)\right|^{2}}{|x-y|} d x d y$. For this purpose, let us replace the density matrix $\rho_{\Lambda}(x, y)$ by a matrix of the form $\chi_{\Lambda}(x) \rho(x, y) \chi_{\Lambda}(y)$ mimicking the argument we have made above to determine the upper limit of the RHF model. The function $\chi_{\Lambda}$ is a cut-off function, which has all the good properties the reader may think of, and which are recalled in the proof of Proposition 2.1 below. Then, we establish, still in the course of Proposition 2.1 in Subsection 5.2 below, that

$$
\lim _{\Lambda \rightarrow \infty} \frac{1}{|\Lambda|} \iint_{\mathbf{R}^{3} \times \mathbf{R}^{3}} \frac{\left|\rho_{\Lambda}(x, y)\right|^{2}}{|x-y|} d x d y=\iint_{Q \times \mathbf{R}^{3}} \frac{|\rho(x, y)|^{2}}{|x-y|} d x d y .
$$

Moreover, the quantity which appears in the right-hand side of the above equality makes sense thanks to the following

LEMMA 5.1. - For any $K$ in $\mathcal{K}$, we have

$$
\int_{Q} d x \int_{\mathbf{R}^{3}} \frac{|\rho(x, y)|^{2}}{|x-y|} d y<+\infty
$$




$$
\begin{aligned}
& \int_{Q} d x \int_{\mathbf{R}^{3}} \frac{|\rho(x, y)|^{2}}{|x-y|} d y \\
& \quad=\iiint_{\left(Q^{\star}\right)^{2} \times Q^{2}} \rho(\xi, x, y) W_{\infty}\left(\xi-\xi^{\prime}, x-y\right) \rho^{*}\left(\xi^{\prime}, x, y\right) d x d y \frac{d \xi d \xi^{\prime}}{(2 \pi)^{6}}
\end{aligned}
$$

where the potential $W_{\infty}$ is given by (2.25) in Section 2; that is

$$
W_{\infty}(\eta, z)=\sum_{k \in \mathbf{Z}^{3}} \frac{\mathrm{e}^{i k \cdot \eta}}{|z+k|} .
$$

The proof of this lemma is given below.

The above argument justifies, at least formally, the introduction of the periodic problem (2.23)-(2.24)-(2.25), that we recall now:

$$
\begin{gathered}
I_{\mathrm{per}}^{H F}=\inf \left\{E_{\mathrm{per}}^{H F}(K) ; K \in \mathcal{K}\right\}, \\
E_{\mathrm{per}}^{H F}(K)=\int_{Q^{\star}} \operatorname{Tr}_{L_{\xi}^{2}(Q)}\left[-\Delta_{\xi} K_{\xi}\right] \frac{d \xi}{(2 \pi)^{3}}-\int_{Q} G \rho+\frac{1}{2} D_{G}(\rho, \rho)-\frac{1}{2} E_{\text {exc }}(K),
\end{gathered}
$$

where we denote by $E_{\text {exc }}(K)$ any of the two equivalent formulations (5.1a) and (5.1b) of the periodic exchange term. Before proving in the forthcoming subsection that the HF periodic problem is well-posed, we now give the proof of Lemma 5.1.

Proof of Lemma 5.1. - We split the exchange term into two terms in the following way

$$
\int_{Q} d x \int_{\mathbf{R}^{3}} \frac{|\rho(x, y)|^{2}}{|x-y|} d y=\int_{Q} d x \int_{|x-y| \leqslant 1} \frac{|\rho(x, y)|^{2}}{|x-y|} d y+\int_{Q} d x \int_{|x-y| \geqslant 1} \frac{|\rho(x, y)|^{2}}{|x-y|} d y .
$$

For the second term, we clearly have

$$
\int_{Q} d x \int_{|x-y| \geqslant 1} \frac{|\rho(x, y)|^{2}}{|x-y|} d y \leqslant \int_{Q} d x \int_{\mathbf{R}^{3}}|\rho(x, y)|^{2} d y<+\infty .
$$

Now, let us concentrate on the first term. Since we may prove like in (4.46) that $|\rho(x, y)|^{2} \leqslant \rho(x ; x) \rho(y ; y)$, almost everywhere on $\mathbf{R}^{3} \times \mathbf{R}^{3}$, we may write

$$
\begin{aligned}
\int_{Q} d x \int_{|x-y| \leqslant 1} \frac{|\rho(x, y)|^{2}}{|x-y|} d y & \leqslant \int_{Q} d x \int_{|x-y| \leqslant 1} \frac{\rho(x ; x) \rho(y ; y)}{|x-y|} d y \\
& \leqslant \iint_{\left(Q+B_{1}\right) \times\left(Q+B_{1}\right)} \frac{\rho(x ; x) \rho(y ; y)}{|x-y|} d x d y \\
& \leqslant C\|\rho\|_{L^{6 / 5}\left(Q+B_{1}\right)}^{2} \\
& \leqslant C\|\rho\|_{L_{\text {unif }}^{6 / 5}\left(\mathbf{R}^{3}\right)}<+\infty
\end{aligned}
$$


since $\rho$ lies in $H_{\text {unif }}^{1}\left(\mathbf{R}^{3}\right)$. We now check the equivalence of the two formulations (5.1a) and $(5.1 \mathrm{~b})$, which follows from the following string of equalities:

$$
\begin{aligned}
& \iint_{Q \times \mathbf{R}^{3}} \frac{|\rho(x, y)|^{2}}{|x-y|} d x d y \\
& \quad=\iint_{Q \times \mathbf{R}^{3}} \frac{1}{|x-y|} d x d y \iint_{Q^{\star} \times Q^{\star}} \rho(\xi, x, y) \rho^{*}\left(\xi^{\prime}, x, y\right) \frac{d \xi d \xi^{\prime}}{(2 \pi)^{6}} \\
& \quad=\iiint \int_{Q^{2} \times\left(Q^{\star}\right)^{2}} \sum_{k \in \mathbf{Z}^{3}} \frac{1}{|x-y-k|} \rho(\xi, x, y+k) \rho^{*}\left(\xi^{\prime}, x, y+k\right) d x d y \frac{d \xi d \xi^{\prime}}{(2 \pi)^{6}} \\
& =\iiint_{Q^{2} \times\left(Q^{\star}\right)^{2}} \rho(\xi, x, y) \rho^{*}\left(\xi^{\prime}, x, y\right) \sum_{k \in \mathbf{Z}^{3}} \frac{\mathrm{e}^{-i\left(\xi-\xi^{\prime}\right) \cdot k}}{|x-y-k|} d x d y \frac{d \xi d \xi^{\prime}}{(2 \pi)^{6}} \\
& =\iiint_{Q^{2} \times\left(Q^{\star}\right)^{2}} \rho(\xi, x, y) W^{\infty}\left(\xi-\xi^{\prime}, x-y\right) \rho^{*}\left(\xi^{\prime}, x, y\right) d x d y \frac{d \xi d \xi^{\prime}}{(2 \pi)^{6}},
\end{aligned}
$$

where we have used the properties of periodicity of the functions $\rho(\xi, x, y)$ with respect to $y$, and the definition (2.25) of $W^{\infty}$.

The proof of Lemma 5.1 is complete.

This section is organized as follows. We first prove in Subsection 5.1 that the periodic HF problem is well-posed. Next, in Subsection 5.2, we check that we may compare from above the Hartree-Fock energy per unit volume with this periodic problem, by using a minimizer of $I_{\text {per }}^{H F}$.

\subsection{The periodic HF problem}

This section is devoted to the proof of Theorem 2.3, that we recall here for the convenience of the reader.

THEOREM 2.3 (Well-posedness of the HF periodic problem). - The minimization problem defined by (2.23) and (2.24) (respectively by (2.26) and (2.27)) admits a minimum.

We shall provide two different proofs of this claim. The first one makes use of regularity properties of the potential $W(\eta, z)$ given by (2.25), and which appears when one writes the exchange terms according to the Bloch waves decomposition. The second one, which is also the shortest one, is based upon the formulation (5.1a) of the exchange term.

First proof of Theorem 2.3. - In order to check that the minimization problem (2.23)(2.24)-(2.25) is well-posed, we now prove that, given an arbitrary sequence of operators $K_{n}(\in \mathcal{K})$ such that $E_{\text {per }}^{H F}\left(K_{n}\right)$ goes to $I_{\text {per }}^{H F}$, as $n$ goes to infinity, this sequence converges, up to an extraction of a subsequence, to some operator $K(\in \mathcal{K})$ that satisfies $E_{\text {per }}^{H F}(K)=$ $I_{\text {per }}^{H F}$. For this purpose, we shall heavily rely upon the proof of Theorem 2.1. But, first 
of all, we begin our proof with a careful study of the properties of regularity of the interaction potential $W_{\infty}$ and consequently of the properties of the exchange term.

Step 1. - Decomposition of the exchange potential.

We first remark that the function $\mathrm{e}^{i \eta \cdot x} W_{\infty}(\eta, x)$ is $Q$-periodic with respect to $x$ when $\eta$ is fixed. Indeed, $j \in \mathbf{Z}^{3}$ being fixed, we have

$$
\begin{aligned}
\mathrm{e}^{i \eta \cdot(x+j)} W_{\infty}(\eta, x+j) & =\mathrm{e}^{i \eta \cdot x} \mathrm{e}^{i \eta \cdot j} \sum_{k \in \mathbf{Z}^{3}} \frac{\mathrm{e}^{i k \cdot \eta}}{|x+j+k|} \\
& =\mathrm{e}^{i \eta \cdot x} \sum_{m \in \mathbf{Z}^{3}} \frac{\mathrm{e}^{i m \cdot \eta}}{|x+m|}=\mathrm{e}^{i \eta \cdot x} W_{\infty}(\eta, x),
\end{aligned}
$$

for almost all $(\eta, x) \in Q^{\star} \times Q$. We may therefore decompose $\mathrm{e}^{i \eta \cdot x} W_{\infty}(\eta$, $x$ ) into its Fourier series:

$$
\mathrm{e}^{i \eta \cdot x} W_{\infty}(\eta, x)=\sum_{m \in \mathbf{Z}^{3}} a_{m}(\eta) \mathrm{e}^{2 i \pi m \cdot x}
$$

with coefficients $a_{m}(\eta)$ given by

$$
\begin{aligned}
a_{m}(\eta) & =\int_{Q} \mathrm{e}^{i \eta \cdot x} W_{\infty}(\eta, x) \mathrm{e}^{-2 i \pi m \cdot x} d x \\
& =\int_{Q} \mathrm{e}^{i(\eta-2 \pi m) \cdot x} \sum_{k \in \mathbf{Z}^{3}} \frac{\mathrm{e}^{i k \cdot \eta}}{|x+k|} d x \\
& =\sum_{k \in \mathbf{Z}^{3}} \int_{Q} \mathrm{e}^{i(\eta-2 \pi m) \cdot(x+k)} \frac{1}{|x+k|} d x \\
& =\int_{\mathbf{R}^{3}} \mathrm{e}^{i(\eta-2 \pi m) \cdot y} \frac{1}{|y|} d y=\frac{4 \pi}{|\eta-2 \pi m|^{2}},
\end{aligned}
$$

for almost all $\eta \in Q^{\star}$. Hence, we have

$$
W_{\infty}(\eta, x)=4 \pi \mathrm{e}^{-i \eta \cdot x} \sum_{m \in \mathbf{Z}^{3}} \frac{\mathrm{e}^{2 i \pi m \cdot x}}{|\eta-2 \pi m|^{2}} .
$$

It is easily deduced from this expression that, with $x$ being fixed in $Q$, the function defined by $W_{\infty}(\eta, x)-4 \pi \frac{\mathrm{e}^{-i \eta \cdot x}}{|\eta|^{2}}$ is continuous with respect to $\eta \in Q^{\star}$, and even to $\eta \in(1+\varepsilon) Q(\varepsilon>0$, small enough), and satisfies

$$
\lim _{\eta \rightarrow 0}\left(W_{\infty}(\eta, x)-4 \pi \frac{\mathrm{e}^{-i \eta \cdot x}}{|\eta|^{2}}\right)=\sum_{m \in \mathbf{Z}^{3} \backslash\{0\}} \frac{\mathrm{e}^{2 i \pi m \cdot x}}{\pi|m|^{2}}=G(x) .
$$


Since we have isolated the singularity in $\eta$, let us now examine the singularity in $x$. Let us then consider

$$
f(\eta, x)=W_{\infty}(\eta, x)-\mathrm{e}^{-i \eta \cdot x} G(x)-4 \pi \frac{\mathrm{e}^{-i \eta \cdot x}}{|\eta|^{2}} .
$$

We now check that $f(\eta, x)$ is in $L^{\infty}\left(Q^{\star} \times Q\right)$, and even in $L^{\infty}\left((1+\varepsilon) Q^{\star} \times(1+\varepsilon) Q\right)$. From (5.2) and the Fourier series expansion of $G$, we obtain

$$
\begin{aligned}
f(\eta, x) & =4 \pi \mathrm{e}^{-i \eta \cdot x} \sum_{m \in \mathbf{Z}^{3} \backslash\{0\}} \mathrm{e}^{2 i \pi m \cdot x}\left(\frac{1}{|\eta-2 \pi m|^{2}}-\frac{1}{|2 \pi m|^{2}}\right) \\
& =4 \pi \mathrm{e}^{-i \eta \cdot x} \sum_{m \in \mathbf{Z}^{3} \backslash\{0\}} \mathrm{e}^{2 i \pi m \cdot x} \frac{4 \pi \eta \cdot m-|\eta|^{2}}{|\eta-2 \pi m|^{2}|2 \pi m|^{2}} .
\end{aligned}
$$

It is obvious from the last equality that, at fixed $\eta, \mathrm{e}^{i \eta \cdot x} f(\eta, x)$ is $Q$-periodic with respect to $x$, and that

$$
\|f(\eta, \cdot)\|_{L^{2}(Q)}^{2} \leqslant C \sum_{m \in \mathbf{Z}^{3} \backslash\{0\}} \frac{1+|m|^{2}}{|\eta-2 \pi m|^{4}|2 \pi m|^{4}},
$$

for some positive constant which is independent of $\eta \in(1+\varepsilon) Q^{\star}$; in other words, for any $\varepsilon>0$ small enough,

$$
\|f(\eta, \cdot)\|_{L^{2}(Q)}^{2} \in L^{\infty}\left((1+\varepsilon) Q^{\star}\right) .
$$

With $\eta$ being still fixed in $(1+\varepsilon) Q^{\star}$, it is clear from (5.3) that

$$
-\Delta_{x} f(\eta, x)=4 \pi \sum_{k \in \mathbf{Z}^{3} \backslash\{0\}}\left(\mathrm{e}^{-i k \cdot \eta}-1\right) \delta_{k}(x),
$$

and thus $f(\eta, x)$ is harmonic in $(1+\varepsilon) Q$. With the help of the mean-value property, we finally obtain for every $x$ in $Q$,

$$
|f(\eta, x)| \leqslant \int_{x+\varepsilon Q}|f(\eta, y)| d y \leqslant C\|f(\eta, \cdot)\|_{L^{2}(Q)}^{1 / 2},
$$

and we conclude since the right-hand side in the above inequality lies in $L^{\infty}\left(Q^{\star}\right)$ thanks to (5.5).

Next, we remark that the exchange term involves the function $W_{\infty}\left(\xi-\xi^{\prime}, x-\right.$ $y$ ) with $\xi$ and $\xi^{\prime}$ varying in $Q^{\star}$, and $x$ and $y$ varying in $Q$. Therefore, we need some information on $W_{\infty}(\eta, x)$ on $2 Q^{\star} \times 2 Q=\left[-2 \pi,+2 \pi\left[{ }^{3} \times\left[-1,+1\left[^{3}\right.\right.\right.\right.$. In a straightforward way, for almost all $\eta \in\left[-2 \pi,+2 \pi\left[^{3}, x \in\left[-1,+1\left[^{3}\right.\right.\right.\right.$, we obtain from (5.3) the decomposition

$$
W_{\infty}(\eta, x)=\bar{W}_{\infty}(\eta, x)-\sum_{k \in \mathbf{Z}^{3},|k|_{\infty} \leqslant 1} \frac{1}{|x+k|}
$$




$$
-4 \pi \mathrm{e}^{-i \eta \cdot x} \sum_{m \in \mathbf{Z}^{3},|m|_{\infty} \leqslant 1} \frac{\mathrm{e}^{2 i \pi m \cdot x}}{|\eta-2 \pi m|^{2}},
$$

with $|x|_{\infty}=\max \left(\left|x_{1}\right|,\left|x_{2}\right|,\left|x_{3}\right|\right)$, and where $\bar{W}_{\infty}(\eta, x)$ belongs to $L^{\infty}\left(2 Q^{\star} \times 2 Q\right)$, owing in particular to the fact that $G(x)-\sum_{k \in \mathbf{Z}^{3},|k|_{\infty} \leqslant 1} \frac{1}{|x+k|}$ is bounded in $2 Q^{\star} \times 2 Q$.

Step 2. - A priori estimates.

Using the decomposition (5.6), let us now show that the a priori estimates that have been established in Step 1 of the proof of Theorem 2.1 also hold true here.

Since some estimates only depend on the constraints and not on the energy functional, it is easy to see that the following bounds of the RHF setting are also valid here:

$$
\left\{\begin{array}{l}
\rho_{n}(\xi, x, y) \text { is bounded in } L^{2}\left(Q^{\star} \times Q \times Q\right), \\
\rho_{n}(x, y) \text { is bounded in } L^{2}\left(Q \times \mathbf{R}^{3}\right), \\
\rho_{n}(x, x) \text { is bounded in } L^{1}(Q) .
\end{array}\right.
$$

Next, we are going to estimate the exchange term by splitting it into three terms according to the decomposition (5.6). First, we have

$$
\begin{aligned}
& \left|\iiint_{Q^{2} \times\left(Q^{\star}\right)^{2}} \rho_{n}(\xi, x, y) \overline{W_{\infty}}\left(\xi-\xi^{\prime}, x-y\right) \rho_{n}^{*}\left(\xi^{\prime}, x, y\right) d x d y d \xi d \xi^{\prime}\right| \\
& \quad \leqslant\left\|\overline{W_{\infty}}\right\|_{L^{\infty}} \iiint \int_{Q^{2} \times\left(Q^{\star}\right)^{2}}\left|\rho_{n}(\xi, x, y)\right|\left|\rho_{n}^{*}\left(\xi^{\prime}, x, y\right)\right| d x d y d \xi d \xi^{\prime} \\
& \quad \leqslant\left\|\overline{W_{\infty}}\right\|_{L^{\infty}}\left|Q^{\star}\right| \iiint_{Q^{2} \times Q^{\star}}\left|\rho_{n}(\xi, x, y)\right|^{2} d x d y d \xi \leqslant C,
\end{aligned}
$$

by the Cauchy-Schwarz inequality and (5.7), where $C$ denotes here and below various positive constants that are independent of $n$.

Next, we treat for instance the term:

$$
\begin{aligned}
& \left|\iiint \int_{Q^{2} \times\left(Q^{\star}\right)^{2}} \rho_{n}(\xi, x, y) \frac{1}{|x-y|} \rho_{n}^{*}\left(\xi^{\prime}, x, y\right) \frac{d \xi d \xi^{\prime}}{(2 \pi)^{6}} d x d y\right| \\
& \quad=\iint_{Q \times Q} \frac{\left|\rho_{n}(x, y)\right|^{2}}{|x-y|} d x d y \leqslant \iint_{Q \times Q} \frac{\rho_{n}(x, x) \rho_{n}(y, y)}{|x-y|} d x d y,
\end{aligned}
$$

since we recall that, by the discrete and next the continuous Cauchy-Schwarz inequality, we have

$$
\begin{aligned}
\left|\rho_{n}(x, y)\right| & =\left|\int_{Q^{\star}} \sum_{n \geqslant 1} \lambda_{n}(\xi) u_{n}(\xi, x) u_{n}^{*}(\xi, y) \frac{d \xi}{(2 \pi)^{3}}\right| \\
& \leqslant \int_{Q^{\star}}\left(\sum_{n \geqslant 1} \lambda_{n}(\xi)\left|u_{n}(\xi, x)\right|^{2}\right)^{1 / 2}\left(\sum_{n \geqslant 1} \lambda_{n}(\xi)\left|u_{n}(\xi, y)\right|^{2}\right)^{1 / 2} \frac{d \xi}{(2 \pi)^{3}}
\end{aligned}
$$




$$
\begin{aligned}
& \leqslant\left(\int_{Q^{\star}} \sum_{n \geqslant 1} \lambda_{n}(\xi)\left|u_{n}(\xi, x)\right|^{2} \frac{d \xi}{(2 \pi)^{3}}\right)^{1 / 2}\left(\int_{Q^{\star}} \sum_{n \geqslant 1} \lambda_{n}(\xi)\left|u_{n}(\xi, y)\right|^{2} \frac{d \xi}{(2 \pi)^{3}}\right)^{1 / 2} \\
& \leqslant \sqrt{\rho_{n}(x, x)} \sqrt{\rho_{n}(y, y)} .
\end{aligned}
$$

Thus, we have in (5.9)

$$
\begin{aligned}
& \left|\iiint_{Q^{2} \times\left(Q^{\star}\right)^{2}} \rho_{n}(\xi, x, y) \frac{1}{|x-y|} \rho_{n}^{*}\left(\xi^{\prime}, x, y\right) d x d y \frac{d \xi d \xi^{\prime}}{(2 \pi)^{6}}\right| \\
& \quad \leqslant \int_{Q} \rho_{n}(x, x)\left(\rho_{n}(\cdot, \cdot) \chi_{Q} \star \frac{1}{|x|}\right) d x \\
& \quad \leqslant C\left\|\frac{1}{|x|}\right\|_{L^{3, \infty}}\left\|\rho_{n}(x, x)\right\|_{L^{6 / 5}(Q)}^{2} \\
& \quad \leqslant C\left\|\frac{1}{|x|}\right\|_{L^{3, \infty}}\left\|\rho_{n}(x, x)\right\|_{L^{1}(Q)}^{3 / 2}\left\|\rho_{n}(x, x)\right\|_{L^{3}(Q)}^{1 / 2}
\end{aligned}
$$

using Young's and then Hölder's inequality. Next, using the Sobolev embeddings and (5.7), we obtain

$$
\begin{aligned}
& \left|\iiint \int_{Q^{2} \times\left(Q^{\star}\right)^{2}} \rho_{n}(\xi, x, y) \frac{1}{|x-y|} \rho_{n}^{*}\left(\xi^{\prime}, x, y\right) \frac{d \xi d \xi^{\prime}}{(2 \pi)^{6}} d x d y\right| \\
& \quad \leqslant C\left\|\frac{1}{|x|}\right\|_{L^{3, \infty}}\left\|\rho_{n}(x, x)\right\|_{L^{1}(Q)}^{3 / 2}\left\|\sqrt{\rho_{n}(x, x)}\right\|_{H^{1}(Q)} \\
& \quad \leqslant C\left\|\frac{1}{|x|}\right\|_{L^{3, \infty}}\left\|\rho_{n}(x, x)\right\|_{L^{1}(Q)}^{3 / 2}\left(1+\left\|\nabla \sqrt{\rho_{n}(x, x)}\right\|_{L^{2}(Q)}\right)^{1 / 2} .
\end{aligned}
$$

Finally, we treat the contribution of the third term in (5.6), namely

$$
\begin{aligned}
& \left|\iiint_{Q^{2} \times\left(Q^{\star}\right)^{2}} \rho_{n}(\xi, x, y) \frac{\mathrm{e}^{-i\left(\xi-\xi^{\prime}\right) \cdot(x-y)}}{\left|\xi-\xi^{\prime}\right|^{2}} \rho_{n}^{*}\left(\xi^{\prime}, x, y\right) \frac{d \xi d \xi^{\prime}}{(2 \pi)^{6}} d x d y\right| \\
& \quad \leqslant \iint_{Q \times Q}\left\|\left(\left|\rho_{n}(\cdot, x, y)\right| \star Q^{\star} \frac{1}{|\xi|^{2}}\right)\left|\rho_{n}(\cdot, x, y)\right|\right\|_{L^{1}\left(Q^{\star}\right)} d x d y,
\end{aligned}
$$

where, here and below, we shall use the notation $f \star Q^{\star} g(\xi)=\int_{Q^{\star}} f\left(\xi-\xi^{\prime}\right) g\left(\xi^{\prime}\right) \frac{d \xi^{\prime}}{(2 \pi)^{3}}$. By the Young and the Hölder inequalities, we have, with $x$ and $y$ being fixed,

$$
\begin{aligned}
& \left\|\left(\left|\rho_{n}(\cdot, x, y)\right| \star_{Q^{\star}} \frac{1}{|\xi|^{2}}\right)\left|\rho_{n}(\cdot, x, y)\right|\right\|_{L^{1}\left(Q^{\star}\right)} \\
& \quad \leqslant\left\|\left|\rho_{n}(\cdot, x, y)\right| \star_{Q^{\star}} \frac{1}{|\xi|^{2}}\right\|_{L^{2}\left(Q^{\star}\right)}\left\|\rho_{n}(\cdot, x, y)\right\|_{L^{2}\left(Q^{\star}\right)} \\
& \quad \leqslant\left\|\frac{1}{|\xi|^{2}}\right\|_{L^{1}\left(Q^{\star}\right)}\left\|\rho_{n}(\cdot, x, y)\right\|_{L^{2}\left(Q^{\star}\right)}^{2} .
\end{aligned}
$$

Therefore, 


$$
\begin{aligned}
& \left|\iiint \int_{Q^{2} \times\left(Q^{\star}\right)^{2}} \rho_{n}(\xi, x, y) \frac{\mathrm{e}^{-i\left(\xi-\xi^{\prime}\right) \cdot(x-y)}}{\left|\xi-\xi^{\prime}\right|^{2}} \rho_{n}^{*}\left(\xi^{\prime}, x, y\right) \frac{d \xi d \xi^{\prime}}{(2 \pi)^{6}} d x d y\right| \\
& \quad \leqslant\left\|\frac{1}{|\xi|^{2}}\right\|_{L^{1}\left(Q^{\star}\right)} \iint_{Q \times Q}\left\|\rho_{n}(\cdot, x, y)\right\|_{L^{2}\left(Q^{\star}\right)}^{2} d x d y \\
& \quad=\left\|\frac{1}{|\xi|^{2}}\right\|_{L^{1}\left(Q^{\star}\right)}\left\|\rho_{n}(\xi, x, y)\right\|_{L^{2}\left(Q^{\star} \times Q^{2}\right)}^{2} \leqslant C,
\end{aligned}
$$

in view of (5.7). We now collect (5.8), (5.10), and (5.11), and estimate the exchange term as follows

$$
\begin{aligned}
& \left|\iiint_{Q^{2} \times\left(Q^{\star}\right)^{2}} \rho_{n}(\xi, x, y) W_{\infty}\left(\xi-\xi^{\prime}, x-y\right) \rho_{n}^{*}\left(\xi^{\prime}, x, y\right) \frac{d \xi d \xi^{\prime}}{(2 \pi)^{6}} d x d y\right| \\
& \quad \leqslant C+C\left\|\nabla \sqrt{\rho_{n}(x, x)}\right\|_{L^{2}(Q)^{\prime}} .
\end{aligned}
$$

It now remains to copy the proof of Theorem 2.1: The kinetic energy term is bounded from below by $\left\|\nabla \sqrt{\rho_{n}(x, x)}\right\|_{L^{2}(Q)}^{2}$, and therefore the fact that the energy of the minimizing sequence converges to the infimum implies that $\left\|\nabla \sqrt{\rho_{n}(x, x)}\right\|_{L^{2}(Q)}$ is bounded. Consequently, all the bounds shown in the RHF setting still hold here:

$$
\left\{\begin{array}{l}
\sqrt{\rho_{n}(x, x)} \text { is bounded in } H^{1}(Q), \text { thus in } L^{p}(Q), 1 \leqslant p \leqslant 6, \\
\rho_{n}(x, y) \text { is bounded in } L^{p}(Q \times Q), 1 \leqslant p \leqslant 6, \\
\sqrt{\rho_{n}(\xi, x, x)} \text { is bounded in } L^{2}\left(Q^{\star} ; H^{1}(Q)\right), \\
\rho_{n}(\xi, x, x) \text { is bounded in } L^{5 / 3}\left(Q^{\star} \times Q\right),
\end{array}\right.
$$

and each of the four terms of the energy (2.24) is bounded independently of $n$. As a consequence of (5.13), we show the following bound that will be useful in Step 3:

$$
\rho_{n}(x, y) \text { is bounded in } H^{1}(Q \times Q) .
$$

Indeed, we have

$$
\begin{aligned}
\left|\nabla_{x} \rho_{n}(x, y)\right|= & \left|\int_{Q^{\star}} \sum_{m \geqslant 1} \lambda_{m}(\xi) \nabla_{x} u_{m}(\xi, x) u_{m}^{*}(\xi, y) \frac{d \xi}{(2 \pi)^{3}}\right| \\
\leqslant & \int_{Q^{\star}}\left(\sum_{m \geqslant 1} \lambda_{m}(\xi)\left|\nabla_{x} u_{m}(\xi, x)\right|^{2}\right)^{1 / 2}\left(\sum_{m \geqslant 1} \lambda_{m}(\xi)\left|u_{m}(\xi, y)\right|^{2}\right)^{1 / 2} \frac{d \xi}{(2 \pi)^{3}} \\
\leqslant & \left(\int_{Q^{\star}} \sum_{m \geqslant 1} \lambda_{m}(\xi)\left|\nabla_{x} u_{m}(\xi, x)\right|^{2} \frac{d \xi}{(2 \pi)^{3}}\right)^{1 / 2} \\
& \times\left(\int_{Q^{\star}} \sum_{m \geqslant 1} \lambda_{m}(\xi)\left|u_{m}(\xi, y)\right|^{2} \frac{d \xi}{(2 \pi)^{3}}\right)^{1 / 2} .
\end{aligned}
$$

Hence, 


$$
\begin{aligned}
& \iint_{Q \times Q}\left|\nabla_{x} \rho_{n}(x, y)\right|^{2} d x d y \\
& \quad \leqslant \iint_{Q \times Q^{\star}} \sum_{m \geqslant 1} \lambda_{m}(\xi)\left|\nabla_{x} u_{m}(\xi, x)\right|^{2} \frac{d x d \xi}{(2 \pi)^{3}} \iint_{Q \times Q^{\star}} \sum_{m \geqslant 1} \lambda_{m}(\xi)\left|u_{m}(\xi, y)\right|^{2} \frac{d y d \xi}{(2 \pi)^{3}} \\
& \quad=\int_{Q^{\star}} \operatorname{Tr}_{L_{\xi}^{2}(Q)}\left[-\Delta_{\xi} K_{\xi}\right] \frac{d \xi}{(2 \pi)^{3}}\left\|\rho_{n}(x, x)\right\|_{L^{1}(Q)} \leqslant C .
\end{aligned}
$$

This yields (5.14).

Step 3. - Convergence of the exchange term.

In view of the bounds (5.7), (5.13), (5.14), we may choose a subsequence of $K_{n}$, still denoted by $K_{n}$, such that the following convergences hold:

$$
\left\{\begin{array}{l}
\rho_{n}(\xi, x, y) \text { converges weakly in } L^{2}\left(Q^{\star} \times Q \times Q\right), \\
\rho_{n}(\xi, x, x) \text { converges weakly in } L^{5 / 3}\left(Q^{\star} \times Q\right), \\
\rho_{n}(x, y) \text { converges strongly in } L^{p}(Q \times Q), 1 \leqslant p<6 .
\end{array}\right.
$$

The last convergence holds, because by Rellich's theorem, (5.14) implies that $\rho_{n}(x, y)$ strongly converges (up to the extraction of a subsequence) in $L^{p}\left(Q^{2}\right)$ for all $1 \leqslant p<3$. But, since we have in addition the second bound of (5.7), the interpolation inequality yields the strong convergence in $L^{p}\left(Q^{2}\right)$ for all $1 \leqslant p<6$. Let us denote by $K=$ $\int_{Q^{\star}} K_{\xi} \frac{d \xi}{(2 \pi)^{3}}$ the operator that is the limit of $K_{n}$ in the sense of the operators weak topology, and in the sense of (4.50) - the latter being equivalent to the first convergence of (5.15). Let $\rho(\xi, x, y)$ denotes the kernel of the associated $K_{\xi}$ : It is indeed clear that $K \in \mathcal{K}$ for the same reasons as those indicated in the proof of Theorem 2.1. In particular, $K$ still satisfies the constraint of charge 1 . Likewise, we have

$$
\liminf _{n \rightarrow+\infty} \int_{Q^{\star}} \operatorname{Tr}_{L_{\xi}^{2}(Q)}\left[-\Delta_{\xi} K_{\xi}^{n}\right] \frac{d \xi}{(2 \pi)^{3}} \geqslant \int_{Q^{\star}} \operatorname{Tr}_{L_{\xi}^{2}(Q)}\left[-\Delta_{\xi} K_{\xi}\right] \frac{d \xi}{(2 \pi)^{3}},
$$

and the electrostatic terms converge as they do in the RHF setting. Concluding the proof of Theorem 2.3 therefore amounts to proving that we may pass to the limit in the exchange term, or in other words that

$$
\begin{gathered}
\lim _{n \rightarrow+\infty} \iiint \int_{Q^{2} \times\left(Q^{\star}\right)^{2}} \rho_{n}(\xi, x, y) W_{\infty}\left(\xi-\xi^{\prime}, x-y\right) \rho_{n}^{*}\left(\xi^{\prime}, x, y\right) d \xi d \xi^{\prime} d x d y \\
\quad=\iiint \int_{Q^{2} \times\left(Q^{\star}\right)^{2}} \rho(\xi, x, y) W_{\infty}\left(\xi-\xi^{\prime}, x-y\right) \rho^{*}\left(\xi^{\prime}, x, y\right) d \xi d \xi^{\prime} d x d y .
\end{gathered}
$$

In order to prove (5.16), we again decompose the interaction potential $W_{\infty}(\cdot, \cdot)$ accordingly to (5.6). We treat each of the three categories of terms separately, proving the analogue of (5.16) for each of them. The assertion (5.16) will then follow by addition.

We begin with proving 


$$
\begin{gathered}
\lim _{n \rightarrow+\infty} \iiint_{Q^{2} \times\left(Q^{\star}\right)^{2}} \rho_{n}(\xi, x, y) \overline{W_{\infty}}\left(\xi-\xi^{\prime}, x-y\right) \rho_{n}^{*}\left(\xi^{\prime}, x, y\right) d \xi d \xi^{\prime} d x d y \\
=\iiint \int_{Q^{2} \times\left(Q^{\star}\right)^{2}} \rho(\xi, x, y) \overline{W_{\infty}}\left(\xi-\xi^{\prime}, x-y\right) \rho^{*}\left(\xi^{\prime}, x, y\right) d \xi d \xi^{\prime} d x d y
\end{gathered}
$$

Clearly, it suffices to prove

$$
\begin{aligned}
& \lim _{n \rightarrow+\infty} \iiint_{Q^{2} \times\left(Q^{\star}\right)^{2}}\left[\rho_{n}(\xi, x, y)-\rho(\xi, x, y)\right] \overline{W_{\infty}}\left(\xi-\xi^{\prime}, x-y\right) \\
& \quad \times \rho_{n}^{*}\left(\xi^{\prime}, x, y\right) d \xi d \xi^{\prime} d x d y=0 .
\end{aligned}
$$

For this purpose, we observe that

$$
\begin{aligned}
& \left|\iiint_{Q^{2} \times\left(Q^{\star}\right)^{2}}\left[\rho_{n}(\xi, x, y)-\rho(\xi, x, y)\right] \overline{W_{\infty}}\left(\xi-\xi^{\prime}, x-y\right) \rho_{n}^{*}\left(\xi^{\prime}, x, y\right) \frac{d \xi d \xi^{\prime}}{(2 \pi)^{6}} d x d y\right| \\
& \quad \leqslant \iint_{Q \times Q}\left|\int_{Q^{\star}} \rho_{n}(\xi, x, y) \frac{d \xi}{(2 \pi)^{3}}-\iint_{Q^{\star}} \rho(\xi, x, y) \frac{d \xi}{(2 \pi)^{3}}\right|\left|\overline{W_{\infty}}\left(\xi-\xi^{\prime}, x-y\right)\right| \\
& \quad \times\left|\int_{Q^{\star}} \rho_{n}^{*}\left(\xi^{\prime}, x, y\right) \frac{d \xi^{\prime}}{(2 \pi)^{3}}\right| d x d y \\
& \leqslant\left\|\overline{W_{\infty}}\right\|_{L^{\infty}} \iint_{Q \times Q}\left|\rho_{n}(x, y)-\rho(x, y)\right|\left|\rho_{n}(x, y)\right| d x d y \\
& \leqslant\left\|\overline{W_{\infty}}\right\|_{L^{\infty}}\left\|\rho_{n}(x, y)-\rho(x, y)\right\|_{L^{2}\left(Q^{2}\right)}\left\|\rho_{n}(x, y)\right\|_{L^{2}\left(Q^{2}\right)},
\end{aligned}
$$

and (5.17) follows from the strong convergence of $\rho_{n}(x, y)$ to $\rho(x, y)$ in $L^{2}\left(Q^{2}\right)$.

For the second term, we remark that

$$
\begin{aligned}
& \left|\iiint \int_{Q^{2} \times\left(Q^{\star}\right)^{2}}\left[\left|\rho_{n}(x, y)\right|^{2}-|\rho(x, y)|^{2}\right] \frac{1}{|x-y|} d x d y\right| \\
& \quad \leqslant C\left\|\frac{1}{|x-y|}\right\|_{L^{2}\left(Q^{2}\right)}\left\|\left|\rho_{n}(x, y)\right|^{2}-|\rho(x, y)|^{2}\right\|_{L^{2}\left(Q^{2}\right)},
\end{aligned}
$$

where the right-hand side converges to zero because $\rho_{n}(x, y)$ converges to $\rho(x, y)$, strongly in $L^{4}\left(Q^{2}\right)$, thus $\left|\rho_{n}(x, y)\right|^{2}$ converges to $|\rho(x, y)|^{2}$, strongly in $L^{2}\left(Q^{2}\right)$.

It now remains to treat the third term of (5.6), namely, for instance, to prove

$$
\begin{aligned}
& \lim _{n \rightarrow+\infty} \iiint \int_{Q^{2} \times\left(Q^{\star}\right)^{2}} \rho_{n}(\xi, x, y) \rho_{n}^{*}\left(\xi^{\prime}, x, y\right) \frac{\mathrm{e}^{-i\left(\xi-\xi^{\prime}\right) \cdot(x-y)}}{\left|\xi-\xi^{\prime}\right|^{2}} d \xi d \xi^{\prime} d x d y \\
& \quad=\iiint \int_{Q^{2} \times\left(Q^{\star}\right)^{2}} \rho(\xi, x, y) \rho^{*}\left(\xi^{\prime}, x, y\right) \frac{\mathrm{e}^{-i\left(\xi-\xi^{\prime}\right) \cdot(x-y)}}{\left|\xi-\xi^{\prime}\right|^{2}} d \xi d \xi^{\prime} d x d y .
\end{aligned}
$$


We introduce the function

$$
F_{n}(\xi, x, y)=\int_{Q^{\star}} \rho_{n}^{*}\left(\xi^{\prime}, x, y\right) \frac{\mathrm{e}^{-i\left(\xi-\xi^{\prime}\right) \cdot(x-y)}}{\left|\xi-\xi^{\prime}\right|^{2}} \frac{d \xi^{\prime}}{(2 \pi)^{3}},
$$

and the analogous function $F$ when $\rho_{n}$ is replaced by $\rho$. What we have to prove is that

$$
\begin{aligned}
& \lim _{n \rightarrow+\infty} \iiint_{Q^{2} \times Q^{\star}}\left[\rho_{n}(\xi, x, y) F_{n}(\xi, x, y)\right. \\
& \quad-\rho(\xi, x, y) F(\xi, x, y)] d \xi d x d y=0,
\end{aligned}
$$

and for this purpose it suffices to show that $\rho_{n}(\xi, x, y)$ converges weakly to $\rho(\xi, x, y)$ in $L^{2}\left(Q^{\star} \times Q^{2}\right)$, which we already know from (5.15), and that $F_{n}(\xi, x, y)$ converges strongly to $F(\xi, x, y)$ in $L^{2}\left(Q^{\star} \times Q^{2}\right)$, which we now establish (up to the extraction of a subsequence).

Since $\frac{\mathrm{e}^{-i \xi \cdot(x-y)}}{|\xi|^{2}}$ belongs to $L^{\infty}\left(Q \times Q ; L^{1}\left(Q^{\star}\right)\right)$, and since $\rho_{n}(\xi, x, y)$ is bounded in $L^{2}\left(Q^{\star} \times Q^{2}\right)$, we easily deduce from Young's inequality that

$$
F_{n}(\xi, x, y) \text { is bounded in } L^{2}\left(Q^{\star} \times Q \times Q\right) .
$$

Likewise, the generalized Young inequality for the Marcinkiewicz spaces (or weak $L^{p}$ spaces) implies

$$
F_{n}(\xi, x, y) \text { is bounded in } L^{6}\left(Q^{\star} \times Q \times Q\right),
$$

using this time that $\frac{\mathrm{e}^{-i \xi \cdot(x-y)}}{|\xi|^{2}}$ belongs to $L^{3 / 2, \infty}\left(Q^{\star}\right)$.

Let us now prove that $F_{n}(\xi, x, y)$ is bounded in $W^{1 / 2,1}\left(Q^{\star} \times Q^{2}\right)$. This will imply by the Sobolev embeddings theorem that $F_{n}(\xi, x, y)$ is relatively compact in $L^{p}\left(Q^{\star} \times Q^{2}\right)$, for all $1 \leqslant p<19 / 18$. In view of (5.20), Hölder's inequality yields the compactness in all $L^{p}\left(Q^{\star} \times Q^{2}\right)$, for all $1 \leqslant p<6$, and thus in particular the desired $L^{2}\left(Q^{\star} \times Q^{2}\right)$ compactness. To prove that $F_{n}(\xi, x, y)$ is bounded in $W^{1 / 2,1}\left(Q^{\star} \times Q^{2}\right)$, we prove that $F_{n}(\xi, x, y)$ is bounded both in $L^{1}\left(Q^{2} ; W^{1 / 2,1}\left(Q^{\star}\right)\right)$ and in $L^{1}\left(Q^{\star} ; W^{1 / 2,1}\left(Q^{2}\right)\right)$. The second bound is an immediate consequence of the fact that $F_{n}(\xi, x, y)$ is bounded in $L^{2}\left(Q^{\star} ; H^{1}\left(Q^{2}\right)\right)$. The latter fact holds because both $\rho_{n}(\xi, x, y)$ and $\nabla_{x} \rho_{n}(\xi, x, y)$ are bounded in $L^{2}\left(Q^{\star} \times Q^{2}\right), \frac{\mathrm{e}^{-i \xi \cdot(x-y)}}{|\xi|^{2}} \in L^{\infty}\left(Q^{2} ; L^{1}\left(Q^{\star}\right)\right)$, and

$$
\nabla_{x} F_{n}(\xi, x, y)=\nabla_{x} \rho_{n}^{*}(\xi, x, y) \star Q^{\star} \frac{\mathrm{e}^{-i \xi \cdot(x-y)}}{|\xi|^{2}}+\rho_{n}^{*}(\xi, x, y) \star Q^{\star} \frac{\nabla_{x} \mathrm{e}^{-i \xi \cdot(x-y)}}{|\xi|^{2}} .
$$

In order to show that $F_{n}(\xi, x, y)$ is bounded in $L^{1}\left(Q^{2} ; W^{1 / 2,1}\left(Q^{\star}\right)\right)$, we remark first that $\frac{1}{|\xi|^{2}} \in L^{\infty}\left(Q^{2}, W^{1 / 2,1}\left(Q^{\star}\right)\right.$, thus

$$
\begin{aligned}
\left\|F_{n}(\xi, x, y)\right\|_{W^{1 / 2,1}\left(Q^{\star}\right)} & =\left\|\rho_{n}(\xi, x, y) \star Q^{\star} \frac{\mathrm{e}^{-i \xi \cdot(x-y)}}{|\xi|^{2}}\right\|_{W^{1 / 2,1}\left(Q^{\star}\right)} \\
& \leqslant\left\|\rho_{n}(\xi, x, y)\right\|_{L^{1}\left(Q^{\star}\right)}\left\|\frac{1}{|\xi|^{2}}\right\|_{W^{1 / 2,1}\left(Q^{\star}\right)}
\end{aligned}
$$


whence we deduce

$$
\left\|F_{n}(\xi, x, y)\right\|_{L^{1}\left(Q^{2} ; W^{1 / 2,1}\left(Q^{\star}\right)\right)} \leqslant\left\|\rho_{n}(\xi, x, y)\right\|_{L^{1}\left(Q^{\star} \times Q^{2}\right)}\left\|\frac{1}{|\xi|^{2}}\right\|_{W^{1 / 2,1}\left(Q^{\star}\right)} .
$$

This concludes the first proof of (5.16), and thus the proof of Theorem 2.3.

Second proof of Theorem 2.3. - We may observe that we can modify the argument which is used in Step 3 above by proving the following.

LEMMA 5.2. - For any minimizing sequence $K_{n} \in \mathcal{K}$ of $I_{\mathrm{per}}^{H F}$, we have

$$
\lim _{n \rightarrow+\infty} \int_{Q} d x \int_{\mathbf{R}^{3}} \frac{\left|\rho_{n}(x, y)\right|^{2}}{|x-y|} d y=\int_{Q} d x \int_{\mathbf{R}^{3}} \frac{|\rho(x, y)|^{2}}{|x-y|} d y .
$$

Proof. - The proof of the above Lemma 5.1 yields in particular that the exchange term $\int_{Q} d x \int_{\mathbf{R}^{3}} \frac{\left|\rho_{n}(x, y)\right|^{2}}{|x-y|} d y$ is bounded independently of $n$, with the help of (5.13). Moreover, in view of (5.15), $\left|\rho_{n}(x, y)\right|^{2}$ converges to $|\rho(x, y)|^{2}$ almost everywhere on $\mathbf{R}^{3} \times \mathbf{R}^{3}$. Then, by Fatou's lemma, we deduce that

$$
\liminf _{n \rightarrow+\infty} \int_{Q} d x \int_{\mathbf{R}^{3}} \frac{\left|\rho_{n}(x, y)\right|^{2}}{|x-y|} d y \geqslant \int_{Q} d x \int_{\mathbf{R}^{3}} \frac{|\rho(x, y)|^{2}}{|x-y|} d y .
$$

In order to prove the reverse inequality for the upper limit, we argue as follows. Let $R>0$ be fixed, we may write

$$
\int_{Q} d x \int_{\mathbf{R}^{3}} \frac{\left|\rho_{n}(x, y)\right|^{2}}{|x-y|} d y=\int_{Q} d x \int_{|x-y| \leqslant R} \frac{\left|\rho_{n}(x, y)\right|^{2}}{|x-y|} d y+\int_{Q} d x \int_{|x-y| \geqslant R} \frac{\left|\rho_{n}(x, y)\right|^{2}}{|x-y|} d y .
$$

On the one hand, we obtain

$$
\begin{aligned}
\int_{Q} d x \int_{|x-y| \geqslant R} \frac{\left|\rho_{n}(x, y)\right|^{2}}{|x-y|} d y & \leqslant \frac{1}{R} \int_{Q} d x \int_{\mathbf{R}^{3}}\left|\rho_{n}(x, y)\right|^{2} d y \\
& =\frac{1}{R}\left\|\rho_{n}(\xi ; x, y)\right\|_{L^{2}\left(Q^{\star} \times Q \times Q\right)}^{2} \leqslant \frac{C}{R},
\end{aligned}
$$

for some positive constant $C$ that is independent of $n$, and because of the bound (5.13) on $\rho_{n}$.

On the other hand, we know from (5.15), that $\rho_{n}(x, y)$ converges to $\rho(x, y)$ strongly in $L_{\text {loc }}^{4}\left(\mathbf{R}^{3} \times \mathbf{R}^{3}\right)$. Thus, $\left|\rho_{n}(x, y)\right|^{2}$ converges to $|\rho(x, y)|^{2}$ strongly in $L_{\text {loc }}^{2}\left(\mathbf{R}^{3} \times \mathbf{R}^{3}\right)$. Since $\frac{1}{|x-y|} \chi_{|x-y| \leqslant R}(x, y)$ belongs to $L_{\text {loc }}^{2}\left(\mathbf{R}^{3} \times \mathbf{R}^{3}\right)$, we clearly obtain, for any fixed $R>0$,

$$
\lim _{n \rightarrow+\infty} \int_{Q} d x \int_{|x-y| \leqslant R} \frac{\left|\rho_{n}(x, y)\right|^{2}}{|x-y|} d y=\int_{Q} d x \int_{|x-y| \leqslant R} \frac{|\rho(x, y)|^{2}}{|x-y|} d y .
$$


Collecting (5.22) and (5.23), and letting $n$, and then $R$, go to infinity, we finally obtain

$$
\limsup _{n \rightarrow+\infty} \int_{Q} d x \int_{\mathbf{R}^{3}} \frac{\left|\rho_{n}(x, y)\right|^{2}}{|x-y|} d y \leqslant \int_{Q} d x \int_{\mathbf{R}^{3}} \frac{|\rho(x, y)|^{2}}{|x-y|} d y .
$$

The proof is now complete.

We shall now write down the system of Euler-Lagrange equations satisfied by any minimizer $K$ of $I_{\text {per }}^{H F}$. These equations look like very much the usual Hartree-Fock equations in Molecular Chemistry, and are likely to be the analogues in the periodic setting of the well-known Hartree-Fock equations. The form of these equations is very similar to the one we have derived for the periodic RHF model, namely (4.57)-(4.59), except for an extra term which comes from the exchange term. Arguing by analogy with the periodic RHF model, we obtain the following system of Euler-Lagrange equations, for almost every $\xi$ in $Q^{\star}$, and for every $n \geqslant 1$,

$$
\left\{\begin{array}{l}
-\Delta u_{n}(\xi, \cdot)-G u_{n}(\xi, \cdot) \\
\quad+\sum_{m \geqslant 1} \lambda_{m}(\xi)\left(\int_{Q} u_{m}(\xi, y)^{2} G(\cdot-y) d y\right) u_{n}(\xi, \cdot) \\
\quad-\iint_{Q^{\star} \times Q} \rho\left(\xi^{\prime} ; x ; y\right) W^{\infty}\left(\xi-\xi^{\prime} ; x-y\right) u_{n}(\xi, y) d y \frac{d \xi^{\prime}}{(2 \pi)^{3}} \\
=\sum_{m \geqslant 1} \varepsilon_{n m}(\xi) u_{m}(\xi, \cdot), \quad \text { a.e. on } Q, \\
\int_{Q}\left(\left|\nabla u_{n}(\xi, x)\right|^{2}-G(x)\left|u_{n}(\xi, x)\right|^{2}\right) d x \\
\quad+\sum_{m \geqslant 1} \lambda_{m}(\xi) D_{G}\left(\left|u_{n}(\xi, \cdot)\right|^{2},\left|u_{m}(\xi, \cdot)\right|^{2}\right) \\
\quad-\iiint_{Q^{\star} \times Q^{2}} \rho\left(\xi^{\prime} ; x ; y\right) W^{\infty}\left(\xi-\xi^{\prime} ; x-y\right) u_{n}^{*}(\xi, x) u_{n}(\xi, y) d x d y \frac{d \xi^{\prime}}{(2 \pi)^{3}} \\
=\mu_{n}^{0}(\xi)+\mu_{n}^{1}(\xi)+\pi,
\end{array}\right.
$$

with $\rho\left(\xi^{\prime} ; x ; y\right)=\sum_{m \geqslant 1} \lambda_{m}\left(\xi^{\prime}\right) u_{m}\left(\xi^{\prime}, x\right) u_{m}^{*}\left(\xi^{\prime}, y\right)$, and where the Lagrange multipliers $\pi, \mu_{n}^{0}(\xi), \mu_{n}^{1}(\xi), \varepsilon_{n m}(\xi)$ are respectively associated to the set of constraints

$$
\left\{\begin{array}{l}
\int_{Q^{\star}} \sum_{n \geqslant 1} \lambda_{n}(\xi) \frac{d \xi}{(2 \pi)^{3}}=1, \\
0 \leqslant \lambda_{n}(\xi) \leqslant 1, \text { for all } n \geqslant 1, \text { and for almost all } \xi \in Q^{\star}, \\
\int_{Q} u_{n}(\xi, x) u_{m}^{*}(\xi, x) d x=\delta_{n, m}, \text { for almost all } \xi \in Q^{\star} .
\end{array}\right.
$$


Since, once more, $E_{\mathrm{per}}^{H F}(K)$ is independent of the choice of an eigenbasis for $K_{\xi}$, we may assume without loss of generality that the matrix of $\varepsilon_{n m}(\xi)$ is diagonal, for almost every $\xi$ in $Q^{\star}$; under this assumption, the right-hand side term, $\sum_{m \geqslant 1} \varepsilon_{n m}(\xi) u_{m}(\xi, \cdot)$, in the first equation of (5.24) becomes simply $\varepsilon_{n}(\xi) u_{n}(\xi, \cdot)$. In addition, the Lagrange multipliers still satisfy the properties (4.58a), (4.58b), and (4.59), that we recall here for the convenience of the reader; namely, for all $n \geqslant 1$ and for almost every $\xi$ in $Q^{\star}$,

$$
\begin{aligned}
& \mu_{n}^{0}(\xi) \begin{cases}=0, & \text { if } \lambda_{n}(\xi)>0, \\
\geqslant 0, & \text { if } \lambda_{n}(\xi)=0,\end{cases} \\
& \mu_{n}^{1}(\xi) \begin{cases}=0, & \text { if } \lambda_{n}(\xi)<1, \\
\leqslant 0, & \text { if } \lambda_{n}(\xi)=1,\end{cases}
\end{aligned}
$$

and

$$
\left\{\begin{array}{l}
\lambda_{n}(\xi)=0 \quad \Longrightarrow \varepsilon_{n}(\xi) \geqslant \pi, \\
0<\lambda_{n}(\xi)<1 \Longrightarrow \varepsilon_{n}(\xi)=\pi, \\
\lambda_{n}(\xi)=1 \quad \Longrightarrow \varepsilon_{n}(\xi) \leqslant \pi .
\end{array}\right.
$$

Once we have established the existence of a minimizer for the periodic Hartree-Fock model, we are able to compare from above the Hartree-Fock energy per unit volume with this periodic problem.

\subsection{Upper limit of the energy per unit volume}

In this section, we prove the following.

Proposition 2.1. - We assume that the Van Hove sequence $\Lambda$ satisfies (2.21). In addition, we assume that the unit cell $Q$ is a cube, and that there exists a minimizer $K \in \mathcal{K}$ of $I_{\mathrm{per}}^{H F}$ whose density $\rho$ shares the symmetries of the unit cube. Then,

$$
\limsup _{\Lambda \rightarrow \infty} \frac{I_{\Lambda}^{H F}}{|\Lambda|} \leqslant I_{\text {per }}^{H F}+\frac{M}{2},
$$

where $I_{\mathrm{per}}^{H F}$ is defined by (2.23)-(2.24).

Remark 5.1.-

(1) The same result holds true in the smeared nuclei case, if we assume moreover that $m$ shares the symmetries of the cube $Q$, and define $M$ according to (2.22).

(2) In the HF setting, since we do not know whether $\rho$ is unique, the assertion that $\rho$ shares the symmetries of the cube needs to be assumed.

Proof of Proposition 2.1. - The beginning of the proof is the same as in the proof of Proposition 4.2 for the RHF problem. Let us denote by $K$ a minimizer of the periodic HF problem such that the corresponding density $\rho$ shares the symmetries of the unit cube. We set

$$
\partial \Lambda=\{k \in \Lambda ; d(k, \partial \Gamma(\Lambda)) \leqslant 2\},
$$




$$
\begin{gathered}
\Gamma(\partial \Lambda)=\bigcup_{k \in \partial \Lambda} k+Q, \\
\stackrel{\circ}{\Lambda}=\Lambda \backslash \partial \Lambda,
\end{gathered}
$$

and

$$
\Gamma(\stackrel{\circ}{\Lambda})=\bigcup_{\substack{\vdots \\ k \in \Lambda}} k+Q=\Gamma(\Lambda) \backslash \Gamma(\partial \Lambda) .
$$

We build a cut-off function $\chi_{\Lambda} \in \mathcal{D}\left(\mathbf{R}^{3}\right)$ satisfying the following properties: $0 \leqslant \chi_{\Lambda} \leqslant 1$, $\chi_{\Lambda} \equiv 1$ on $\Gamma(\stackrel{\circ}{\Lambda}), \chi_{\Lambda} \equiv 0$ on $\Gamma(\Lambda)^{c}$. In particular, we have

$$
\int_{\mathbf{R}^{3}} \chi_{\Lambda}^{2}(x) \rho(x, x) d x=|\Lambda|+\mathrm{o}(|\Lambda|) \leqslant|\Lambda| .
$$

We next consider the operator $K_{\Lambda}$ on $L^{2}\left(\mathbf{R}^{3}\right)$ whose kernel is

$$
\rho_{\Lambda}(x, y)=\chi_{\Lambda}(x) \rho(x, y) \chi_{\Lambda}(y) .
$$

Then, because of (5.26), and since $I_{\Lambda}^{H F}$ is also obtained by minimizing $E_{\Lambda}^{H F}$ when the trace constraint is relaxed, that is on the set of self-adjoint operators

$$
\mathcal{K}_{\Lambda}^{\prime}=\left\{0 \leqslant K \leqslant \mathbf{1}, \operatorname{Tr} K \leqslant|\Lambda|, \operatorname{Tr}\left[\left(-\Delta-V_{\Lambda}\right) K\right]<+\infty\right\},
$$

we have

$$
\frac{I_{\Lambda}^{H F}}{|\Lambda|} \leqslant \frac{1}{|\Lambda|} E_{\Lambda}^{H F}\left(K_{\Lambda}\right)
$$

We show exactly as in the proof of Proposition 4.2 that

$$
\begin{gathered}
\lim _{\Lambda \rightarrow \infty} \frac{1}{|\Lambda|}\left[\operatorname{Tr}_{L^{2}\left(\mathbf{R}^{3}\right)}\left[-\Delta K_{\Lambda}\right]-\int_{\mathbf{R}^{3}} V_{\Lambda} \rho_{\Lambda}+\frac{1}{2} D\left(\rho_{\Lambda}, \rho_{\Lambda}\right)+\frac{1}{2} U_{\Lambda}\right] \\
=\int_{Q^{\star}} \operatorname{Tr}_{L_{\xi}^{2}(Q)}\left[-\Delta_{\xi} K_{\xi}\right] \frac{d \xi}{(2 \pi)^{3}}-\int_{Q} G \rho+\frac{1}{2} D_{G}(\rho, \rho)+\frac{M}{2} .
\end{gathered}
$$

According to the definition (2.5) of the Hartree-Fock energy, to reach the conclusion, it now remains to compute the exchange term corresponding to $K_{\Lambda}$, and to show that

$$
\lim _{\Lambda \rightarrow \infty} \frac{1}{|\Lambda|} \iint_{\mathbf{R}^{3} \times \mathbf{R}^{3}} \frac{\left|\rho_{\Lambda}(x, y)\right|^{2}}{|x-y|} d x d y=\int_{Q} d x \int_{\mathbf{R}^{3}} \frac{|\rho(x, y)|^{2}}{|x-y|} d y .
$$

It is easily seen that

$$
\frac{1}{|\Lambda|} \iint_{\mathbf{R}^{3} \times \mathbf{R}^{3}} \frac{\left|\rho_{\Lambda}(x, y)\right|^{2}}{|x-y|} d x d y
$$




$$
\begin{aligned}
= & \frac{1}{|\Lambda|} \iint_{\mathbf{R}^{3} \times \mathbf{R}^{3}} \frac{\chi_{\Lambda}(x)^{2}|\rho(x, y)|^{2} \chi_{\Lambda}(y)^{2}}{|x-y|} d x d y \\
= & \frac{1}{|\Lambda|} \iint_{\substack{\Gamma(\Lambda) \times \Gamma(\Lambda) \\
(x)}} \frac{|\rho(x, y)|^{2}}{|x-y|} d x d y+\frac{2}{|\Lambda|} \iint_{\Gamma(\Lambda) \times \Gamma(\partial \Lambda)} \frac{\chi_{\Lambda}(x)^{2}|\rho(x, y)|^{2}}{|x-y|} d x d y \\
& +\frac{1}{|\Lambda|} \int_{\Gamma(\partial \Lambda) \times \Gamma(\partial \Lambda)} \frac{\chi_{\Lambda}(x)^{2}|\rho(x, y)|^{2} \chi_{\Lambda}(y)^{2}}{|x-y|} d x d y .
\end{aligned}
$$

Let us first concentrate on the last two terms and show that they converge to 0 as $\Lambda$ goes to infinity. Indeed, since $0 \leqslant \chi_{\Lambda} \leqslant 1$, the sum of these two terms is a fortiori less or equal to the following quantity

$$
Q_{\Lambda}=\frac{2}{|\Lambda|} \iint_{\mathbf{R}^{3} \times \Gamma(\partial \Lambda)} \frac{|\rho(x, y)|^{2}}{|x-y|} d x d y
$$

which makes sense since

$$
\int_{Q} d x \int_{\mathbf{R}^{3}} \frac{|\rho(x, y)|^{2}}{|x-y|} d y<+\infty .
$$

Besides, using the fact that $\rho(x+k, y+k)=\rho(x, y)$, for every $k$ in $\mathbf{Z}^{3}$, we have

$$
\begin{aligned}
Q_{\Lambda} & =\frac{2}{|\Lambda|} \sum_{k \in \partial \Lambda} \int_{Q} d x \int_{\mathbf{R}^{3}} \frac{|\rho(x+k, y)|^{2}}{|x+k-y|} d y \\
& =\frac{2}{|\Lambda|} \sum_{k \in \partial \Lambda} \int_{Q} d x \int_{\mathbf{R}^{3}} \frac{|\rho(x, y-k)|^{2}}{|x-(y-k)|} d y \\
& =2 \frac{|\partial \Lambda|}{|\Lambda|} \int_{Q} d x \int_{\mathbf{R}^{3}} \frac{|\rho(x, y)|^{2}}{|x-y|} d y .
\end{aligned}
$$

We conclude easily, since by definition of Van Hove sequences, $|\partial \Lambda|=\mathrm{o}(|\Lambda|)$, as $\Lambda$ goes to infinity. Therefore, it remains to check that

$$
\frac{1}{|\Lambda|} \int_{\Gamma(\Lambda) \times \Gamma(\Lambda)} \frac{|\rho(x, y)|^{2}}{|x-y|} d x d y=\int_{Q} d x \int_{\mathbf{R}^{3}} \frac{|\rho(x, y)|^{2}}{|x-y|} d y+\mathrm{o}(1),
$$

since it is clear that, for the same reasons as above,

$$
\frac{1}{|\Lambda|} \iint_{\Gamma(\Lambda) \times \Gamma(\Lambda)} \frac{|\rho(x, y)|^{2}}{|x-y|} d x d y=\frac{1}{|\Lambda|} \iint_{\substack{0 \\ \Gamma(\Lambda) \times \Gamma(\Lambda)}} \frac{|\rho(x, y)|^{2}}{|x-y|} d x d y+\mathrm{o}(1) .
$$

We rewrite this sum in the following way 


$$
\begin{aligned}
\frac{1}{|\Lambda|} \iint_{\Gamma(\Lambda) \times \Gamma(\Lambda)} \frac{|\rho(x, y)|^{2}}{|x-y|} d x d y & =\frac{1}{|\Lambda|} \sum_{k, l \in \Lambda} \iint_{Q \times Q} \frac{|\rho(x+k, y+l)|^{2}}{|x+k-y-l|} d x d y \\
& =\frac{1}{|\Lambda|} \sum_{k, l \in \Lambda} \iint_{Q \times Q} \frac{|\rho(x, y+l-k)|^{2}}{|x-(y+l-k)|} d x d y \\
& =\frac{1}{|\Lambda|} \sum_{k, l \in \Lambda} \iint_{Q \times \mathbf{R}^{3}} \chi_{l-k+Q}(y) \frac{|\rho(x, y)|^{2}}{|x-y|} d x d y .
\end{aligned}
$$

The conclusion is then easily reached by showing that the sequence of functions defined by $\frac{1}{|\Lambda|} \sum_{k, l \in \Lambda} \chi_{l-k+Q}(y)$ converges to 1 in $L^{\infty}\left(\mathbf{R}^{3}\right) \star$-weak, and almost everywhere on $\mathbf{R}^{3}$. To prove this claim, we could directly apply the technical lemma given in Chapter 2 of [11], but, since the proof is simple, we reproduce the argument here. On the one hand, we clearly have

$$
0 \leqslant \frac{1}{|\Lambda|} \sum_{k, l \in \Lambda} \chi_{l-k+Q}(y) \leqslant \frac{1}{|\Lambda|} \sum_{k \in \Lambda} \sum_{l \in \mathbf{Z}^{3}} \chi_{l-k+Q}(y) \leqslant 1 .
$$

Therefore, the sequence is bounded in $L^{\infty}\left(\mathbf{R}^{3}\right)$. On the other hand, let $y$ be fixed in $\mathbf{R}^{3}$. Then, there exists $m \in \mathbf{Z}^{3}$ such that $y$ lies in $m+Q$, and for $\Lambda$ large enough $m$ lies in $\Lambda-\Lambda$, by definition of a Van Hove sequence. Hence, for such an $y$, we obtain

$$
\begin{aligned}
1 & =\frac{1}{|\Lambda|} \sum_{k \in \Lambda} \sum_{l \in \mathbf{Z}^{3}} \chi_{l-k+Q}(y)=\frac{1}{|\Lambda|} \#\{k, l \in \Lambda ; l-k=m\} \\
& =\frac{1}{|\Lambda|} \#\{k \in \Lambda ; k-m \in \Lambda\}=\frac{1}{|\Lambda|} \#[\Lambda \cap(\Lambda+m)] \\
& \geqslant \frac{1}{|\Lambda|} \#\left[\Lambda \backslash \Lambda^{|m|+\frac{1}{2}}\right] \geqslant 1-\frac{\mid \Lambda^{|m|+\frac{1}{2} \mid}}{|\Lambda|},
\end{aligned}
$$

where $\Lambda^{|m|+\frac{1}{2}}=\left\{k \in \Lambda ; d(k ; \partial \Gamma(\Lambda)) \leqslant|m|+\frac{1}{2}\right\}$, and where the notation \#S stands for the number of elements in the set $S$. We conclude easily since, by definition of a Van Hove sequence, $\frac{\mid \Lambda^{|m|+\frac{1}{2} \mid}}{|\Lambda|}$ goes to 0 as $\Lambda$ goes to infinity.

\section{Extensions and perspectives}

We list in this last section a few comments on the above results, and indicate some possible extensions of our work.

So far, we have assumed that the periodic lattice that is covered in the limit by the sequence $\Lambda$ is $\mathbf{Z}^{3}$, and thus that the periodic cell $Q$ is a cube of unit size. The first basic observation to make is that our whole work goes through mutatis mutandis if we replace the cube of unit size by a cube of size $R$. Slight modifications must be made in the definition of the potential $G$ in particular, and we refer the reader to [11] for such modifications. 
Replacing the cube by another shape of unit cell is another story. As we have mentioned above, Theorems 2.1 and 2.3 still hold. On the contrary, our strategy of proof for Theorem 2.2 depends upon the shape of the cell. It is an open (but rather technical) question to extend this result to other shapes of cells.

Likewise, we have mentioned above that the assumption (2.21) is a technical assumption required only for the proof of Theorem 2.2. We recall we believe it can be left apart, but we do not know how.

Concerning the periodic problems per se, it would be an interesting question to address to see whether one can say something on the minimizers. In the HF setting, for instance, we are not able to check, for the time being, whether or not the minimizing periodic density matrix $K$ is a projector (which is equivalent to the fact that $K_{\xi}$ is a (finite rank) projector, for almost all $\xi$ in $Q^{\star}$ ), as it is the case for the Hartree-Fock model for molecules.

Apart from these somewhat secondary questions, the main issue to tackle is the proof of the thermodynamic limit for the energy per unit volume in the $\mathrm{H}$ and HF settings. As far as this question is concerned, much remains to be done.

Even in some simplified framework, trying to understand Hartree-Fock type models for quasicrystals would also be of interest. Our study [11] and references $[1,3,8,47]$ could constitute a starting point.

Finally, let us mention that the periodic problems we have defined in this work can be treated numerically, and we indeed intend to treat them numerically. Numerical experiment might in particular give some insight into the mathematical nature of these models and help oneself to make up his mind on some of the questions mentioned above.

\section{Acknowledgement}

The authors would like to thank V. Bach for several stimulating discussions, and J.P. Solovej for valuable comments on a preliminary version of the manuscript.

\section{REFERENCES}

[1] Amerio L., Prouse G., Almost Periodic Functions and Functional Equations, Van Nostrand Reinhold Company, 1971.

[2] Ashcroft N.W., Mermin N.D., Solid-state Physics, Saunders College Publishing, 1976.

[3] Axel F., Gratias D. (Eds.), Beyond Quasicrystals, Centre de Physique Les Houches, Les Editions de Physique, Springer, 1995.

[4] Bach V., Lieb E.H., Solovej J.P., Generalized Hartree-Fock theory and the Hubbard model, J. Stat. Phys. 76 (1994) 3-90.

[5] Bach V., Error bound for the Hartree-Fock energy of atoms and molecules, Comm. Math. Phys. 147 (1992) 527-548.

[6] Balian R., From Microphysics to Macrophysics; Methods and Applications of Statistical Physics, I, II, Springer-Verlag, 1991.

[7] Bloch F., Über die Quantenmechanik der Electronen in Kristallgittern, Z. Phys. 52 (1928) 555-560.

[8] Bohr H., Almost Periodic Functions, Chelsea, 1947.

[9] Callaway J., Quantum Theory of the Solid State, Academic Press, 1974. 
[10] Catto I., Le Bris C., Lions P.-L., Limite thermodynamique pour des modèles de type Thomas-Fermi [Thermodynamic limit for Thomas-Fermi type models], C. R. Acad. Sci. Paris Sér. I Math. 322 (1996) 357-364.

[11] Catto I., Le Bris C., Lions P.-L., Mathematical Theory of Thermodynamic Limits: ThomasFermi Type Models, Oxford University Press, 1998.

[12] Catto I., Le Bris C., Lions P.-L., Sur la limite thermodynamique pour des modèles de type Hartree et Hartree-Fock [On the thermodynamic limit for Hartree and Hartree-Fock type models], C. R. Acad. Sci. Paris Sér. I Math. 327 (1998) 259-266.

[13] Catto I., Le Bris C., Lions P.-L., On some periodic Hartree-type models for crystals, submitted. Also available at: http://www.math.utexas.edu/mp_arc/c/99/99-392.ps.gz.

[14] Chen L., Moody R.V., Patera J., Non-crystallographic root systems, in: Quasicrystals and Discrete Geometry (Toronto, ON, 1995), Fields Institute Monogr., Vol. 10, American mathematical society, Providence, RI, 1998, pp. 135-178.

[15] Conca C., Vanninathan M., Homogenization of periodic structures via Bloch decomposition, SIAM J. Appl. Math. 57 (6) (1997) 1639-1659.

[16] Conca C., Planchard J., Vanninathan M., Fluids and Periodic Structures, Collection RAM, Vol. 38, Wiley/Masson, Paris, 1995.

[17] Eastham M.S.P., The Spectral Theory of Periodic Differential Equations, Scottish Acad. Press, Edinburgh-London, 1973.

[18] Figotin A., Kuchment P., Band-gap structure of spectra of periodic dielectric and acoustic media. I. Scalar model, SIAM J. Appl. Math. 56 (1) (1996) 68-88.

[19] Figotin A., Kuchment P., Band-gap structure of spectra of periodic dielectric and acoustic media. II. Two-dimensional photonic crystals, SIAM J. Appl. Math. 56 (6) (1996) 15611620.

[20] Floquet G., Sur les équations différentielles linéaires à coefficients périodiques, Ann. Ecole Norm. Sér. 212 (1883) 47-89.

[21] Friesecke G., Pair correlations and exchange phenomena in the free electron gas, Comm. Math. Phys. 184 (1997) 143-171.

[22] Karpeshina Y.E., Perturbation theory for the Schrödinger operator with a periodic potential, Lecture Notes in Mathematics, Vol. 1663, Springer-Verlag, 1997.

[23] Kittel C., Introduction to Solid State Physics, 6th edn., Wiley, 1986.

[24] Kuchment P., Floquet Theory for Partial Differential Equations, Operator Theory Advances and Applications, Vol. 60, Birkhäuser, Basel, 1993.

[25] Lebowitz J.L., Lieb E.H., Existence of thermodynamics for real matter with Coulomb forces, Phys. Rev. Lett. 22 (13) (1969) 631-634.

[26] Lieb E.H., Lebowitz J.L., The constitution of matter: existence of thermodynamics for systems composed of electrons and nuclei, Adv. in Maths. 9 (1972) 316-398.

[27] Lieb E.H., Lebowitz J.L., Lectures on the thermodynamic limit for Coulomb systems, in: Springer Lecture Notes in Physics, Vol. 20, Springer, 1973, pp. 136-161.

[28] Lieb E.H., The stability of matter: from atoms to stars, Bull. Amer. Math. Soc. 22 (1) (1990) $1-49$.

[29] Lieb E.H., Thomas-Fermi and related theories of atoms and molecules, Rev. Modern Phys. 53 (4) (1981) 603-641; Errata: Rev. Modern Phys. 54 (1982) 311.

[30] Lieb E.H., A Variational principle for many-fermion systems, Phys. Rev. Lett. 46 (1981) 457-459; Errata: Rev. Modern Phys. 47 (1981) 69.

[31] Lieb E.H., Oxford S., An improved lower bound on the indirect Coulomb energy, Int. J. Quantum Chem. 19 (1981) 427-439.

[32] Lieb E.H., Simon B., The Thomas-Fermi theory of atoms, molecules and solids, Adv. Math. 23 (1977) 22-116. 
[33] Lieb E.H., Simon B., The Hartree-Fock theory for Coulomb systems, Comm. Math. Phys. 53 (1977) 185-194.

[34] Lieb E.H., Solovej J.P., Yngvason J., Asymptotics of heavy atoms in high magnetic fields: I. Lowest Landau band regions, Comm. Pure. Appl. Math. 47 (4) (1994) 513-591.

[35] Lieb E.H., Thirring W., Inequalities for the moments of the eigenvalues of the Schrödinger Hamiltonian and their relation to Sobolev inequalities, in: Lieb E.H., Simon B., Wightman A. (Eds.), Studies in Mathematical Physics, Princeton University Press, 1976, pp. 269-303.

[36] Lieb E.H., Thirring W., Bounds for the kinetic energy of fermions which prove the stability of matter, Phys. Rev. Lett. 35 (1975) 687-689; Errata: Phys. Rev. Lett. 35 (1975) 1116.

[37] Lions P.-L., Solutions of Hartree-Fock equations for Coulomb systems, Comm. Math. Phys. 109 (1987) 33-97.

[38] Lions P.-L., Hartree-Fock and related equations, in: Nonlinear Partial Differential Equations and their Applications, Lect. Collège de France Seminar, Vol. IX, Paris, 1985-86, Pitman Res. Notes Math. Ser., Vol. 181, 1988, pp. 304-333.

[39] Lions P.-L., Paul T., Sur les mesures de Wigner, Rev. Mat. Iberoamericana 9 (3) (1993) 553-618.

[40] Madelung O., Introduction to Solid State theory, Solid State Sciences, Vol. 2, SpringerVerlag, Berlin, 1981.

[41] Parr R.G., Yang W., Density-Functional Theory of Atoms and Molecules, Oxford University Press, Oxford, 1989.

[42] Pisani C., in: Quantum Mechanical Ab Initio Calculation of the Properties of Crystalline Materials, Lecture Notes in Chemistry, Vol. 67, Springer-Verlag, 1996.

[43] Quinn Ch.M., An introduction to the Quantum Theory of Solids, Clarendon Press, Oxford, 1973.

[44] Reed M., Simon B., Methods of Modern Mathematical Physics, I: Functional Analysis, Academic Press, New-York-London, 1972.

[45] Reed M., Simon B., Methods of Modern Mathematical Physics, IV: Analysis of Operators, Academic Press, New-York, 1978.

[46] Ruelle D., Statistical Mechanics: Rigorous Results, Benjamin, New-York, 1969; Advanced Books Classics, Addison-Wesley, 1989.

[47] Senechal M., Quasicrystals and Geometry, Cambridge University Press, 1995.

[48] Slater J.C., Quantum Theory of Molecules and Solids, Mac Graw Hill, 1963.

[49] Slater J.C., Symmetry and Energy Bands in Crystals, Dover, 1972.

[50] Solovej J.P., Universality in the Thomas-Fermi-von Weizsäcker model of atoms and molecules, Comm. Math. Phys. 129 (1990) 561-598.

[51] Solovej J.P., An improvement on stability of matter in mean field theory, in: Differential Equations and Mathematical Physics, Proceedings of the International Conference, Univ. of Alabama, Birmingham, March 1994, International Press, 1995.

[52] Solovej J.P., Proof of the ionization conjecture in a reduced Hartree-Fock model, Invent. Math. 104 (1991) 291-311.

[53] Stein E., Singular Integrals Operators and Differentiability of Functions, Princeton University Press, Princeton, 1970.

[54] Temam R., Infinite-Dimensional Dynamical Systems in Mechanics and Physics, SpringerVerlag, 1988.

[55] Tolman R.C., The Principles of Statistical Mechanics, Oxford University Press, 1962.

[56] Wilcox C., Theory of Bloch waves, J. Anal. Math. 33 (1978) 146-167.

[57] Ziman J., Principles of the Theory of Solids, 2nd edn., Cambridge University Press, 1972. 